Aus der Abteilung Mund - , Kiefer - und Gesichtschirurgie

(Prof. Dr. med. Dr. med. dent. H. Schliephake)

im Zentrum Zahn - , Mund - und Kieferheilkunde

der Medizinischen Fakultät der Universität Göttingen

\title{
Struktur und Dimension des oropharyngealen Luftweges im Digitalen Volumentomographen (DVT)
}

\author{
INAUGURAL - DISSERTATION \\ zur Erlangung des Doktorgrades \\ für Zahnheilkunde \\ der Medizinischen Fakultät \\ der Georg - August - Universität zu Göttingen \\ vorgelegt von \\ Christoph Schlick \\ aus \\ der Lutherstadt Wittenberg
}

Göttingen 2011 
D e k a n:

I. Berichterstatter:

II. Berichterstatter/in:

III. Berichterstatter/in:

Tag der mündlichen Prüfung:
Prof. Dr. rer. nat. H. Kroemer

Prof. Dr. med. Dr. med. dent. W. Engelke

PD Dr. Arno Olthoff

Prof. Dr. Martin Oppermann

07.10.2014 


\section{Inhaltsverzeichnis}

$\begin{array}{ll}\text { 1. Einleitung } & 1\end{array}$

$\begin{array}{ll}\text { 1.1. Einführung in das Thema } & 1\end{array}$

1.2. Anatomie des orofazialen Systems 2

1.2.1. Systematische Anatomie 2

1.2.1.1. Pharyngeale Strukturen 2

1.2.1.2. Orale Strukturen 4

1.2.2. Funktionelle Anatomie nach dem Funktionskompartimentmodell 7

1.2.3. Röntgenologische Anatomie 9

1.3. Bildgebende Verfahren zur Darstellung des oberen Luftweges 11

1.3.1. Zweidimensionale Aufnahmen mittels lateralem Fernröntgenseitenbild 11

1.3.2. Computertomographie (CT) 12

1.3.3. Digitale Volumentomographie (DVT) 14

1.3.4. Magnetresonanztomographie (MRT) 16

$\begin{array}{ll}\text { 1.4. Ziel der Arbeit } & 17\end{array}$

$\begin{array}{ll}\text { 2. Material und Methode } & 18\end{array}$

$\begin{array}{ll}\text { 2.1. Patientenkollektiv } & 18\end{array}$

2.2. Digitaler Volumentomograph NewTom QR-9000@ 18

$\begin{array}{ll}\text { 2.3. Konstanzprüfung des DVT } & 20\end{array}$

$\begin{array}{ll}\text { 2.4. DVT-Softwareeinstellungen } & 21\end{array}$

$\begin{array}{ll}\text { 2.5. Vermessung der DVT-Datensätze } & 21\end{array}$

$\begin{array}{ll}\text { 2.6. Statistische Auswertung } & 27\end{array}$

$\begin{array}{ll}\text { 2.7. Ethikkommissionsvotum } & 27\end{array}$ 
$\begin{array}{lr}\text { 3. Ergebnisse } & 28\end{array}$

3.1. Luftweg und benachbarte Strukturen im Geschlechtervergleich 28

3.1.1. Mediosagittale Messungen 28

3.1.2. Messungen unter Bezug auf axiale Schichten 33

3.1.3. Koronale Messungen 43

3.2. Korrelation der Messwerte bei Männern 45

3.2.1. Korrelation des kollapsfähigen Segmentes mit ausgewählten Parametern 45

3.2.2. Korrelation der Luftwegsquerschnitte auf unterschiedlichen anatomischen Ebenen 45

3.2.3. Korrelation der sagittalen und transversalen Dimensionen des Luftweges 46

3.2.4. Neigung des Unterkieferastes zur mediosagittalen Ebene und Korrelation zum

$\begin{array}{ll}\text { Luftwegsquerschnitt } & 47\end{array}$

3.3. Korrelation der Messwerte bei Frauen $\quad 48$

3.3.1. Korrelation des kollapsfähigen Segmentes mit ausgewählten Parametern 48

3.3.2. Korrelation der Luftwegsquerschnitte auf unterschiedlichen anatomischen Ebenen 48

3.3.3. Korrelation der sagittalen und transversalen Dimensionen des Luftweges 49

3.3.4. Neigung des Unterkieferastes zur mediosagittalen Ebene und Korrelation zum

$\begin{array}{ll}\text { Luftwegsquerschnitt } & 50\end{array}$

$\begin{array}{ll}\text { 4. Diskussion } & 51\end{array}$

4.1. Kritische Betrachtung der angewandten Methode 52

4.1.1. Aspekte der Patientenauswahl 52

4.1.2. Aufnahmebedingungen 53

4.1.3. Technische Aspekte der Digitalen Volumentomographie 54

4.1.4. Auswahl der Messparameter 56

4.2. Anatomischer Vergleich zwischen den Geschlechtern $\quad 57$

4.3. Vergleich der Ergebnisse mit anderen Studien $\quad 61$

4.3.1. Messergebnisse im mediosagittalen Schnitt 61

4.3.2. Messungen unter Bezug auf axiale Schichten 63

4.3.3. Messergebnisse im koronalen Schnitt 66 
6. Abkürzungsverzeichnis

7.1. Fallbeispiele 75

7.1.1. Schnitt in der mediosagittalen Ebene 75

7.1.2. Axialer Schnitt auf Höhe des unteren Inzisialpunktes (MPW) 75

7.1.3. Koronaler Schnitt auf der Hälfte der Nasallinie 76

8. Literaturverzeichnis 


\section{Abbildungsverzeichnis:}

Abb. 1: Sagittales Schema mit Einteilung des Pharynx und angrenzenden Strukturen entsprechend der anatomischen Lokalisation

Abb. 2: Funktionseinheiten des oronasopharyngealen Systems.

Abb. 3: Funktionskompartimente des oronasopharyngealen Systems.

Abb. 4: Schematische Darstellung von Hart- und Weichgewebspunkten.

Abb. 5: Funktionsprinzip der Spiral-Computertomographie

Abb. 6: Funktionsprinzip der Digitalen Volumentomographie (DVT).

Abb. 7: Prüfkörper für die Konstanzprüfung des NewTom QR-DVT 9000@

Abb. 8: Prüfkörper für die Konstanzprüfung des NewTom QR-DVT 9000@

Abb. 9: Schnitt in der Mediosagittalen (modifiziert nach Markiewicz 2011)

Abb. 10: Schnitt in der Mediosagittalen (nach Markiewicz 2011)

Abb. 11: Koronaler Schnitt auf der Hälfte der Nasallinie (nach Markiewicz 2011)

Abb. 12: Axialer Schnitt auf Höhe des unteren Inzisialpunktes (MPW) (nach Markiewicz 2011)

Abb. 13: Box-Plots und Histogramme für die Strecke ANS-PNS

Abb. 14: Box-Plots und Histogramme für die Weichgaumenlänge

Abb. 15: Box-Plots und Histogramme für die Weichgaumenbreite

Abb. 16: Box-Plots und Histogramme für die Strecke PNS-Epiglottis

Abb. 17: Box-Plots und Histogramme für die Strecke Hyoid zur Nasallinie

Abb. 18: Box-Plots und Histogramme für die Strecke ANS-Hyoidsenkrechte

Abb. 19: Box-Plots und Histogramme für die Kontaktstrecke Zunge und weicher Gaumen

Abb. 20: Box-Plots und Histogramme für den subpalatinalen Luftraum

Abb. 21: Box-Plots und Histogramme für die Strecke Isthmus-Nasallinie

Abb. 22: Box-Plots und Histogramme für die Strecke UPW anterior-posterior

Abb. 23: Box-Plots und Histogramme für die Strecke UPW transversal

Abb. 24: Box-Plots und Histogramme für die Fläche UPW

Abb. 25: Box-Plots und Histogramme für die Strecke Isthmus anterior-posterior

Abb. 26: Box-Plots und Histogramme für die Strecke Isthmus transversal

Abb. 27: Box-Plots und Histogramme für die Fläche Isthmus

Abb. 28: Box-Plots und Histogramme für die Strecke Isthmus axial

Abb. 29: Box-Plots und Histogramme für die Strecke MPW anterior-posterior

Abb. 30: Box-Plots und Histogramme für für die Strecke MPW transversal

Abb. 31: Box-Plots und Histogramme für die Fläche MPW 
Abb. 33: Box-Plots und Histogramme für die Strecke TPW transversal

Abb. 34: Box-Plots und Histogramme für die Fläche TPW

Abb. 35: Box-Plots und Histogramme für die Strecke LPW anterior-posterior

Abb. 36: Box-Plots und Histogramme für die Strecke LPW transversal

Abb. 37: Box-Plots und Histogramme für die Fläche LPW

Abb. 38: Box-Plots und Histogramme für den Winkel $\alpha$

Abb. 39: Box-Plots und Histogramme für den Winkel $\beta$

Abb. 40: Box-Plots und Histogramme für die intermaxilläre Raumlänge

Abb. 41: Box-Plots und Histogramme für die Strecke Nasallinie-Unterkiefer

Abb. 42: Box-Plots und Histogramme für den Abstand der lingualen Höcker

Abb. 43: Box-Plots und Histogramme für die palatinale Höhe

Abb. 44: Mediosagittale Schnitte

Abb. 45: Schnitt auf Höhe der Inzisialkante

Abb. 46: Koronaler Schnitt auf der Hälfte der Nasallinie 


\section{Tabellenverzeichnis:}

Tab. 1: Überblick über kephalometrische Messwerte. $\quad 11$

Tab. 2: Auflistung der Messpunkte. $\quad 22$

Tab. 3: Auflistung der Messstrecken. $\quad 22$

Tab. 4: Korrelation der Strecke PNS-Epiglottis mit ausgewählten Parametern für Männer.

Tab. 5: Korrelation der Querschnitte des Luftweges für Männer.

Tab. 6: Korrelation der sagittalen und transversalen Dimensionen des Luftweges für Männer. 46

Tab. 7: Korrelation der Winkel innen und außen mit dem Luftweg auf Höhe MPW für Männer. $\quad 47$

Tab. 8: Korrelation der Strecke PNS-Epiglottis mit ausgewählten Parametern für Frauen. $\quad \mathbf{4 8}$

Tab. 9: Korrelation der Querschnitte des Luftweges für Frauen. $\quad \mathbf{4 8}$

Tab. 10: Korrelation der sagittalen und transversalen Dimensionen des Luftweges für Frauen. 49

Tab. 11: Korrelation der Winkel $\alpha$ und $\beta$ mit dem Luftweg auf Höhe MPW für Frauen. $\quad 50$ 


\section{Einleitung}

\subsection{Einführung in das Thema}

Schlafbezogene Atemregulationsstörungen haben in vergangenen Jahren zunehmend an Bedeutung gewonnen. Durch zahlreiche Forschungsarbeiten wurde ersichtlich, dass es sich hierbei um sehr komplexe Krankheitsbilder handelt, deren mögliche Auswirkungen für den Allgemeinorganismus schwerwiegend sein können. Das steigende Interesse an diesen Erkrankungen hatte zur Folge, dass der Erfassung der Anatomie der oberen Luftwege besondere Aufmerksamkeit zuteil wurde. In früheren Studien wurde das orofaziale System mit verschiedenen Methoden, wie laterales Fernröntgenseitenbild, Computertomographie (CT) oder Magnetresonanztomographie (MRT) dargestellt, um folgende Ziele zu erreichen: Unterschiede in der Anatomie der oberen Atemwege aufdecken, Ätiologie und Pathophysiologie der Erkrankung klären sowie Patientenbetreuung und Behandlungserfolg verbessern.

Der Gebrauch des digitalen Volumentomographen in der zahnärztlichen Praxis wird weiter an Bedeutung gewinnen. Die dreidimensionale Darstellung des Gesichtsschädels vereinfacht die präimplantologische Planung und ermöglicht eine Risikoeinschätzung einer Nervschädigung bei Zahnextraktionen. Im Gegensatz zur Magnetresonanztomographie (MRT) und Computertomographie (CT) darf die Digitale Volumentomographie (DVT) nach entsprechender Fortbildung auch von einem Zahnarzt betrieben werden.

Ziele der Studie sind die Bestimmung der Weich- und Hartgewebe im Bereich des oberen Luftweges und der Mundhöhle in horizontaler Körperposition, die Darstellung von geschlechtsspezifischen Unterschieden im Bereich des oberen Luftweges sowie die Beschreibung der Anordnung des aufsteigenden Astes des Unterkiefers in der horizontaler Ebene unter Zuhilfenahme des DVT. 


\subsection{Anatomie des orofazialen Systems}

Das orofaziale System besteht aus den Pharyngen (Epipharynx bzw. Nasopharynx, Mesopharynx bzw. Oropharynx und Hypopharynx) sowie der Mundhöhle mit ihren Begrenzungen (Lippen, Zähnen, Gaumen, Kiefer und Zunge), der Nasenhöhle und dem Kehlkopf.

\subsubsection{Systematische Anatomie}

\subsubsection{Pharyngeale Strukturen}

Als Pharynx (Schlund oder Rachen) wird der Raum bezeichnet, der hinter dem Mund und der Nase liegt. Er stellt einen gemeinsamen Abschnitt des Speise- und Luftweges dar. Der Pharynx wird kranial von der Schädelbasis (in diesem Bereich gebildet vom Os sphenoidale, Os occipitale und von der Felsenbeinpyramide) und dorsal von der Halswirbelsäule begrenzt. Er ist circa $12-14 \mathrm{~cm}$ lang und reicht bis zum Ösophagus. Vom Kehlkopf ist er durch den Aditus laryngis, von der Nasenhöhle durch die Choanen und von der Mundhöhle durch den Isthmus getrennt. Im Bereich des Pharynx kommen quergestreifte Muskelgruppen, die in Schlundschnürer (Konstriktoren) und in Schlundheber (Levatoren) eingeteilt werden, vor (Moll und Moll 2006). Die Nervenversorgung des Pharynx erfolgt über den Plexus pharyngeus, der von Fasern aus den Nervus (N.) glossopharyngeus und N. vagus gebildet wird, wobei der Nasopharynx überwiegend durch den N. glossopharyngeus, der Hypopharynx überwiegend durch den N. vagus versorgt wird (Czerny und Formanek 2000). Die Gefäßversorgung des Pharynx erfolgt über die Arteria (A.) pharyngea ascendens, Äste der Arteriae (Aa.) thyroidea superior und inferior und der A. lingualis (Moll und Moll 2006).

Der Pharynx (Abb. 1) wird unterteilt in: 1. Epipharynx,

2. Mesopharynx und

3. Hypopharynx. 


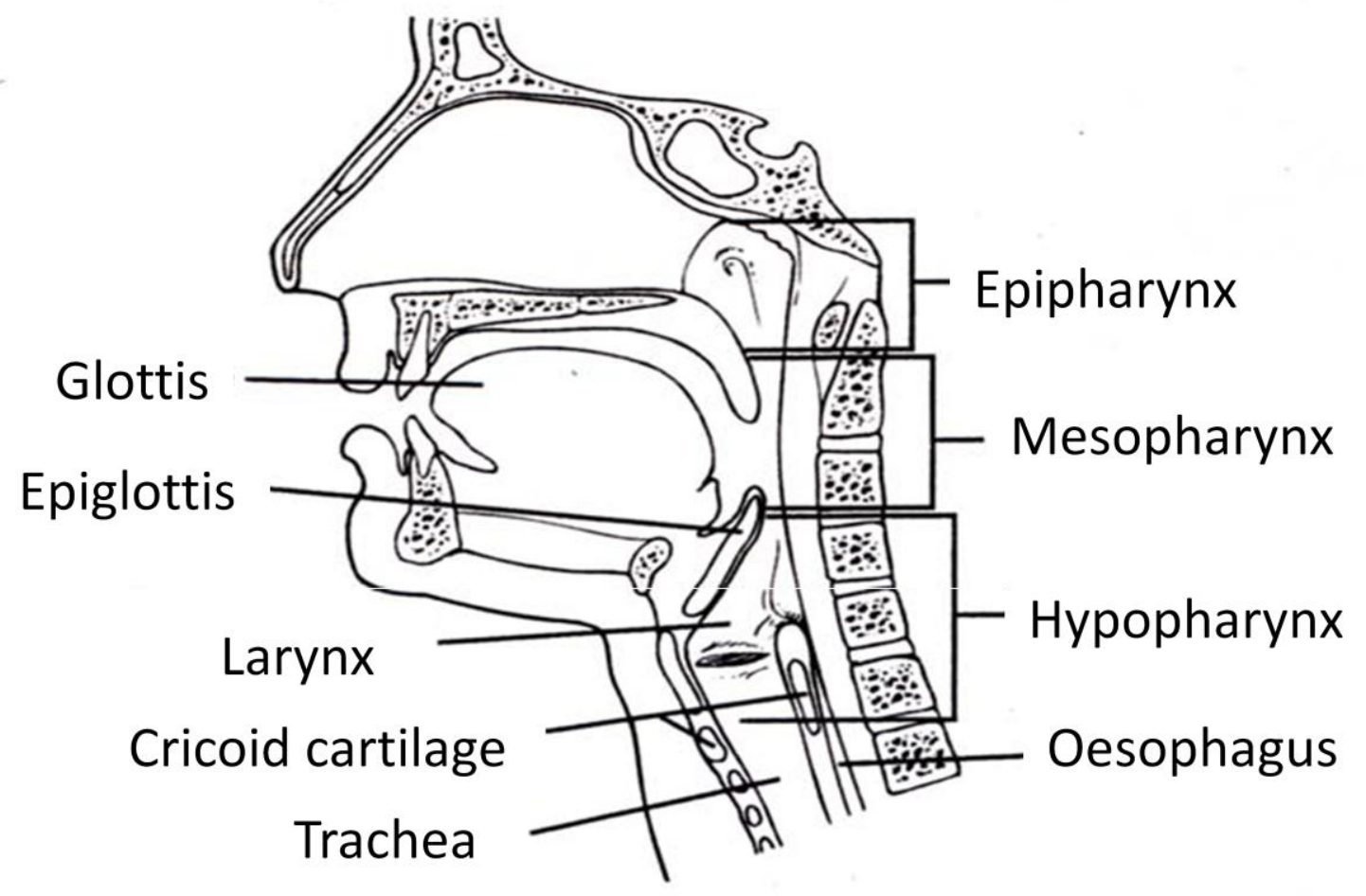

Abb. 1: Sagittales Schema mit Einteilung des Pharynx und angrenzenden Strukturen entsprechend der anatomischen Lokalisation. Modifiziert nach Anderson (2000) S. 1368

Der Epipharynx (Nasopharynx) reicht von der Schädelbasis bis zum freien Rand des Gaumensegels. Sie steht durch die Choanen (hintere Nasenöffnung) mit der Nasenhöhle in Verbindung. An ihrer lateralen Vorderwand liegt die Öffnung der Tuba auditiva (Ohrtrompete), das sogenannte Ostium pharyngeum tubae auditivae, das teilweise von einem Tubenwulst (Torus tubarius) und einem darunter liegenden Tubenknorpel umgeben ist. Die Tuba auditiva verbindet den Pharynx mit dem Mittelohr. Vom Tubenknorpel zieht eine auf dem Musculus (M.) salpingopharyngeus liegende Falte, die Plica salpingopharyngea, schräg abwärts zur seitlichen Pharynxwand. Von unten zieht der Torus levatorius, ein vom M. levator veli palatini aufgeworfener Wulst, zur Tubenöffnung. Am Rand der Tubenöffnung liegt unter der Schleimhaut die Tonsilla tubaria (Tubenmandel). Die Tonsilla tubaria besitzt keine eigene Organmandel (Moll und Moll 2006). Da der Epipharynx ein reiner Luftweg ist, ist er mit respiratorischem Epithel ausgekleidet. Die Schleimhaut besitzt seromuköse Drüsen und enthält am Rachendach als lymphatisches Gewebe die Tonsilla pharyngea (Lüllmann-Rauch 2006). Der Mesopharynx (Oropharynx) erstreckt sich von der Uvula (Gaumenzäpfchen) bis zur Epiglottis (Kehldeckel) (Moll und Moll 2006). Dieser Abschnitt wird gemeinsam von Speise und Atemluft benutzt. Eine deutliche Grenze gegenüber den beiden benachbarten Pharynxanteilen besteht nicht. In den Mesopharynx mündet die Mundhöhle. Den Eingang umgeben der Isthmus faucium mit der Tonsilla palatina (Gaumenmandeln) und der Zungengrund (Schiebler und Korf 2007). Die Hinterwand wird vom 2. und 3. Halswirbel mit der Fascia praevertebralis gebildet. Eine seitliche 


\section{Einleitung}

Begrenzung besteht durch die Gaumenbögen mit den dazwischenliegenden Gaumenmandeln (Probst et al. 2004). Die Zungenwurzel übernimmt Aufgaben der fehlenden vorderen Rachenwand. Von ihr ziehen drei Falten zum Kehldeckel, die Plica glossoepiglottica und die beiden Plicae glossoepiglotticae laterales. Zwischen den drei Falten sinken zwei Gruben ein, die Valleculae epiglotticae (Lippert 2006). Der Mesopharynx gehört gleichzeitig zum Luft- und Speiseweg und ist mit unverhorntem Plattenepithel ausgekleidet (Lüllmann-Rauch 2006).

Der Hypopharynx (Pars laryngea pharyngis) liegt, wie seine anatomische Bezeichnung impliziert, in unmittelbarer Nähe zum Larynx. Topographisch beginnt er medial am Rand der Epiglottis und lateral auf Höhe der Plicae aryepiglotticae. Er enthält sowohl den Zugang zum Kehlkopf als auch zur Speiseröhre. Der Zugang zum Kehlkopf (Aditus laryngis) kann durch Kippung der Epiglottis bei gleichzeitiger Larynxhebung verschlossen werden. Die anatomische Begrenzung zum Ösophagus bildet der pharyngoösophageale Sphinkter, der hinter dem unteren Rand des Ringknorpels (Cartilago cricoidea) in Höhe des 5.-6. Halswirbels gelegen ist (Ekberg 1989, Curtis 1986). Wie der Mesopharynx ist der Hypopharynx gleichzeitig Luft- und Speiseweg und ist ebenfalls mit unverhorntem Plattenepithel ausgekleidet (Lüllmann-Rauch 2006).

Die Schlundschnürer bilden einen nicht ganz geschlossen Muskelring um den Schlund. Zu ihnen zählen M. constrictor pharyngis superior (oberer Schlundschnürer), M. constrictor pharyngis medius (mittlerer Schlundschnürer) und M. constrictor pharyngis inferior (unterer Schlundschnürer). Alle drei Schlundschnürer setzen dorsal an der in der Medianebene des Pharynx liegenden Raphe pharyngis an. Die Raphe ist ein Sehnenstreifen, der am Os sphenoidale dicht vor dem Foramen magnum ansetzt, wodurch der Pharynx an der Schädelbasis aufgehängt ist. Die Schlundschnürer engen den Rachenraum ein und heben den Kehlkopf und das Zungenbein an. Sie werden motorisch von den beiden Plexus pharyngei innerviert, der von Fasern aus dem N. glossopharyngeus, dem N. vagus und sympathischen Nervenfasern gebildet wird.

$\mathrm{Zu}$ den Schlundhebern zählen der M. stylopharyngeus, M. salpingopharyngeus und der M. palatopharyngeus. Sie heben und erweitern den Schlund und werden vom N. glossopharyngeus innerviert (Moll und Moll 2006).

\subsubsection{Orale Strukturen}

Der Gaumen (Palatum) trennt die beiden Nasenhöhlen von der Mundhöhle und bildet das Dach der Mundhöhle. Er wird unterteilt in den harten Gaumen (Palatum durum) und in den weichen Gaumen (Palatum molle). Der harte Gaumen umfasst die vorderen 2/3 des Gaumens. Er besteht aus dem 


\section{Einleitung}

Processus palatinus der Maxilla und der Lamina horizontalis des Os palatinums (Gaumenbein). Im vorderen Gaumenbereich liegen einige quer verlaufende Schleimhautkämme (Plicae palatinae transversae), gegen die die Zunge die weiche Nahrung presst und sie so zerreibt (Moll und Moll 2006).

Der weiche Gaumen füllt das hintere 1/3 des Gaumens aus. Die Grundlage des weichen Gaumens bildet die Gaumenaponeurose (Aponeurosis palatina), in die die Gaumenmuskeln einstrahlen. Der weiche Gaumen bildet die beiden Gaumensegel (Vela palatina), die am Gaumenzäpfchen (Uvula) miteinander in Verbindung stehen (Moll und Moll 2006). Das Gaumensegel ist eine mit Schleimhaut bekleidete Muskelplatte, die proximal am Hinterrand des harten Gaumens ihren Ursprung findet. Seitlich wirft das Velum palatinum zwei Schleimhautfalten auf, den vorderen Arcus palatoglossus und den hinteren Arcus palatopharyngeus. Diese beiden Gaumenbögen werden von den gleichnamigen Muskeln gebildet, weitere Muskeln sind M. levator veli palatini, M. tensor veli palatini und M. uvulae. Auf ihrer Höhe befindet sich der Isthmus faucium, d.h. Übergang zwischen Mundhöhle und Rachen (Mesopharynx) (Oettinger und Oettinger 1992). Zwischen den beiden Gaumenbögen liegt in einer Nische (Tonsillarbucht) die Tonsilla palatina. Sie gehört neben der Tonsilla lingualis, der Tonsilla pharyngealis und der Tonsilla tubaria mit zum lymphatischen Rachenring (Waldeyer-Rachenring), der im Bereich des Übergangs von der Mund- und Nasenhöhle in den Pharynx angesiedelt ist (Moll und Moll 2006).

Der harte Gaumen wird sensibel vom N. palatinus major, der weiche Gaumen von den Nn. palatini minores (alle Äste des N. maxillaris) innerviert. Die Gefäßversorgung erfolgt über die A. palatina, A. pharyngea ascendens und A. lingualis (Moll und Moll 2006).

Die Mandibula besteht aus dem Corpus mandibulae und einem beidseits im dorsalen Bereich gelegenen aufsteigenden Ast, dem Ramus mandibulae. An diesem befindet sich jeweils ein Processus coronoideus, welcher dem Musculus temporalis als Ansatz dient. Ein weiterer Bestandteil des aufsteigenden Astes ist der Gelenkfortsatz (Processus condylaris) mit dem Kiefergelenksköpfchen (Caput mandibulae), der das Kiefergelenk bildet (Drenckhahn und Zenker 2002). An der Innenseite des Unterkieferastes sitzt eine Knochenzunge, die das Foramen mandibulae überdeckt. Diese Öffnung ist die Eintrittsstelle des Nervus alveolaris inferior, einer der drei Hauptäste des Nervus trigeminus. Der Nerv verläuft im Unterkieferkanal (Canalis mandibulae) in doppel-S-förmiger Krümmung, mehr auf der lingualen Seite des Unterkiefers gelegen, unter den Wurzelspitzen und innerviert die Zahnfächer und die entsprechenden Zähne sensibel (Reich 1980). In der Sagittalebene verläuft der Kanal in mäßigem Gefälle von distal kranial nach mesial kaudal. Im Bereich des 1. Prämolaren wendet er sich dann in sanftem Bogen nach kranial, sodass die Form eines Krückstockgriffes entsteht und endet am Foramen mentale (Schug-Kösters 1968). Der Endast verlässt als Nervus mentalis den Unterkieferkörper am gleichnamigen Foramen mentale. Die 


\section{Einleitung}

Blutversorgung des Unterkiefers erfolgt über die A. alveolaris inferior (Reich 1980, Drenckhahn und Zenker 2002, Moll und Moll 2006).

Das optimale Gebiss, hinsichtlich auf Morphologie und Funktion, wird unter dem Begriff Eugnathie zusammengefasst. Eine Eugnathie besteht, wenn der SNB Winkel zwischen $78-80^{\circ}$ liegt. Ist der Winkel größer als $80^{\circ}$ besteht eine Prognathie, bei einem Winkel unter $78^{\circ}$ liegt eine Retrognathie vor. Desweitern beinhaltet sie eine Harmonie der Muskulatur und der Kiefergelenke, eine ungehinderte Okklusion und Artikulation, eine vollständige Zahnzahl bei normaler Zahnform und gut ausgeformte Zahnbögen, normale horizontale und vertikale Position der Zähne bei einer neutralen Okklusion (Angle-Klasse I) (Schopf 2008). Bei der Angel Klasse I sind die vorderen Höcker des oberen Sechsjahrmolaren zwischen den großen Höckern des unteren Sechsjahrmolaren okkludiert. Abweichungen davon werden als Angle Klasse II oder III bezeichnet.

Man kann bei der vertikalen Kieferrelation eine physiologischer und physikalischer Bisshöhe unterscheiden. Unter der physiologische Bisshöhe versteht man die unbewusste Abstandhaltung des Unterkiefers zum Oberkiefer bei geschlossenen Lippen. Durch die Schwerkraft ist sie vom Aktivitätszustand der Adduktoren abhängig. Bei retrudierter Kontaktpunktposition befindet sich der Unterkiefers relativ zum Oberkiefer in der physikalischer Bisshöhe (Hupfauf 1971, Koeck und Bierwirth 1986). Die natürlichen Zähne geben die Okklusion die vertikale Lage des Unterkiefers vor. Der Interokklusalabstand ermöglicht der Muskulatur einen Ruhetonus, ohne das die Ruheschwebelage durch die okklusale Kontakte gestört wird (Lotzmann 1998).

Bei der horizontalen Unterkieferrelation befinden sich die Unterkieferkondylen an der kranioventralsten Position gegenüber dem hinteren Abhang des Tuberculum articulare in der Fossa mandibularis (Ash und Nelsn 2003). Einen Einfluss auf die zentrische Kondylenlage haben u.a. die Stellung des Kopfes zum Rumpf, die psychisch- emotionale Verfassung, den Muskeltonus (Disstress; Müdigkeit), die Tageszeit (Shafagh et al. 1975; Latta 1992), propriozeptive Einflüsse (Desmodont, Kaumuskulatur) und vorhandene Schmerzen (Obrez und Stohler 1996; Obrez und Türp 1998).

Das am Übergang vom Mundboden zum Hals tastbare hufeisenförmige Zungenbein (Os hyoideum), liegt bei normaler Kopfhaltung in der Höhe des 4. Halswirbels. Das Os hyoideum besteht aus einem Körper (Corpus) und auf jeder Seite einem großen Horn (Cornu majus) und einem kleinen Horn (Cornu minus). Das Zungenbein ist ein freier, muskulär zwischen dem Schädel sowie dem Sternum und dem Schildknorpel aufgehängter Knochen, der keine Gelenkverbindung zu anderen Knochen besitzt. 


\section{Einleitung}

Die kraniale Begrenzung der Mundhöhle bilden die knöchernen und weichen Strukturen des Gaumens. Nach lateral und ventral ist sie durch die Zähne mit dem Alveolarkamm und Wangen bzw. Lippen begrenzt.

Die lateral der Zahnreihen in den Wangen liegende Kaumuskulatur hängt den Mandibularkörper beweglich auf und hält ihn in Position. Sie besteht aus dem M. masseter, dem M. temporalis und den Musculi (Mm.) pterygoidei. Sie sind für die dreidimensionale Beweglichkeit des Unterkiefers verantwortlich und die Hauptmuskulatur der Kaubewegung. In Einheit mit der mimischen Muskulatur positionieren sie beim Kauakt den Bolus und sorgen auch für korrekte Artikulation.

Zwischen Mandibula und dem Hyoid bilden die sich als passive Strukturen trapezförmig aufspannenden Mundbodenmuskeln die kaudale Grenze. $\mathrm{Zu}$ ihnen zählen M. mylohyoideus, M. geniohyoideus und der Venter anterior des M. digastricus (Moll und Moll 2006).

Die Zunge ist ein festes mit der Schleimhaut bedecktes Organ. Die sie bildenden Muskeln entspringen zum größten Teil der bindegewebigen Aponeurosis linguae. Sie wird in innere und äußere Muskulatur unterschieden. Zur inneren Muskulatur gehören der M. longitudinalis superior, der M. inferior linguae, der M. transversus linguae und der M. verticalis linguae. Zu den äußeren Zungenmuskeln zählen der M. genioglossus, der M. hyoglossus und der M. styloglossus.

Durch die dreidimensionale Anordnung der Muskulatur ist die Zunge außerordentlich beweglich. Sie kann sich bei Aktivität der Binnenmuskulatur verkürzen, verlängern, verschmälern oder runden. Die äußeren oberen Skelettmuskeln sorgen bei Aktivierung vor allem für die Lageveränderung der Zunge im Mundraum (Moll und Moll 2006).

Die Flexibilität der Zunge ermöglicht eine Vielzahl an Funktionen: Sprache und Lautbildung, Boluspositionierung bei der Nahrungszerkleinerung und Schlucken.

\subsubsection{Funktionelle Anatomie nach dem Funktionskompartimentmodell}

Das oronasopharyngeale System besteht aus Mund, Nase und Rachen sowie angrenzenden Strukturen des Viszerokraniums. Die funktionellen Einheiten bilden die Wände und den Inhalt des Hohlraumsystems. Die Abbildung 2 zeigt eine schematischen Darstellung der Funktionseinheiten des oronasopharyngealen Systems nach Engelke. 


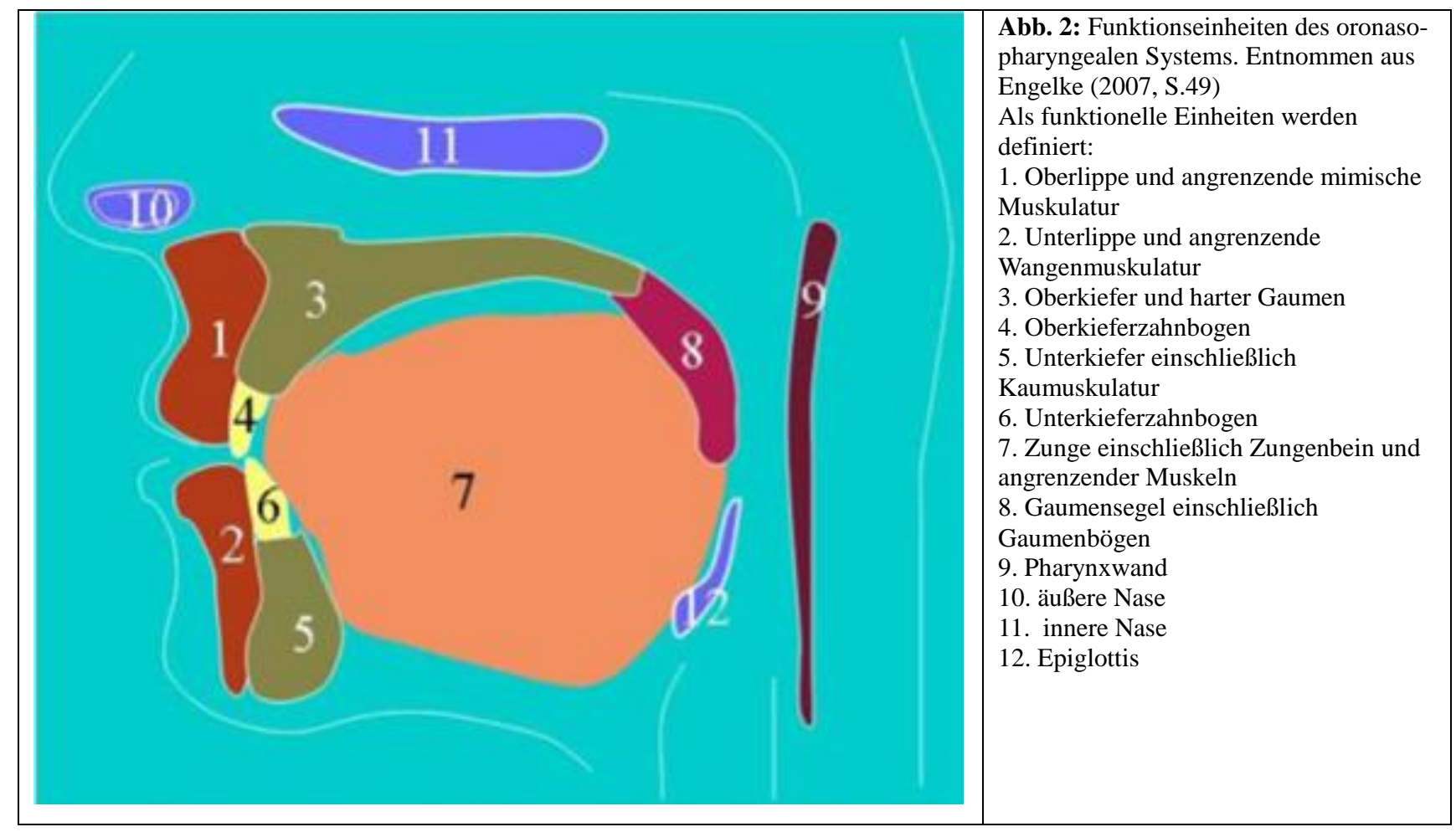

Des Weiteren können 4 Funktionskompartimente definiert werden, die unter der Funktion abzugrenzen sind.

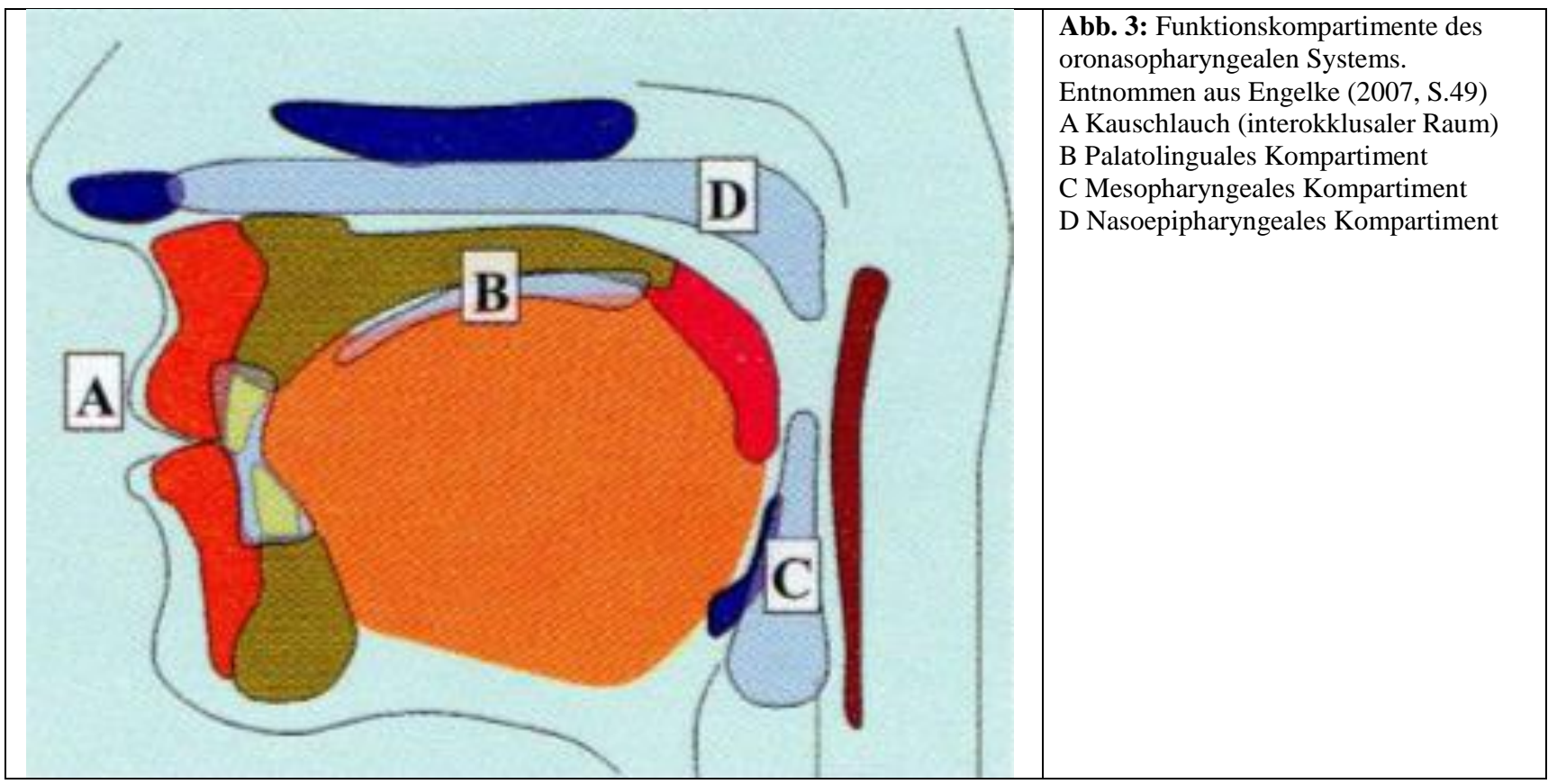

Das Funktionskompartiment A entspricht dem Kauschlauch. Der Kauschlauch wird definiert als der

Raum, der die Zahnreihe mit dem angrenzenden Alveolarfortsatz beider Zahnreihen umgibt (Abb. $3)$.

Eine sinnvolle Definition des Kauschlauches ist nur möglich, wenn die Zunge randständig an der Basis des Oberkieferalveolarfortsatzes liegt. Bei geöffnetem Mund kann der Kauschlauch nicht von der freien Mundhöhle abgegrenzt werden. 


\section{Einleitung}

Das Funktionskompartiment B wird vom Gaumendach, Gaumensegel, dem Zungenrücken und dem Oberkieferalveolarfortsatz umschlossen. Es ist nur vom Funktionskompartiment A abzugrenzen, wenn sich der Zungenrücken oberhalb der Okklusionsebene befindet und der Zungenrand rings dem Oberkieferalveolarfortsatz anliegt. Wird der Mund geöffnet, verschmelzen die Kompartimente A und B miteinander. Durch das vollständige Anliegen des Velums an dem Zungenrücken werden Kompartimente B und C getrennt (Abb. 3).

Das Funktionskompartiment $\mathrm{C}$ entspricht dem mesopharyngealen Raum. Bei Abheben des Velums vom Zungenrücken bilden die Kompartimente B und C eine Einheit. Nach unten wird das Kompartiment durch den Kehlkopf begrenzt, dabei stabilisiert die Epiglottis den unteren Abschnitt des Kompartimentes. Oberhalb der Epiglottis ist der mesopharyngeale Raum kollapsfähig, dort spielen sich Obstruktionen und Schnarchlautbildung ab.

Zur Nase hin schließt sich das Funktionskompartiment D an. Das Lumen der äußeren Nase, der Nasennebenhöhlen und des epipharyngealen Raumes bilden das Funktionskompartiment D. Aufgrund seiner Struktur weist dieses Kompartiment keine Kollapstendenz auf (Engelke 2007) (Abb. 3).

\subsubsection{Röntgenologische Anatomie mittels Fernröntgenseitenbild (Kephalometrie)}

Mittels Fernröntgenseitenbilder können neben dem Hartgewebe (Knochen und Zähne) auch Weichgewebe dargestellt werden. Diverse Studien zeigen, dass mit Hilfe des Fernröntgenseitenbild Weichgewebspunkte im Bereich des Epi-, Meso- und Hypopharynx ermittelt werden können und so die Anatomie des Pharynx beschrieben werden kann. Als Weichgewebe können die Pharynxhinterwand, der Zungenrücken, die Epiglottisspitze und der weiche Gaumen mit der Uvulaspitze identifiziert werden. Dementsprechend kann die Weichgaumenlänge und -breite, die Strecke von der Epiglottisspitze zur Spina nasalis posterior (= kollapsfähige Segment), der Luftweg auf Höhe des weichen Gaumens, auf Höhe des dorsalsten Punktes der Zunge, auf Höhe der Okklusionsebene, auf Höhe der Spina nasalis posterior sowie auf Höhe der Vallecula vermessen (Hochban et al. 1994, Tangugsorn et al. 1995, Battagel et al. 2000, Pradel et al. 2000, Johal und Conaghan 2004, Battagel et al. 2007). Außerdem wurden die Flächen vom Oropharynx, der Zunge und des weichen Gaumens auf den Fernröntgenseitenaufnahmen bestimmt (Battagel et al. 2000, Johal und Conaghan 2004, Johal et al. 2007). Die oben angegebenen Studien zeigen, dass der Mesopharynx am häufigsten hinter dem Velum seine engste Stelle (Isthmus) aufweist. Im Bereich des Zungenrückens vergrößert er seinen sagittalen Durchmesser. Allerdings gibt das Fernröntgenseitenbild keine Aussage über dem transversalen Durchmesser. Die Abbildung 4 zeigt eine schematische Darstellung der von verschiedenen Autoren gemessenen Parameter. 


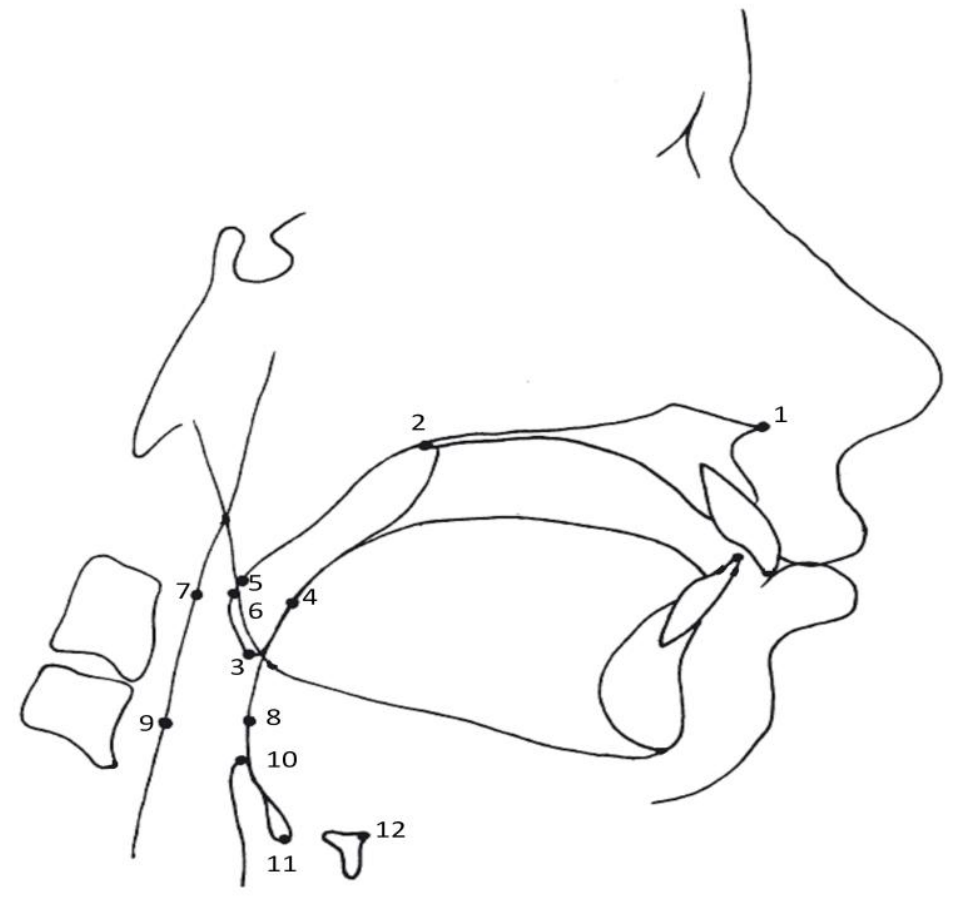

Abb.4: Schematische Darstellung von Hart- und Weichgewebspunkten (modifiziert in Anlehnung an Battagel et al. 2007). 1 Spina nasalis anterior, 2 Spina nasalis posterior, 3 Uvulaspitze, 4 Punkt auf der oralen Seite des Gaumen, wo der Gaumen am breitesten ist, 5 Punkt auf der nasalen Seite des Gaumen, wo der Gaumen am breitesten ist, 6 Punkt auf dem weichen Gaumen, wo der Luftweg hinter dem weichen Gaumen am engsten ist, 7 Punkt an der posterioren Pharynxwand, wo der Luftweg am engsten ist, 8 dorsalste Punkt auf dem Zungenrücken, 9 Punkt an der posterioren Pharynxwand, wo der Luftweg hinter der Zunge am engsten ist, 10 Epiglottisspitze, 11 Vallecula glossoepiglottica, 12 Hyoid

Weiterhin wurden Winkel wie Sella-Nasion-A Punkt (SNA) und Sella-Nasion-B Punkt (SNB) gemessen. Auch wurden die Strecke vom Hyoid zur Mandibularlinie und die Strecke zwischen Spina nasalis anterior (ANS) und Spina nasalis posterior (PNS) bestimmt.

Die Tabelle 1 zeigt einen Überblick von den bisher gemessenen Parametern. 
Tabelle. 1: Überblick über kephalometrische Messwerte.

\begin{tabular}{|l|c|c|c|c|c|c|}
\hline Parameter\Autor & Gungor et al. 2013 & $\begin{array}{l}\text { Johal und } \\
\text { Conaghan 2004 }\end{array}$ & $\begin{array}{l}\text { Battagel et } \\
\text { al. 2000 }\end{array}$ & $\begin{array}{l}\text { Pradel et al. } \\
2000\end{array}$ & $\begin{array}{l}\text { Tangugsorn } \\
\text { et al. 1995 }\end{array}$ & $\begin{array}{l}\text { Hochban et } \\
\text { al. 1994 }\end{array}$ \\
\hline SNA & $\mathrm{X}$ & $\mathrm{X}$ & $\mathrm{X}$ & $\mathrm{X}$ & $\mathrm{X}$ \\
\hline SNB & $\mathrm{X}$ & $\mathrm{X}$ & $\mathrm{X}$ & $\mathrm{X}$ & $\mathrm{X}$ \\
\hline Weichgaumenbreite & $\mathrm{X}$ & $\mathrm{X}$ & $\mathrm{X}$ & & $\mathrm{X}$ & $\mathrm{X}$ \\
\hline Weichgaumenlänge & $\mathrm{X}$ & $\mathrm{X}$ & $\mathrm{X}$ & & $\mathrm{X}$ \\
\hline Hyoid zu Mandibularlinie & $\mathrm{X}$ & & $\mathrm{X}$ & & $\mathrm{X}$ \\
\hline Länge harter Gaumen & & $\mathrm{X}$ & & & $\mathrm{X}$ & \\
\hline $\begin{array}{l}\text { Luftweg hinter dem weichem } \\
\text { Gaumen }\end{array}$ & $\mathrm{X}$ & $\mathrm{X}$ & $\mathrm{X}$ & $\mathrm{X}$ & $\mathrm{X}$ & \\
\hline Luftweg dorsal der Zunge & & & $\mathrm{X}$ & & $\mathrm{X}$ & $\mathrm{X}$ \\
\hline $\begin{array}{l}\text { Luftweg auf Höhe der } \\
\text { Okklusionsebene }\end{array}$ & & & & & $\mathrm{X}$ & $\mathrm{X}$ \\
\hline Luftweg auf Höhe der Vallecula & & & & & $\mathrm{X}$ & $\mathrm{X}$ \\
\hline $\begin{array}{l}\text { Luftweg hinter Spina nasalis } \\
\text { posterior }\end{array}$ & & & & & & $\mathrm{X}$ \\
\hline
\end{tabular}

\subsection{Bildgebende Verfahren zur Darstellung des oberen Luftweges}

\subsubsection{Zweidimensionale Aufnahmen mittels lateralem Fernröntgenseitenbild}

Das Fernröntgenseitenbild ist eine vor allem in der Kieferorthopädie und Mund-, Kiefer- und Gesichtschirurgie verwendete Röntgenaufnahmetechnik. Für die Behandlungsplanung erfolgt die Bestimmung von Wachstumstendenzen, Kiefer- und Knochenrelationen sowie der Achsen der Frontzähne Pasler 2008). Das Fernröntgenseitenbild zur Kephalometrischen Analyse ist eine weit verbreitete Untersuchung bei Patienten mit schlafbezogene Atemstörungen, insbesondere zur Beurteilung der kraniofazialen Morphologie. Aufnahme- und Analysetechnik wurden aus der Mund-, Kiefer- und Gesichtschirurgie und der Kieferorthopädie übernommen (Maurer und Stuck 2008).

Es handelt sich um eine laterolaterale Aufnahme des Schädels mit möglichst parallelem Strahlengang. Der Abstand zwischen Strahlenquelle und Röntgenfilm beträgt deshalb etwa 1,5 Meter, der Abstand zwischen Kopf und Röntgenfilm wird möglichst gering gewählt. Die Aufnahmedauer beträgt ca. 12 Sekunden. Während dieser Zeit fahren zwei Blenden mit einem vertikalen Schlitz synchron über die Strahlenquelle und den Film. Dies führt dazu, dass der Film in einer vertikalen Linie von ventral nach dorsal belichtet wird. Durch die Benutzung von sog. Weichteilfilter können die einzelnen Abschnitte des Röntgenbildes unterschiedlich stark belichtet werden. Daher sind auf ein und demselben Bild sowohl die relativ strahlendurchlässigen Weichgewebe des Gesichtsprofils, als auch die relativ röntgenopaken Knochen zu sehen (Pasler 2008). 


\section{Einleitung}

Die Aufnahme erfolgt entweder mittels konventionellem Röntgenfilm oder mit einem digitalen Sensor. Die Strahlenbelastung je Aufnahme hängt von der Größe des Patienten (des zu durchstrahlenden Gewebes) und der verwendeten Technik ab. Durch Verwendung digitaler Röntgentechnik kann die Strahlenbelastung beim FRS halbiert werden. Die effektive Dosis wird 2,3 $\mu \mathrm{Sv}$ für konventionelle und 1,1 $\mu \mathrm{Sv}$ für digitale Aufnahmen angegeben (Visser et al. 2001). Durch die Entwicklung immer empfindlicherer Sensoren werden sowohl die Aufnahmen qualitativ, als auch die benötigte Strahlendosis geringer. Der Kopf des Patienten ist in einem Cephalostat fixiert. Eine Einschränkung des seitlichen Fernröntgens ist, dass man einen dreidimensionalen Raum zweidimensional darstellt. Man erhält ein unvollständiges Bild, da die transversale Dimension des Luftweges nicht abgebildet wird (Hochban et al. 1994, Solow et al. 1996, Battagel et al. 2000). Des Weiteren werden durch den Summationseffekt der Röntgenstrahlen die Gewebestrukturen des gefragten Raumes zweidimensional und deshalb übereinander projiziert aufgezeichnet (Pasler 2008). Ein weiterer negativer Aspekt ist die projektionsbedingte Vergrößerung, die Hochban et al. (1994) mit 10\% angab bei einem Fokus-Filmabstand von $3 \mathrm{~m}$ bei minimalen Objekt-Filmabstand. Beim 1,5 m Fokus-Film-Abstand betragt der Vergrößerungsfaktor 7 \% (Drescher 2000) und beim 4 m Abstand etwa 5,26 \% (Segner und Hasund 1998).

Andere Untersucher betonten jedoch die Fehlermöglichkeiten bei der Auswertung der Fernröntgenseitenaufnahme und stellten deren klinische Bedeutung in Frage (Moyer und Bookstein 1979, Moss 1983). Es konnte deutlich gemacht werden, dass die Identifizierung der kephalometrischen Referenzpunkte als eine der größten Fehlerquellen anzusehen ist (Freisfeld 1973, Houston 1983, Schopf 1994).

\subsubsection{Computertomographie (CT)}

Die Computertomographie ist ein mit Röntgenstrahlen bildgebendes Verfahren zur überlagerungsfreien Darstellung von Transversalschnittbildern mit hoher Kontrastauflösung. Der Engländer G. N. Hounsfield und der Amerikaner A. M. Cormack entwickelten diese Modalität zu Beginn der 1970er Jahre unabhängig voneinander und erhielten für diese Leistung 1979 den Nobelpreis für Medizin. Die Computertomographie ist eine Weiterentwicklung der ursprünglichen Tomographieverfahren, sie stellt das erste vollständig digitale Schnittbildverfahren in der Medizin dar.

Bei diesem Verfahren werden die Daten mit Hilfe spezieller Sensoren aus verschiedenen Richtungen der darzustellenden Körperregionen gesammelt, sodass die räumlichen Beziehungen der absorbierenden Strukturen bestimmt und nach Berechnung dargestellt werden können. 


\section{Einleitung}

Die Detektoren zur Erfassung der Absorptionsdifferenzen sind wie der Film bzw. die Folie bei der Orthopantomographie gegenüber der Röntgenröhre hinter dem Patienten angeordnet. Für die Bildqualität und Auflösung ist die Anzahl der Schwächungsmessungen aus unterschiedlichen Richtungen ausschlaggebend, d.h. je mehr Messungen aus unterschiedlichen Richtungen, desto größer sind Anzahl und Informationsgehalt der einzelnen Bildelemente. Es können also röntgenologische Bildinformationen über einen Querschnitt durch den Patienten erhoben werden, ohne dass Bildüberlagerungen entstehen (Pickuth et al. 1998, Novelline 2001).

Als Standardmethode der Schnittbildverfahren galt über ein Jahrzehnt die Computertomographie. Durch die Einführung der Spiral-CT-Technik ergaben sich Verbesserungen im Hinblick auf die Untersuchungsdauer, die Reduktion von bewegungsbedingten Artefakten und die Möglichkeit multiplanarer Bildrekonstruktionen (Lomoschitz et al. 2000). Bei der Spiraltomographie rotiert die Röntgenröhre um den Patienten, während dieser mit kontinuierlichem Vorschub entlang seiner Längsachse durch den fächerförmigen Strahlengang bewegt wird (Pasler 2008) (Abb.5).

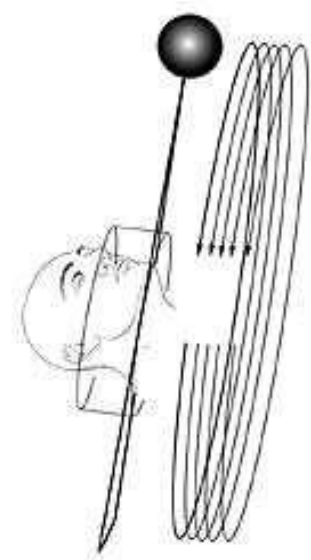

Abb. 5: Funktionsprinzip der Spiral-Computertomographie. Entnommen aus Hirsch (2002)

Die entstehenden Schichten bestehen aus einzelnen rasterförmig angeordneten Volumenpunkten (Voxel), deren Höhe durch die jeweilige Schichtdicke vorgegeben ist. Jedem Voxel wird während des Scans ein entsprechender Absorptionskoeffizient zugerechnet, der in einem Zahlenwert ausgedrückt werden kann.

Der jeweilige Absorptionswert wird in Beziehung zum Absorptionskoeffizienten $\mu$ von Wasser gesetzt und nach einem der beiden Erstbeschreiber als sogenannte Hounsfield-Einheit (HE) angegeben. Diese Werte sind charakteristisch für die jeweiligen Gewebe des menschlichen Körpers. Die CT in der Mund-, Kiefer- und Gesichtschirurgie bzw. in der Zahnmedizin ist aus strahlenhygienischen Gründen nur mit der richtigen Indikationsstellung, d.h. bei komplexen Fragestellungen des Gesichtsschädels und in der Tumordiagnostik anzuwenden (Fuhrmann et al. 2003). CT-Untersuchungen führen zu ca. $35 \%$ der medizinisch bedingten Strahlenbelastung der 


\section{Einleitung}

Bevölkerung, obwohl ihr Anteil an Röntgenuntersuchungen nur 3\% beträgt (Kaul et al. 1997, Galanski et al. 2001, Brugmans et al. 2002, Prokop 2002).

Schnelle (2001) stellte bei Dosismessungen an einem Phantomkopf bei der CT-Untersuchung mit einer Röhrenspannung von $140 \mathrm{kV}$ und einer Röhrenstromstärke von 94 mA Maximalwerte von 23 mSv, beim sogenannten dosisreduzierten CT (60 mA) $11 \mathrm{mSv}$ und $6 \mathrm{mSv}$ (43 mA) fest, jeweils gemessen im Bereich der oberen ersten Molaren.

Cohnen et al. (2002) stellten bei Dosismessungen an einem Phantom Ortsdosiswerte von 4,06 mSv und 2,44 mSv bei einer CT-Untersuchung mit einer Stromstärke von $94 \mathrm{~mA}, 2,54 \mathrm{mSv}$ und 1,22 $\mathrm{mSv}$ mit einer Stromstärke von $60 \mathrm{~mA}$ und 1,03 mSv und 0,7 mSv mit einer Stromstärke von 43 mA jeweils für die Schilddrüse und die Augenlinse fest. Die Werte des Niedrigdosis-CTs liegen damit im Bereich der Strahlendosis der digitalen Volumentomographie. Eine neue Studie von Jeong et al. (2012) gab für die Abbildung des Unterkiefers mit einem Multi-Detektor CT eine effektive Dosis von 199-425 $\mu \mathrm{Sv}$ an.

Hanazawa et al. (2004) maßen den Abstandes des Canalis mandibulae zum krestalen Rand der Mandibula. Er zeigte in einer vergleichenden Untersuchung dreidimensionaler Röntgengeräte beim herkömmlichen CT eine Messgenauigkeit von $\pm 1 \mathrm{~mm}$ in 93,7\% der Messungen. Hübinger (2008) konnte in seiner Studie ebenfalls diese Ergebnisse bestätigen.

Im Vergleich zum lateralen Fernröntgenseitenbild ist der Gewebekontrast mit der CT signifikant besser. Außerdem ermöglicht das CT präzise Messungen von Querschnitten in verschiedenen Ebenen des Atemwegs, dreidimensionale Rekonstruktionen und Volumenbestimmungen (Maurer und Stuck 2008).

Fairburn et al. (2007) stellten den oberen Luftweg mittels Spiral-CT dar. Sie vermaßen den Pharynx in der mediosagittalen und in der transversalen Richtung alle $10 \mathrm{~mm}$ ausgehend von der harten Gaumenebene, um Veränderungen der Morphologie des Luftweges nach chirurgischer Vorverlagerung des Oberkiefers und Unterkiefers zu analysieren.

Fajdiga (2005) maß die pharyngealen Strecken (anterior-posterior und transversal) und Flächen auf Höhe vom harten Gaumen, der engsten Stelle hinter dem weichen Gaumen und auf Höhe des Zungengrundes. Weiterhin wurden die Dicke und die Länge des weichen Gaumens vermessen. Ziel der Studie war, klinisch erkennbare Gründe für das Schnarchen zu ermitteln.

\subsubsection{Digitale Volumentomographie (DVT)}

Diese 1997 entwickelte Aufnahmetechnik in der Zahn-, Mund- und Kieferheilkunde bietet die gleichen Rekonstruktionsmöglichkeiten für die Hartgewebsdiagnostik wie die Spiraltechnik in der Computertomographie, scheint aber mit einer geringeren Strahlenexposition verbunden zu sein 
(Mozzo et al. 1998, Arai et al. 1999, Moebes et al. 2000). Sie wurde zur Darstellung der knöchernen Strukturen am menschlichen Schädel entwickelt, wobei gleichzeitig die hohe Strahlenbelastung der CT vermieden werden sollte (Cohnen et al. 2002, Scarfe et al. 2006).

Bei der Digitalen Volumentomographie wird, im Gegensatz zum CT, das stationäre Objekt durch eine einmalige Rotation $\left(360^{\circ}\right)$ der Sender-Detektoreinheit mit einem kegelförmigen Strahlenbündel (Conebeam-Technik) erfasst (Goch 2005, Voßhans 2005) (Abb. 5).

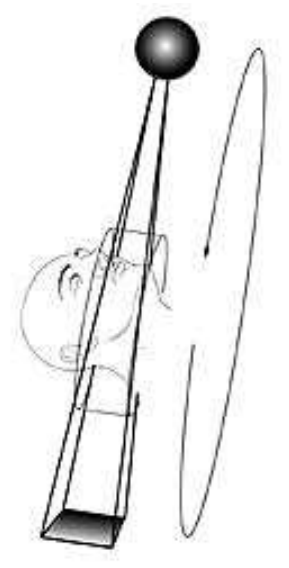

Abb. 6: Funktionsprinzip der Digitalen Volumentomographie (DVT). Entnommen aus Hirsch (2002)

Bei der Aufnahme führt das Gerät bei jedem Grad eine Messung durch. Die tatsächliche Zeit der Strahlenexposition beträgt Aufgrund des gepulsten Röntgenstrahls nur einen Bruchteil der Umlaufzeit, die restliche Zeit dient der Ausrichtung der Röntgenröhre.

Die Expositionszeit ist durch diese Röntgentechnik bei den Geräten wesentlich geringer, als bei Standard-CT-Aufnahmen, wie sie in der Klinik zur Anwendung kommen (Rother 2006).

Anschließend wird aus 360 einzelnen Projektionsradiographien ein dreidimensionaler Datensatz rekonstruiert. Es lassen sich beliebig viele Primärrekonstruktionen aus einem Rohdatensatz erstellen, da dieser archiviert wird.

Die Digitale Volumentomographie eignet sich nicht zur Weichteildiagnostik, insbesondere wegen der Strahlenqualität und der Rauschanteile (Thiel und Hassfeld 2001), erfüllt allerdings alle Anforderungen an die Hartgewebsdiagnostik im Zahn-, Mund- und Kieferbereich (Mozzo et al. 1998, Moebes et al. 2000). Vor allem zu prächirurgischen Abklärungen bei Zysten, Kieferhöhlenveränderungen, Weisheitszahnextraktionen oder zur präimplantorischen Planung wird das DVT eingesetzt. Im Gegensatz zur Computertomographie werden hier keine HounsfieldEinheiten berechnet.

Die Daten der Primärrekonstruktion können zusätzlich im Dicom-Format ausgegeben und so in Programme zur computerunterstützten Implantologie eingelesen werden.

Nach Mozzo et al. (1998) beträgt die Strahlendosis nur etwa ein Sechstel einer herkömmlichen Computertomographie. Strocchi et al. (2012) stellte fest, dass die effektive Dosis bei der Multi- 


\section{Einleitung}

Detektor CT 50\% über der des DVT liegt. Jeong et al. (2012) gaben für die Abbildung des Unterkiefers eine effektive Dosis von 83-332 $\mu$ Sv an. Moebes et al. (2000) konstatierten, dass die effektive Dosis bei der digitalen Volumentomographie im Bereich von Niedrigdosis-CT-Scans mit 43 mA liegt. Suomalainen et al. (2008) stellten hierzu Versuche an, die Dosiswerte des MultisliceCT den Werten des DVT anzugleichen. Das Resultat war eine signifikante Verschlechterung der Darstellungsgenauigkeit von Multislice-CT gegenüber dem DVT. Auch andere Autoren konnten durch Reduktion der Röhrenstromstärke bei CT Geräten das Erreichen vergleichbarer DVTDosiswerte nachweisen, was jedoch auch hier den nachteiligen Effekt der Verschlechterung der Bildqualität mit sich brachte (Schnelle 2001). Wie unschwer zu erkennen ist, ist die Anzahl von Studien, die über Strahlenbelastung veröffentlich wurden sehr groß. Allerdings sind die Studien schwer miteinander zu vergleichen. Sie wurden oft mit unterschiedlichen Geräten und Einstellungen erstellt. Je kleiner das geröntgte Volumen, je geringer das Auflösungsvermögen und je geringer der Kontrast desto niedriger die Strahlenbelastung.

Mit der DVT-Aufnahmetechnik ist eine Reduktion der Strahlenbelastung ohne großen Verlust der Darstellungsgenauigkeit im Vergleich zum konventionellen CT möglich (Rother 2006). So kann eine bis zu 15-fache Reduktion der CT-Werte durch die neue Technik erreicht werden (Scarfe et al. 2006).

Demgegenüber stehen auch hier wieder Aussagen von Autoren, die den Einsatzbereich des DVT, bedingt durch die vermeintlich reduzierte Strahlenexposition, im Vergleich zum CT eher als begrenzt ansehen. So berichten Frank und Frank (2007) von einer auch bei dieser Technik auftretenden reduzierten Bildqualität hinsichtlich der Auflösung, unabhängig von der Verzerrung. Goch stellte 2005 in ihrer Studie zur Messgenauigkeit zwischen den CT-Untersuchungen und den DVT-Untersuchungen keine signifikanten Unterschiede fest. Für die DVT-Untersuchungswerte ergaben sich im Mittel Streckenabweichungen von $-0,22 \mathrm{~mm}$ im Vergleich zum anatomischen Modell.

Die zurzeit auf dem Markt befindlichen Modelle unterscheiden sich in der Patientenpositionierung (liegend oder aufrecht), in der Aufnahmedauer und Größe des Scanbereiches (Scherer et al. 2007). Die Größe des Scanbereiches der derzeitig angebotenen Geräte variiert zwischen 3x4 cm (Morita 3 D Accutiomo) bis zu 20x20 cm (New Tom 3 G-MF 12) (Dirsch 2008).

\subsubsection{Magnetresonanztomographie (MRT)}

Die MRT ist ein computergestütztes bildgebendes Verfahren der Tomographie, das auf dem Prinzip der Magnetresonanz beruht und im Gegensatz zur Computertomographie keine ionisierende Strahlung verwendet (Pschyrembel 2011). Wasserstoffkerne (Protonen), deren Spin in einem 


\section{Einleitung}

Magnetfeld ausgerichtet werden, absorbieren elektromagnetische Wellen definierter Frequenzen. Dabei wird ihre Ausrichtung zum äußeren Magnetfeld gestört, bei der Rückkehr in den ursprünglichen Zustand senden die Wasserstoffkerne ihrerseits messbare Hochfrequenzsignale ab. Von den verschiedenen Geweben im menschlichen Körper werden je nach Wassergehalt sehr typische Signale abgeben, woraus der Computer ein kontrastreiches Bild erstellen kann. Die Software ermöglicht eine räumliche Darstellung und Vermessung von Strukturen.

Dieses Verfahren, das ohne ionisierende Strahlung auskommt, wird besonders zur Darstellung von Weichteilen und Knorpelstrukturen verwendet (Pasler 2008). Die Bilder des MRTs haben im Vergleich zum CT einen deutlich höheren Weichteilkontrast (Schwenzer und Ehrenfeld 2000). Von der Untersuchung ausgeschlossen werden müssen allerdings Risikopatienten, d. h. Patienten mit Herzschrittmacher, ferromagnetischen Metallteilen oder elektromechanischen Implantaten (Vogl 1991). Im Mund-, Kiefer- und Gesichtsbereich muss Außerdem berücksichtigt werden, dass metallische Klammern, Kronen oder Implantate das Magnetfeld stören können und somit eine Signalstörung bewirken (Wein et al. 1990).

Im Vergleich zum CT besteht technisch bedingt eine vergleichsweise deutlich längere Untersuchungsdauer (je nach Untersuchungsprotokoll etwa 30-40 min), sodass Bewegungsartefakte, oft durch Dyspnoe bedingt, eine teilweise deutliche Einschränkung der Beurteilbarkeit mit sich bringen (Becker 1998), obwohl die Aufnahmezeit pro Bild 0,8 Sekunden beträgt (Baik et al. 2002).

\subsection{Ziele der Arbeit}

Auf der Basis vorhandener DVT-Datensätze soll der Luftweg im Bereich des orofazialen Systems vermessen werden. Die dabei gemessenen Parameter dienen der Erstellung von Normwerten in horizontaler Körperposition mit Schwerpunkt der Ermittlung geschlechtsspezifischer Unterschiede.

Des Weiteren sollen die Zusammenhänge anatomischer Faktoren des aufsteigenden Unterkieferastes und der Luftwegsdimension dargestellt werden. Im Einzelnen sollte ermittelt werden:

1. der Luftweg und benachbarte Strukturen im Geschlechtervergleich,

2. Korrelation des kollapsfähigen Segmentes mit ausgewählten Parametern,

3 Korrelation der Luftwegsquerschnitte auf unterschiedlichen anatomischen Ebenen,

4. Korrelation der sagittalen und transversalen Dimensionen des Luftweges und

5 Neigung des Unterkieferastes zur mediosagittalen Ebene und Korrelation zum

Luftwegsquerschnitt. 


\section{Material und Methode}

\subsection{Patientenkollektiv}

Das Patientenkollektiv umfasste 129 Patienten im Alterskorridor von 30 bis 68 Jahre. Deren mittleres Alter betrug 45,73 Jahre \pm 11,09. Die Geschlechtseinteilung ergab, dass 64 Männer und 65 Frauen im Kollektiv betrachtet wurden. Das Durchschnittsalter der Männer betrug 45,8 3 Jahre $\pm 11,16$, das der Frauen 45,63 Jahre $\pm 11,12$.

Aus den ab 2006 bis Anfang 2009 vorhandenen DVT-Aufnahmen wurden konsekutiv 129 Datensätze mit vollständig dargestelltem Pharynx, Unterkiefer und Gaumen ausgewählt. Die Indikationen für diese Aufnahmen waren vor allem die prächirurgischen Abklärungen bei Zysten, Kieferhöhlenveränderungen, Weisheitszahnextraktionen oder zur präimplantorische Planung.

\subsection{Digitaler Volumentomograph NewTom OR-9000@}

Bei dem verwendeten Digitalen Volumentomographen NewTom QR-DVT 9000@ (Quantitative Radiology, Verona, Italien) (Abb.7) besteht der Detektor aus einem Bildverstärker (BV) der Firma Thomson mit einem BV-Eingangsfenster von 8x8 Zoll und einem Verstärkungsfaktor von 22:1. Die Bilderfassung erfolgte mit Hilfe eines $\mathrm{CCD}(\mathrm{CCD}=$ charge-couple-device) Chips mit einer Matrix von 512 x 512 Pixel. Die Aufnahme des bis zu 94 Megabyte großen Rohdatensatzes wurde mit der Software New Tom $9000 @$ Dental Version 3.37 bearbeitet.

Nach einem lateralen und anterior-posterioren Probescan berechnete das Programm die Hartgewebsdichtewerte und die Einstellparameter für die Belichtungsautomatik. Die erreichbare Dosisreduktion wurde durch die sogenannte ,smart-beam-Technik“ erzielt. Der Vorzug dieser Technik besteht darin, dass hierbei durch gezielte Analyse und Optimierung ausschließlich die kleinste ausreichende Strahlenexposition zur adäquaten Durchleuchtung des Objektes verwendet wird. Der Röhrenstrom variierte je nach Knochenvolumen und -dichte (Mozzo et al. 1998) und lag zwischen 0,5 und $10 \mathrm{~mA}$ (Holberg et al. 2005). Die Röntgenröhre arbeitete mit mittelharter Strahlung, d.h. es wurde eine konstante Spannung von $110 \mathrm{kV}$ verwendet. Die Filterung erfolgte mit 7,2 mm Aluminium bei einem konstanten Öffnungswinkel von 14 Grad. Bei der Aufnahme wurde ein Zylinder von $10 \mathrm{~cm}$ in der Höhe und $12 \mathrm{~cm}$ im Durchmesser abgebildet. Die gesamte Aufnahmezeit betrug 76 Sekunden, die tatsächliche Zeit der Strahlenexposition aufgrund des gepulsten Röntgenstrahls nur 18 Sekunden. Das mAs-Produkt lag, gesteuert über die 
Belichtungsautomatik, durchschnittlich zwischen 70 und 150 mAs (Holberg et al. 2005). Das akquirierte anatomische Volumen hatte eine Zylinderform mit den Abmessungen $15 \times 15 \mathrm{~cm}$.

Anschließend wurde aus den 360 einzelnen Projektionsradiographien ein dreidimensionaler Datensatz rekonstruiert. Die laterale Röntgenansicht ermöglicht dem Behandler die Angulation und den Schichtabstand der Primärrekonstruktion zu bestimmen. Das versetzt ihn in die Lage, eine individuelle und optimale Diagnostik durchzuführen. Die Dicke der axialen Schicht variierte zwischen 0,3 mm, $1 \mathrm{~mm}$ und $2 \mathrm{~mm}$, der maximale Durchmesser zwischen $128 \mathrm{~mm}$ (small field) und $148 \mathrm{~mm}$ (large field). Mit dem $148 \mathrm{~mm}$ umfassenden Durchmesser der axialen Schnitte war es bei entsprechender Positionierung des Patienten möglich, den gesamten für die Zahn-, Mund- und Kieferheilkunde relevanten Bereich, darzustellen. Die Voxelgröße betrug 0,25 x 0,25 x 0,25 mm. Es ließen sich beliebig viele Primärrekonstruktionen aus einem Rohdatensatz erstellen, da dieser archiviert wurde. Aus der Primärrekonstruktion konnten Sekundärrekonstruktionen in sagittalen, koronalen, paraaxialen sowie in Panoramaschichten und 3D Ansichten erstellt werden (Mozzo et al. 1998, Moebes et al. 2000). Mit der verwendeten NewTom Software (Version 3.37) war es möglich, in den konstruierten Schnitten Strecken und Winkel zu messen sowie Hilfsmarker zu setzen. Es kamen 0,5 mm, 1,5 mm oder 3,0 $\mathrm{mm}$ große farbige Marker zur Verwendung. Die automatische Übertragung der Farbmarkierungen in allen Ebenen ermöglichte eine Vermessung von Distanzen im Raum. Die in diesem Programm angegebenen Werte entsprachen den anatomischen Strecken in mm. Zur Auswertung der Röntgenaufnahmen wurden die von den Herstellern gelieferte und empfohlene Software benutzt, welche eine metrische Genauigkeit von 0,1 mm besitzt (Hübinger 2008).

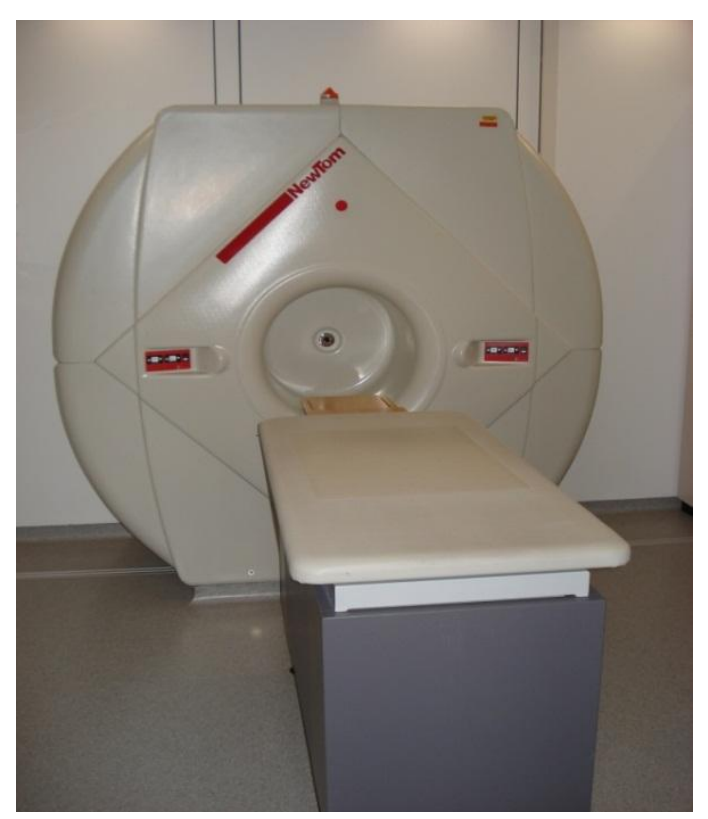

Abb. 7: Der Digitale Volumentomograph NewTom QR-DVT 9000@ (Quantitative Radiology, Verona, Italien) 


\subsection{Konstanzprüfung des DVT}

Die Eichung des DVT erfolgte im Rahmen der Konstanzprüfung. Die Überprüfung des Befundsmonitors fand täglich mit Hilfe des SMPTE-Testbildes statt, ebenso wie die des Digitalen Volumentomographen bei Inbetriebnahme. Dabei wurde der Testkörper mittels eines Lasers, entsprechend den Markierungen am Prüfkörper, in der Gantry ausgerichtet. Der Prüfkörper war ein Aluminiumrohr, welches auf 3, 6, 9 und 12 Uhr jeweils 2 Bohrungen enthielt. Ummantelt wurde dieses mittels eines Plexiglaszylinders. Der gesamte Prüfkörper war mit Wasser gefüllt, wobei keine Luftblasen eingeschlossen sein durften.

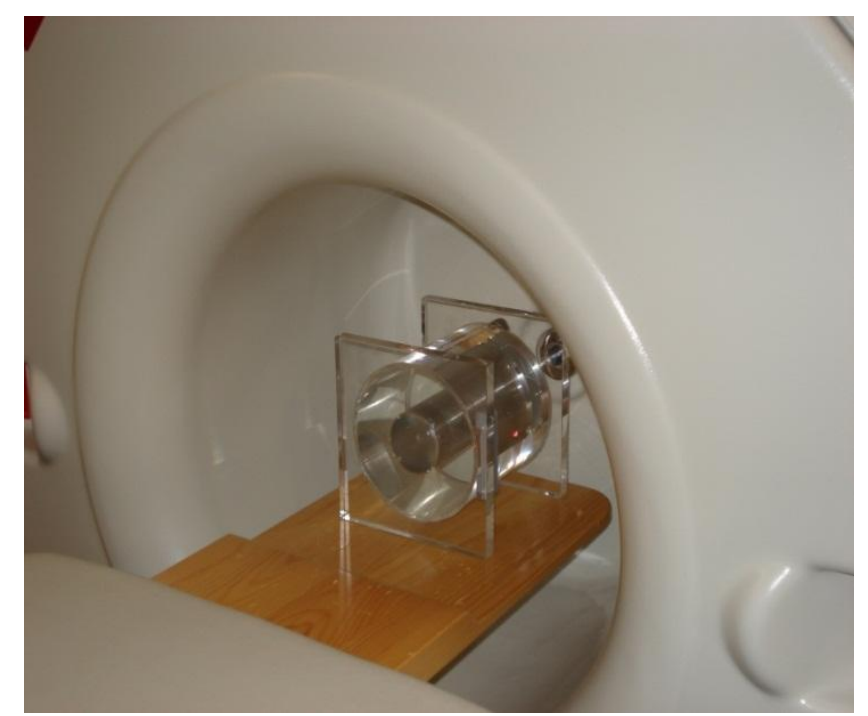

Abb. 8: Prüfkörper für die Konstanzprüfung des NewTom QR-DVT 9000@

Die vom Hersteller empfohlene monatliche Konstanzprüfung erfolgte von medizinisch technischen Angestellten der Universität Göttingen, wobei der gleiche Prüfkörper benutzt wurde (Abb. 8).

Folgende Arbeitsschritte wurden als Standard für die Verwendungsphase des Prüfkörpers festgelegt. Als Erstes wurde überprüft, ob das Nutzstrahlenfeld mit den Lasermarkierungen übereinstimmt, als Zweites die Hochkontrastauflösung mittels eines Bleistrichgitters, das auf der rechten Seite des Prüfkörpers befestigt wurde. Im $90^{\circ}$-Seitenbild musste mindestens 1 Linienpaar pro mm sichtbar sein.

Danach erfolgte die Kontrolle der Niedrigkontrastauflösung mit Hilfe der Kontrastunterschiede zwischen Wasser und Aluminium im Bereich der Bohrungen. Der Graustufenunterschied zwischen den Maximal- und Minimalwerten musste größer als 35 Graustufen sein.

Anschließend wurde die Abbildungsgeometrie in der Panoramarekonstruktion gemessen. Der gemessene vertikale Abstand der Bohrungen des Prüfkörpers durfte nicht mehr als $1 \mathrm{~mm}$ von einem vom Programm ermittelten Wert abweichen. Das in der Primärrekonstruktion des Prüfkörpers 
gemessene Bildelementerauschen musste im Bereich von 0-5\% liegen. Nur die in der Abnahmeprüfung angegebene Artefakte durften in der Aufnahme mit dem Prüfkörper sichtbar sein.

\subsection{DVT-Softwareeinstellungen}

Die Aufnahmen wurden in der Universitätsklinik Göttingen, Abteilung Mund-, Kiefer- und Gesichtschirurgie, mit dem Digitalen Volumentomographen der Firma NewTom (Marburg), Modell QR-DVT 9000@ erstellt.

Das Röntgen der Patienten erfolgte im wachen und auf dem Rücken liegenden Zustand, mit geschlossenem Mund, ruhig durch die Nase atmend und mit habitueller Interkuspitation.

Die Datensätze wertete ich an dem verwendeten Befundsmonitor KAT.B nach DIN 6868-57 U. QSRL des Herstellers EIZO und der NewTom Software Version 3.37 aus.

Pro Untersuchung wurde rechnergestützt ein dreidimensionaler Datensatz rekonstruiert. Die Ausrichtung der primär axialen Rekonstruktion erfolgte parallel der harten Gaumenebene (PNSANS) und erstreckte sich von oberhalb des harten Gaumens bis zum Hyoid. Folgende Einstellungsparameter wurden als Standard für die Primärrekonstruktion definiert: „Large field“ und $1 \mathrm{~mm}$ dicke axiale Schnitte.

\subsection{Vermessung der DVT-Datensätze}

Um das orofaziale System zu vermessen und die Ergebnisse mit anderen Studien (Kapitel 4.3.) zu vergleichen wurde auf Messpunkte und Messstrecken, die in den folgenden Abbildungen (Abb. 9Abb. 13) beschrieben werden zurückgegriffen. Dabei handeln es sich vor allem um Messpunkte, die in der OSAS-Diagnostik Verwendung finden (Tabelle 1, Kapitel 1.3.2. und Kapitel 4.1.4.). Zur Erstellung einer aussagefähigen Datenmatrix wurden für die Erstellung der Sekundärrekonstruktion folgende Schnitte und Parameter ausgewählt. Ein mediosagittaler Schnitt orientiert an ANS und PNS und ein koronaler Schnitt auf der Hälfte der ANS-PNS-Strecke.

In dem mediosagittalen Schnitt (Abb. 9 und Abb. 10) wurden die Länge des harten Gaumens (ANSPNS), die Weichgaumenlängee (PNS-Uvulaspitze), die Weichgaumenbreite an der breitesten Stelle, die Kontaktstrecke von der Zunge zum weichen Gaumen, die Höhe des kollapsfähigen Segmentes (Epiglottisspitze-PNS), der kürzeste Abstand vom Hyoidsenkrecht zur Nasallinie (NL), die Strecke zwischen ANS und dem Schnittpunkt der Nasallinie und Hyoidsenkrechten sowie der subpalatinale Luftraum gemessen. Die beiden folgenden Tabellen zeigen einen Teil der Messpunkte und strecken. 
Tabelle 2: Auflistung der Messpunkte. In der Tabelle sind die Messpunkte aufgelistet, die in den folgenden Abbildungen dargestellt sind.

\begin{tabular}{|c|c|}
\hline Messpunkt & \\
\hline 1 & Vallecula glossoepiglottica \\
\hline 2 & Dorsalster Punkt des Zungenrückens \\
\hline 3 & Vordere Begrenzung des Luftweges auf Höhe des unteren Inzisialpunktes \\
\hline 4 & Anteriorer Punkt des Isthmus \\
\hline 5 & Spina nasalis anterior (ANS) \\
\hline 6 & Spina nasalis posterior (PNS) \\
\hline 7 & Kaudale Begrenzung der Kontaktfläche der Zunge mit dem weichen Gaumen \\
\hline $77^{\prime}$ & Kraniale Begrenzung der Kontaktfläche der Zunge mit dem weichen Gaumen \\
\hline 8 & Epiglottisspitze \\
\hline 9 & Uvulaspitze \\
\hline 10 & Punkt auf der oralen Fläche, an dem der weiche Gaumen am breitesten ist \\
\hline $10^{\prime}$ & Punkt auf der nasalen Fläche, an dem der weiche Gaumen am breitesten ist \\
\hline 11 & Schnittpunkt der Hyoidsenkrechten und der Nasallinie \\
\hline 12 & Vorderster oberster Punkt des Hyoids \\
\hline 13 & Begrenzung des lufthaltigen Raumes durch die Hartgaumenstruktur \\
\hline $13^{\prime}$ & Begrenzung des lufthaltigen Raumes durch die Zungenstruktur \\
\hline 14 & Linguale Höckerspitze des rechten unteren Sechsjahresmolaren \\
\hline 15 & Linguale Höckerspitze des linken unteren Sechsjahresmolaren \\
\hline 16 & Harter Gaumen in der Mediosagittalen \\
\hline 17 & Schnittpunkt der Mediosagittalen mit der Verbindungslinie zwischen den lingualen Höckerspitzen der unteren Sechsjahresmolaren \\
\hline 18 & Schnittpunkt der Mediosagittalen mit der Verbindungslinie der Unterkieferspange unter Begrenzung der unteren Kontur des Unterkiefers \\
\hline 19 & Unterer Inzisialpunkt \\
\hline 20 & Hintere Pharynxwand auf Höhe des unteren Inzisialpunktes \\
\hline 21 & Vordere Begrenzung des Luftweges auf Höhe des unteren Inzisialpunktes \\
\hline 22 & Transversale Begrenzung des Luftweges auf Höhe des unteren Inzisialpunktes links \\
\hline 23 & Transversale Begrenzung des Luftweges auf Höhe des unteren Inzisialpunktes rechts \\
\hline 26 & Schnittpunkt Isthmussenkrechten mit der Nasallinie \\
\hline$\alpha$ & Winkel zwischen der Innenkortikalis der Vorderkante des aufsteigenden Unterkieferastes und der Verbindungslinie der distalen Konturen des Unterkiefers \\
\hline$\beta$ & Winkel zwischen der Außenkortikalis der Vorderkante des aufsteigenden Unterkieferastes und der Verbindungslinie der distalen Konturen des Unterkiefers \\
\hline
\end{tabular}

Tabelle 3: Auflistung der Messstrecken. In der Tabelle sind die Messstrecken aufgelistet, die in den folgenden Abbildungen dargestellt sind.

\begin{tabular}{|l|l|}
\hline Strecken & \\
\hline $4-26$ & Abstand des Isthmus zur Nasallimie (Isthmus-PNS)(Isthmus-PNS) \\
\hline $5-6$ & Länge harter Gaumen (ANS-PNS) \\
\hline $5-8$ & Höhe des kollapsfähigen Segmentes (PNS-Epiglottisspitze) \\
\hline $5-9$ & Weichgaumenlänge \\
\hline $6-11$ & Abstand zwischen Spina nasalis anterior und dem Schnittpunkt der Nasallinie und der Hyoidsenkrechten (ANS-Hyoidsenkrechten) \\
\hline $7-7^{\prime}$ & Kontaktstrecke weicher Gaumen mit Zunge \\
\hline $10-10^{\prime}$ & Weichgaumenbreite an der breitesten Stelle lotrecht zur Strecke 7-7' \\
\hline $11-12$ & kürzester Abstand vom Hyoid senkrecht zur Nasallinie (Hyoid-Nasallinie) \\
\hline $13-13$ & maximaler Luftraum zwischen harten Gaumen und Zunge =Subpalatinaler Luftraum \\
\hline $14-15$ & Abstand der lingualen Höckerspitzen der unteren Sechsjahresmolaren \\
\hline $16-17$ & Abstand der Nasallinie zur Verbindungslinie zwischen den lingualen Höckerspitzen der unteren Sechsjahresmolaren=palatinale Höhe \\
\hline $16-18:$ & Abstand der Nasallinie zur Verbindungslinie der Unterkieferspange (NL-UK) \\
\hline $19-20$ & Intermaxilläre Raumlänge \\
\hline $20-21$ & Sagittale Ausdehnung des Luftweges auf Höhe des unteren Inzisialpunktes (MPW) \\
\hline $22-23$ & Transversale Ausdehnung des Luftweges auf Höhe des unteren Inzisialpunktes (MPW) \\
\hline
\end{tabular}




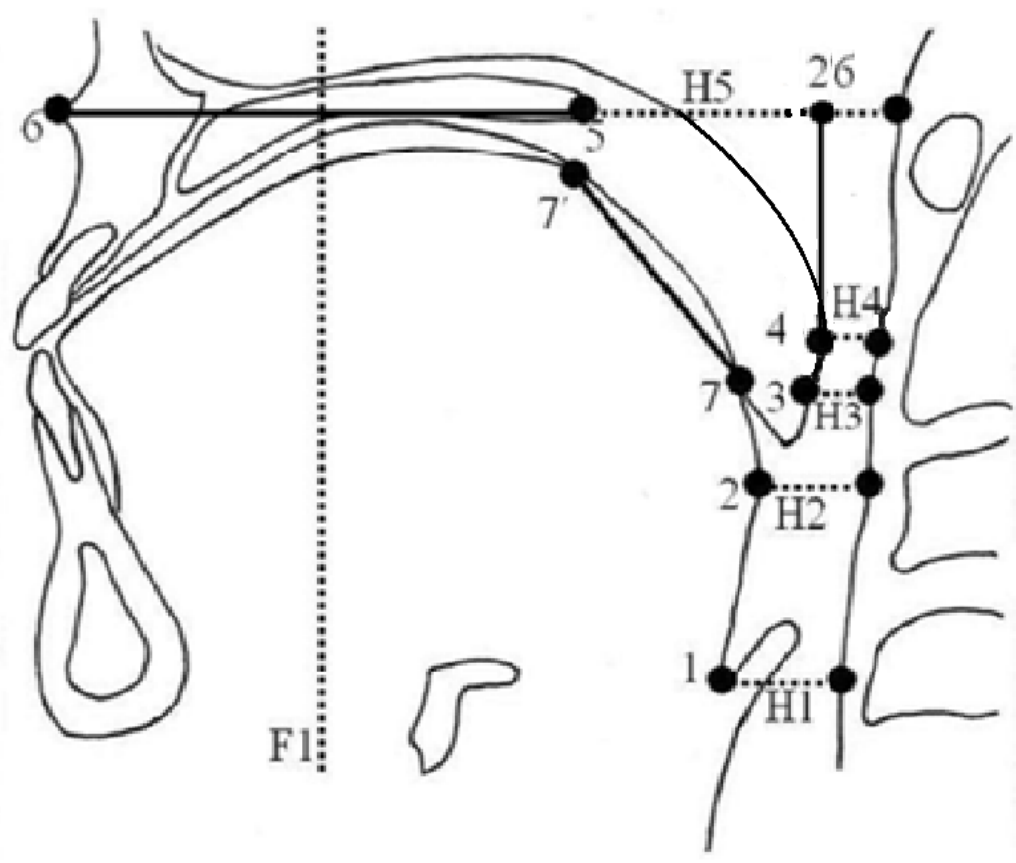

Abb. 9: Schnitt in der Mediosagittalen (modifiziert nach Markiewicz 2011)

1. Vallecula glossoepiglottica

2. Dorsalster Punkt des Zungenrückens

3. Vordere Begrenzung des Luftweges auf Höhe des unteren Inzisialpunktes

4. Anteriorer Punkt des Isthmus

5. Spina nasalis posterior (PNS)

6. Spina nasalis anterior (ANS)

7. Kaudale Begrenzung der Kontaktfläche der Zunge mit dem weichen Gaumen

7'. Kraniale Begrenzung der Kontaktfläche der Zunge mit dem weichen Gaumen

26. Schnittpunkt Isthmussenkrechten mit der Nasallinie

Strecke 5-6: Länge harter Gaumen (ANS-PNS)

Strecke 7-7': Kontaktstrecke weicher Gaumen mit Zunge

Strecke 4-26: Abstand des Isthmus zur Nasallimie (Isthmus-PNS)

F1: Koronale Schnittebene senkrecht auf Höhe der Hälfte des Abstandes zwischen Spina nasalis anterior und Spina nasalis posterior

H1: Axiale Schnittebene auf Höhe der Vallecula glossoepiglottica parallel zur Nasallinie (Lower Pharyngeal Wall (LPW))

H2: Axiale Schnittebene auf Höhe des dorsalsten Punktes des Zungenrückens parallel zur Nasallinie (Tongue Pharyngeal Wall (TPW))

H3: Axiale Schnittebene auf Höhe des unteren Inzisialpunktes parallel zur Nasallinie (Middle Pharyngeal Wall (MPW))

H4: Axiale Schnittebene auf Höhe des Isthmus parallel zur Nasallinie (Isthmus)

H5: Axiale Schnittebene auf Höhe der Nasallinie (Upper Pharyngeal Wall (UPW)) 


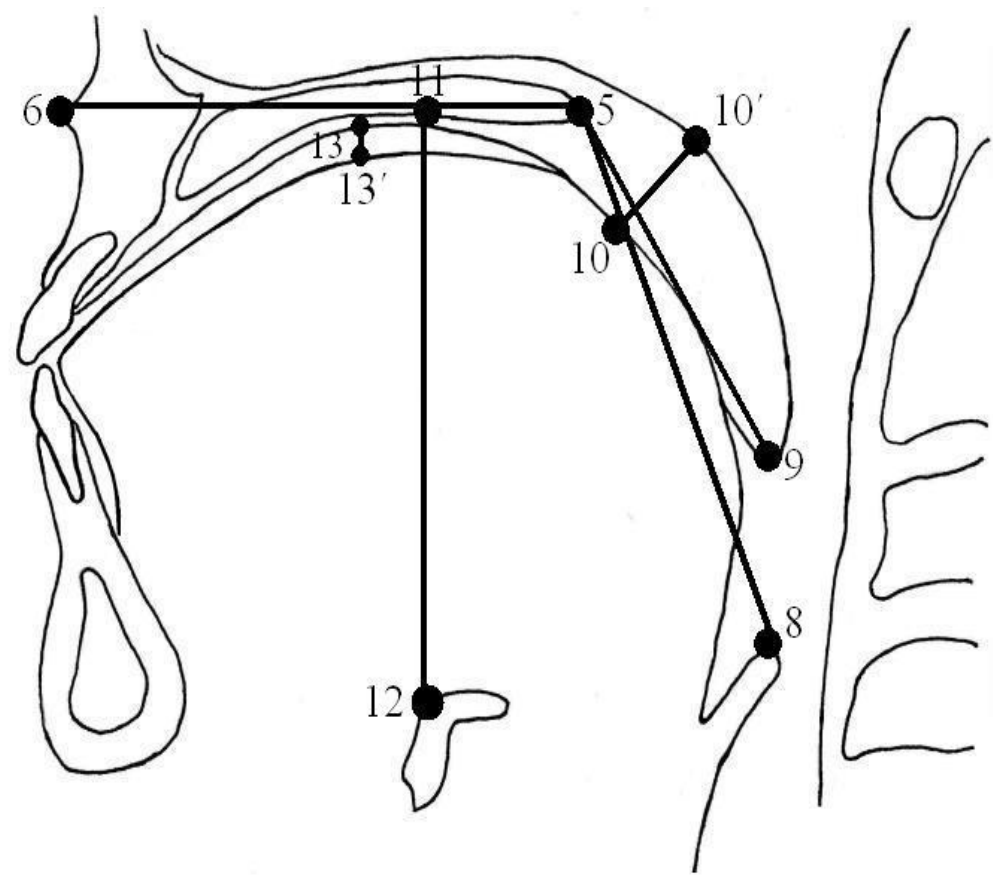

Abb. 10: Schnitt in der Mediosagittalen (nach Markiewicz 2011)

5. Spina nasalis posterior (PNS)

6. Spina nasalis anterior (ANS)

8. Epiglottisspitze

9. Uvulaspitze

10. Punkt auf der oralen Fläche, an dem der weiche Gaumen am breitesten ist

10'. Punkt auf der nasalen Fläche, an dem der weiche Gaumen am breitesten ist

11. Schnittpunkt der Hyoidsenkrechten und der Nasallinie

12. Vorderster oberster Punkt des Hyoids

13. Begrenzung des lufthaltigen Raumes durch die Hartgaumenstruktur

13'. Begrenzung des lufthaltigen Raumes durch die Zungenstruktur

Strecke 5-8: Höhe des kollapsfähigen Segmentes (PNS-Epiglottisspitze)

Strecke 5-9: Weichgaumenlänge

Strecke 6-11: Abstand zwischen Spina nasalis anterior und dem Schnittpunkt der Nasallinie und der Hyoidsenkrechten (ANS-Hyoidsenkrechten)

Strecke 10-10': Weichgaumenbreite an der breitesten Stelle lotrecht zur Strecke 7-7'

Strecke 11-12: kürzester Abstand vom Hyoid senkrecht zur Nasallinie (Hyoid-Nasallinie)

Strecke 13-13': maximaler Luftraum zwischen harten Gaumen und Zunge =Subpalatinaler Luftraum 
Im koronalen Schnitt (Abb. 11) wurde die Strecke zwischen dem harten Gaumen und der Verbindungslinie an der Unterseite der rechten und linken Unterkieferspange, die palatinale Höhe sowie der Abstand zwischen den lingualen Höckern der Unterkieferzähne gemessen.

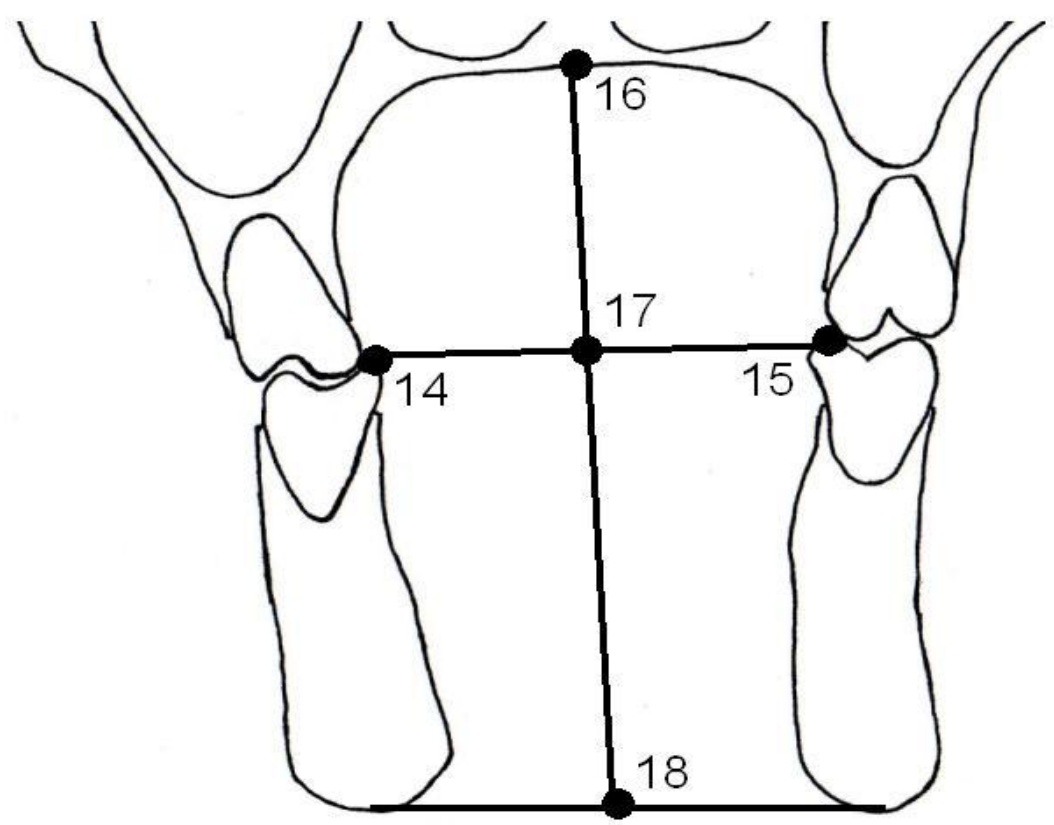

Abb. 11: Koronaler Schnitt auf der Hälfte der Nasallinie (nach Markiewicz 2011)

14. Linguale Höckerspitze des rechten unteren Sechsjahresmolaren

15. Linguale Höckerspitze des linken unteren Sechsjahresmolaren

16. Harter Gaumen in der Mediosagittalen

17. Schnittpunkt der Mediosagittalen mit der Verbindungslinie zwischen den lingualen Höckerspitzen der unteren Sechsjahresmolaren

18. Schnittpunkt der Mediosagittalen mit der Verbindungslinie der Unterkieferspange unter Begrenzung der unteren Kontur des Unterkiefers

Strecke 14-15: Abstand der lingualen Höckerspitzen der unteren Sechsjahresmolaren

Strecke 16-17: Abstand der Nasallinie zur Verbindungslinie zwischen den lingualen Höckerspitzen der unteren Sechsjahresmolaren=palatinale Höhe

Strecke 16-18: Abstand der Nasallinie zur Verbindungslinie der Unterkieferspange (NL-UK) 
In den axialen Schnitten, die alle parallel zur harten Gaumenebene ausgerichtet wurden, erfolgte die Vermessung des Luftweges auf Höhe von PNS (Upper Pharyngeal Wall (UPW)), der Vallecula glossoepiglottica (Lower Pharyngeal Wall (LPW)), des dorsalsten Punktes der Zunge (Tongue Pharyngeal Wall (TPW)), des unteren Inzisialpunktes (Middle Pharyngeal Wall (MPW)) (Abb. 12) und der schmalsten Stelle des Luftweges (Isthmus) in transversaler und sagittaler Richtung im Bereich der größten Ausdehnung. Besonderes Augenmerk wurde darauf gelegt, dass die transversalen und sagittalen Verbindungsstrecken senkrecht zueinander orientiert waren. Der Ort des Isthmus wurde als die Stelle definiert, welche bei der Messung in der mediosagittalen Ebene die geringste Ausdehnung in der anterior-posterioren Richtung hatte. Außerdem wurde der Isthmus folgendermaßen bestimmt, in dem in den axialen Schnitten der kleinste anterior-posteriore Durchmesser ausgewählt wurde. Weiterhin wurden auf Höhe des unteren Inzisialpunktes die intermaxilläre Raumlänge (Unterkieferinzisivi zur dorsalen Pharynxwand) und die Winkel zwischen der Innenkortikalis (Winkel $\alpha$ ) bzw. der Außenkortikalis (Winkel $\beta$ ) des linken aufsteigenden Unterkieferastes und der Verbindungslinie der distalen Konturen des Unterkiefers vermessen. Bei zweigeteilten Luftwegen erfolgte die Vermessung der beiden Öffnungsquerschnitte separat nach den oben beschriebenen Kriterien. Die Fläche des Luftweges wurde als Ellipse angenommen und mit der Formel $\mathrm{A}=\pi^{*} 1 / 2 \mathrm{a}^{*} 1 / 2 \mathrm{~b}$ berechnet.

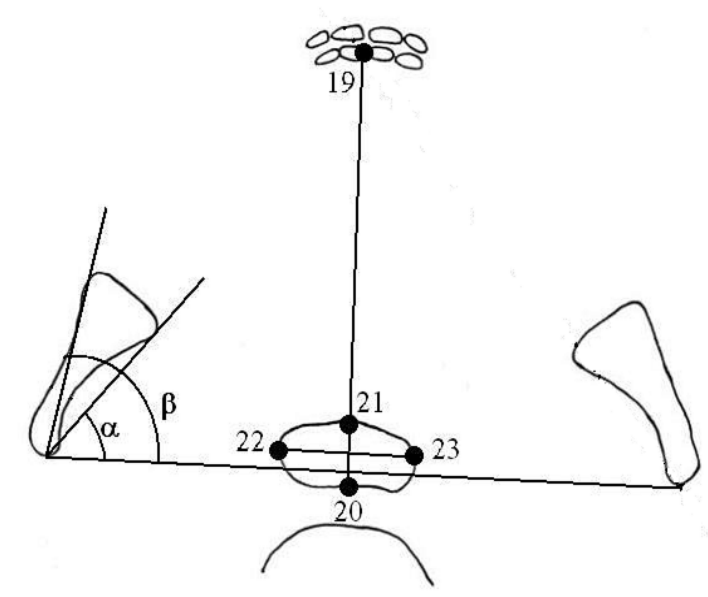

Abb. 12: Axialer Schnitt auf Höhe des unteren Inzisialpunktes (MPW) (nach Markiewicz 2011)

19. Unterer Inzisialpunkt

20. Hintere Pharynxwand auf Höhe des unteren Inzisialpunktes

21. Vordere Begrenzung des Luftweges auf Höhe des unteren Inzisialpunktes

22. Transversale Begrenzung des Luftweges auf Höhe des unteren Inzisialpunktes links

23. Transversale Begrenzung des Luftweges auf Höhe des unteren Inzisialpunktes rechts

$\alpha$. Winkel zwischen der Innenkortikalis der Vorderkante des aufsteigenden Unterkieferastes und der Verbindungslinie der distalen Konturen des Unterkiefers

$\beta$. Winkel zwischen Außenkortikalis der Vorderkante des aufsteigenden Unterkieferastes und der

Verbindungslinie der distalen Konturen des Unterkiefers

Strecke 19-20: Intermaxilläre Raumlänge

Strecke 20-21: Sagittale Ausdehnung des Luftweges auf Höhe des unteren Inzisialpunktes

Strecke 22-23: Transversale Ausdehnung des Luftweges auf Höhe des unteren Inzisialpunktes 


\subsection{Statistische Auswertung}

Die statistische Auswertung der vorliegenden Daten erfolgte mit dem Programm Statistica V 8 (Firma Statsoft, Tulsa, USA).

Der Vergleich der metrischen Werte erfolgte mit Hilfe von Korrelationsmatrizen nach Pearson paarweise. Die Gegenüberstellung zweier unabhängiger Stichproben (Vergleich Geschlecht mit metrischen Werten) wurde mit dem Mann-Whitney-U-Test durchgeführt.

Das Signifikanzniveau betrug $\alpha=5 \%$. Damit waren alle Ergebnisse mit einem $p$-Wert kleiner als 0,05 signifikant und wurden mit rot markiert. Die p-Werte wurden bis zur dritten Stelle nach dem Komma angegeben. Alle Ergebnisse ab p>0,001 wurden mit ihren exakten Werten angegeben.

Zur graphischen Darstellung der Werte wurden Box-Plots und Histogramme benutzt.

Für jeden Messparameter, auf die Y-Achse aufgetragen, wurde ein Box-Plot erstellt, unterteilt in Männer und Frauen (X-Achse). Für die Box nutzte man die einfache Standardabweichung, für den Whisker die Minimum-Maximumwerte. Zusätzlich wurden den Mittelwert als Punkt innerhalb der Box dargestellt. Bei den Histogrammen wurden die Messwerte in Abszissenrichtung und die Häufigkeiten in Ordinatenrichtung aufgetragen sowie Männer und Frauen unterschiedlich dargestellt (Männer blau, Frauen rot), um Unterschiede zwischen den Geschlechtern aufzuzeigen.

\subsection{Ethikkommissionsvotum}

Die Ethikkommission der Medizinischen Fakultät hatte hinsichtlich der Durchführung dieser Untersuchung keine Bedenken (Antragsnummer: 7/6/08). 


\section{Ergebnisse}

\subsection{Luftweg und benachbarte Strukturen im Geschlechtervergleich}

\subsubsection{Mediosagittale Messungen}
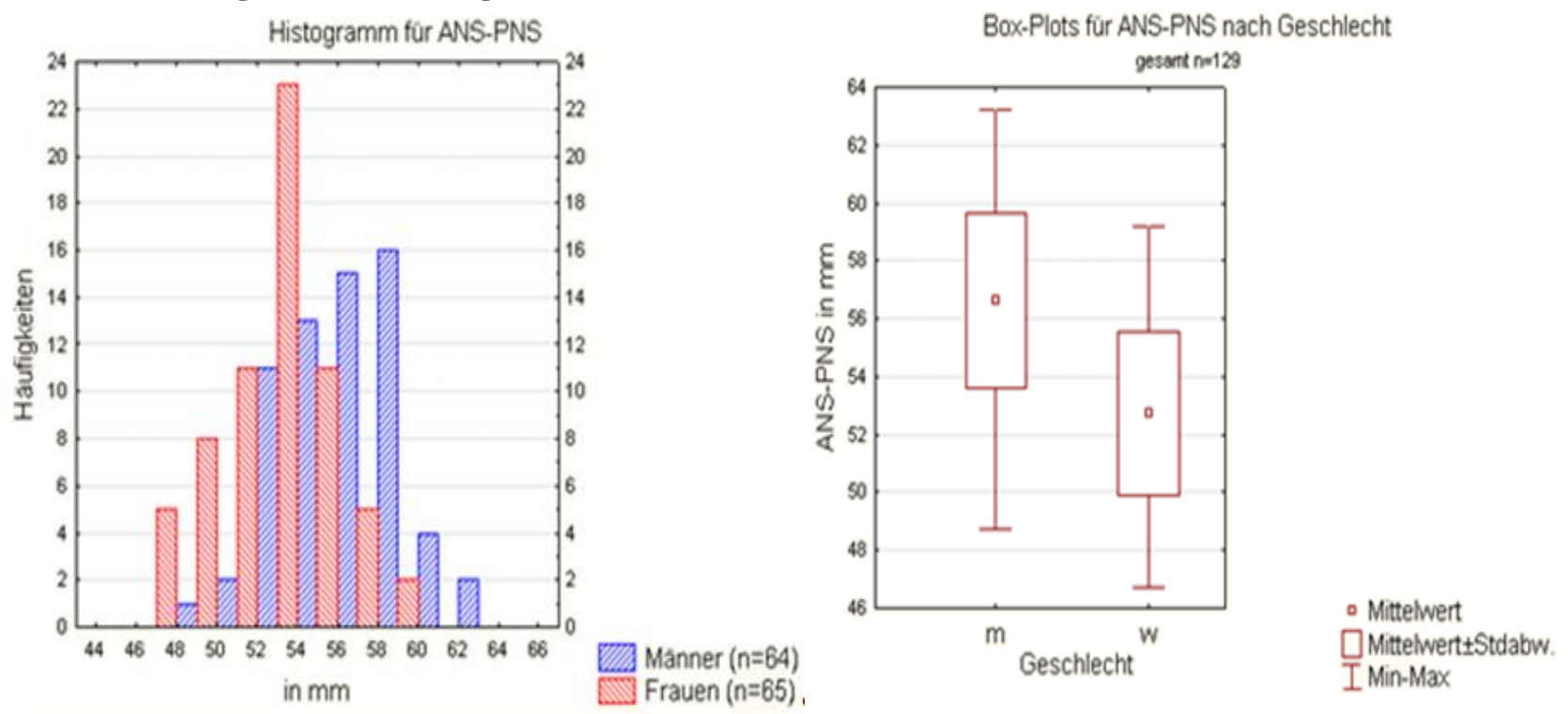

Abb. 13: Box-Plots und Histogramme für die Strecke ANS-PNS

Der Mittelwert für die Strecke ANS-PNS betrug für die Männer 56,64 mm \pm 3,02 und für die Frauen 52,72 $\mathrm{mm} \pm 2,82$. Der Vergleich (Mann-Whitney-U-Test) beider Kollektive ergab einen $\mathrm{p}$ Wert $<0,001$, somit bestand ein signifikanter Unterschied zwischen beiden Kollektiven. Männer weisen eine größere Strecke ANS-PNS als Frauen auf
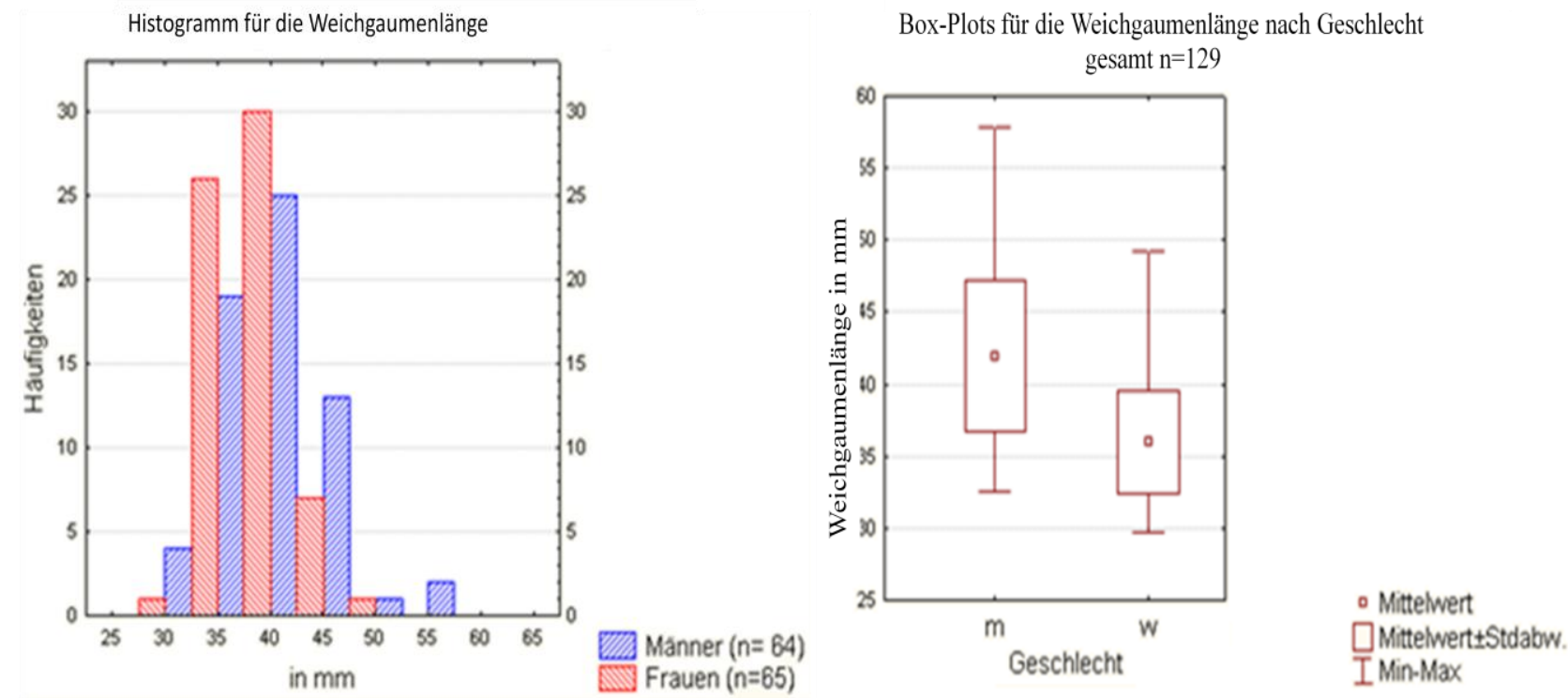

Abb. 14: Box-Plots und Histogramme für die Weichgaumenlänge

Der Mittelwert für die Weichgaumenlänge betrug für Männer 41,93 mm \pm 5,24 und für Frauen 35,99 $\mathrm{mm} \pm 3,61$. Der Vergleich beider Kollektive ergab einen $\mathrm{p}$-Wert <0,001, somit bestand ein 


\section{Ergebnisse}

signifikanter Unterschied zwischen beiden Kollektiven, dabei hatten Männer einen längeren weichen Gaumen als Frauen.
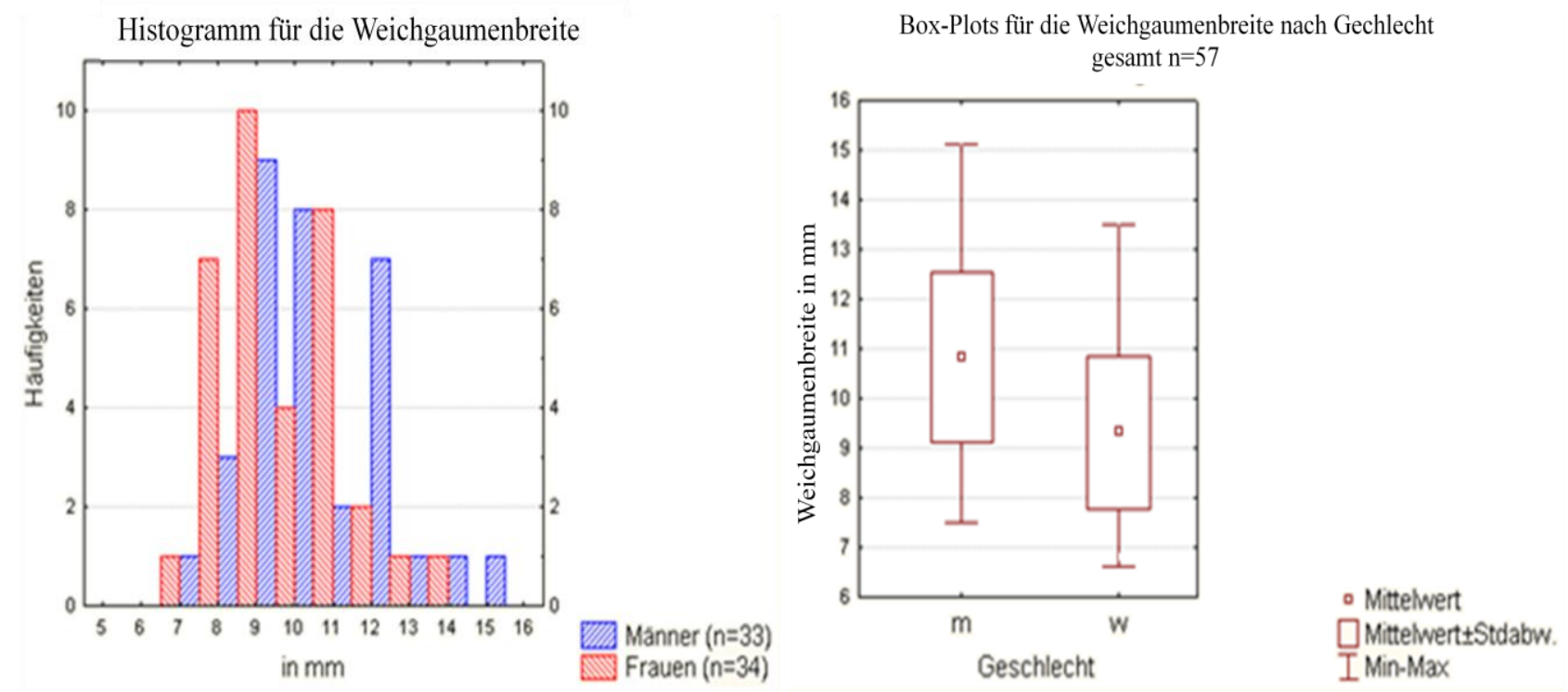

Abb. 15: Box-Plots und Histogramme für die Weichgaumenbreite

Der Mittelwert für die Weichgaumenbreite betrug für Männer 10,83 mm \pm 1,71 und für Frauen 9,31 $\mathrm{mm} \pm 2,41$. Der Vergleich beider Kollektive ergab einen p-Wert <0,001, somit bestand ein signifikanter Unterschied zwischen beiden Kollektiven. Männer wiesen einen breiteren weichen Gaumen auf als Frauen.
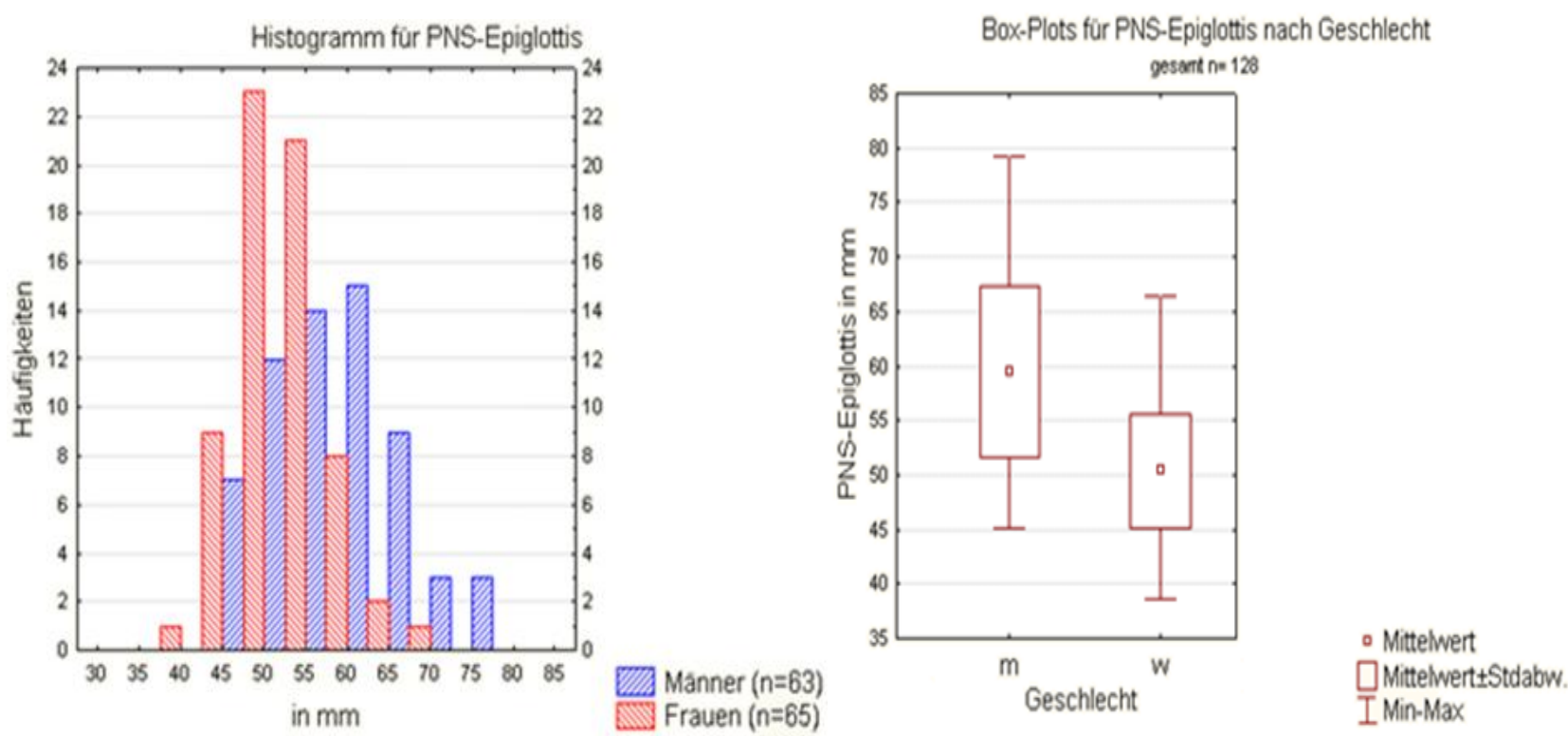

Abb. 16: Box-Plots und Histogramme für die Strecke PNS-Epiglottis

Der Mittelwert für die Strecke PNS-Epiglottis betrug für Männer 59,52 mm \pm 7,83 und für Frauen $50,38 \mathrm{~mm} \pm 5,27$. Der Vergleich beider Kollektive ergab einen $\mathrm{p}$-Wert <0,001, somit bestand ein 


\section{Ergebnisse}

signifikanter Unterschied zwischen beiden Kollektiven, dabei war die Strecke PNS-Epiglottis bei Männern größer als bei Frauen.
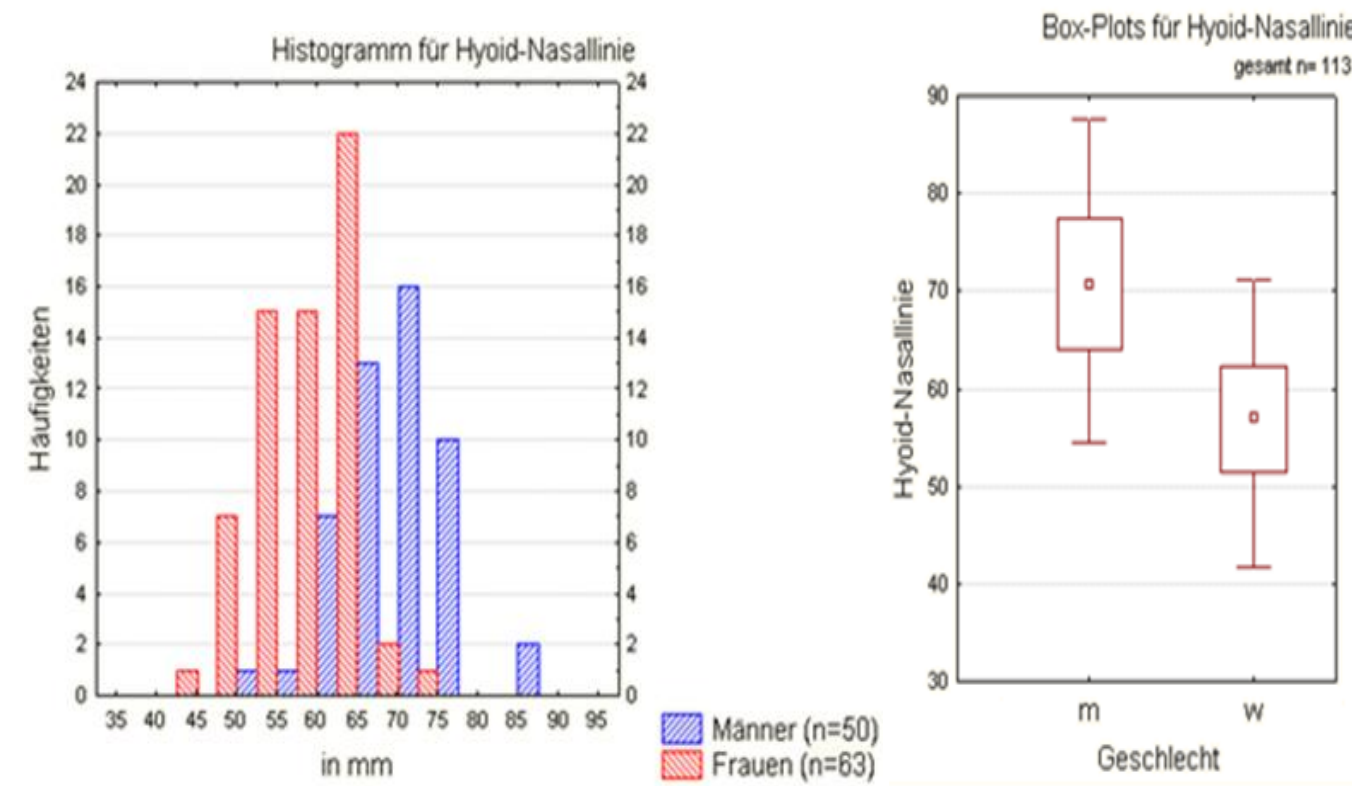

- Mittelwert
Mittelwert \pm Stdabw.

Abb. 17: Box-Plots und Histogramme für die Strecke Hyoid zur Nasallinie

Der Mittelwert für die Strecke Hyoid-Nasallinie betrug für Männer 70,63 mm \pm 6,71 und für Frauen 56,97 $\mathrm{mm} \pm 5,42$. Der Vergleich beider Kollektive ergab einen $\mathrm{p}$-Wert $<0,001$, somit bestand ein signifikanter Unterschied zwischen beiden Kollektiven, dabei wiesen Männer eine größere Strecke Hyoid-Nasallinie als Frauen auf.

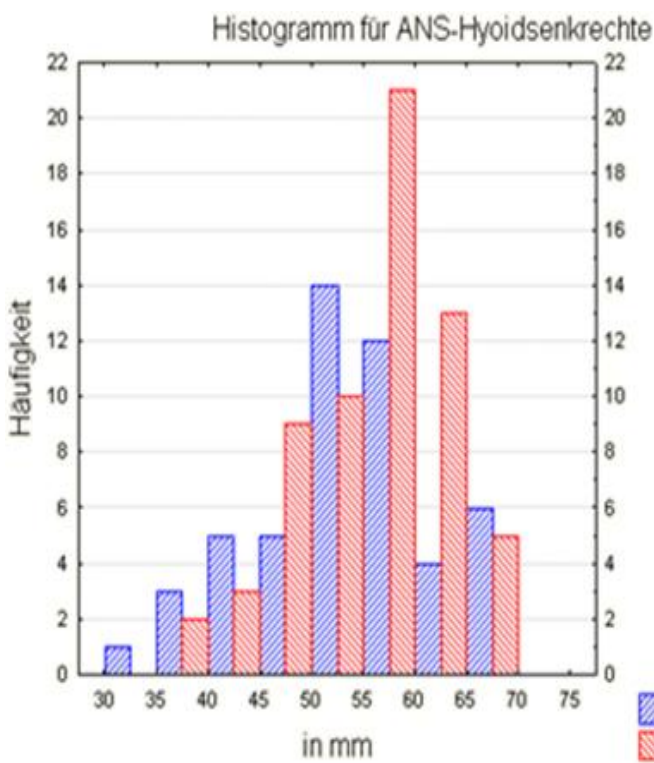

Mànner $(n=50)$

Frauen $(n=63)$

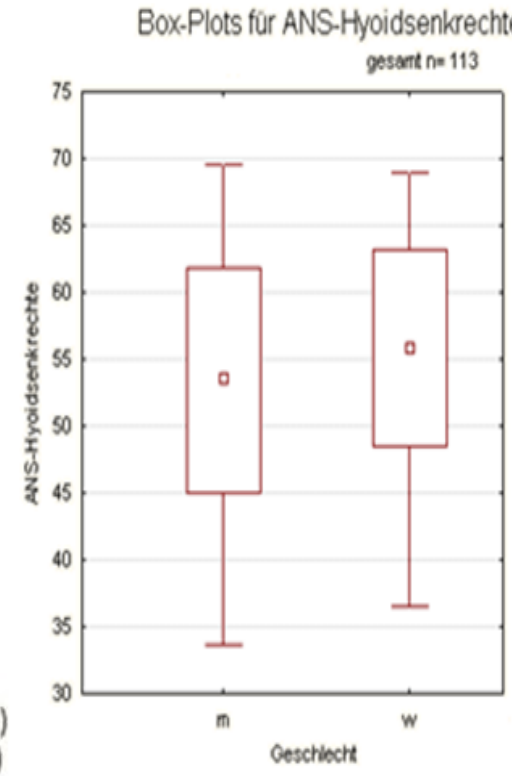

Abb.18: Box-Plots und Histogramme für die Strecke ANS-Hyoidsenkrechte

Der Mittelwert für die Strecke ANS-Hyoidsenkrechte betrug für Männer 53,46 mm \pm 8,40 und für Frauen 55,80 \pm 7,35. Der Vergleich beider Kollektive ergab einen p-Wert <0,001, somit bestand ein signifikanter Unterschied zwischen beiden Kollektiven. 


\section{Ergebnisse}
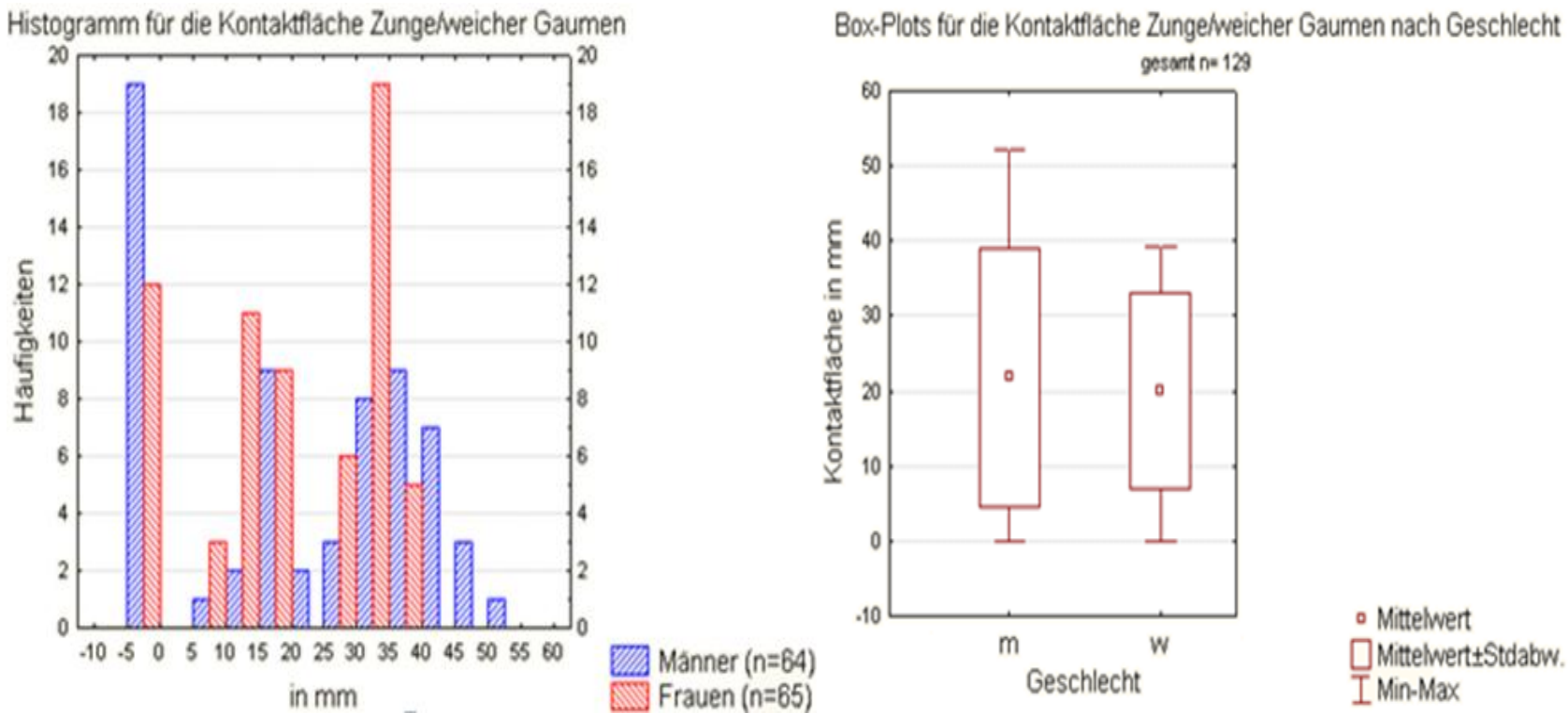

Abb. 19: Box-Plots und Histogramme für die Kontaktstrecke Zunge und weicher Gaumen

Der Mittelwert für die Kontaktstrecke zwischen dem weichen Gaumen und der Zunge betrug für die Männer 21,84 mm \pm 17,21 und für die Frauen 20,09 mm \pm 13,04. Der Vergleich beider Kollektive ergab einen p-Wert von 0,299, somit bestand kein signifikanter Unterschied zwischen beiden Kollektiven. Die Kontaktstrecken waren bei den Männern größer als bei Frauen.

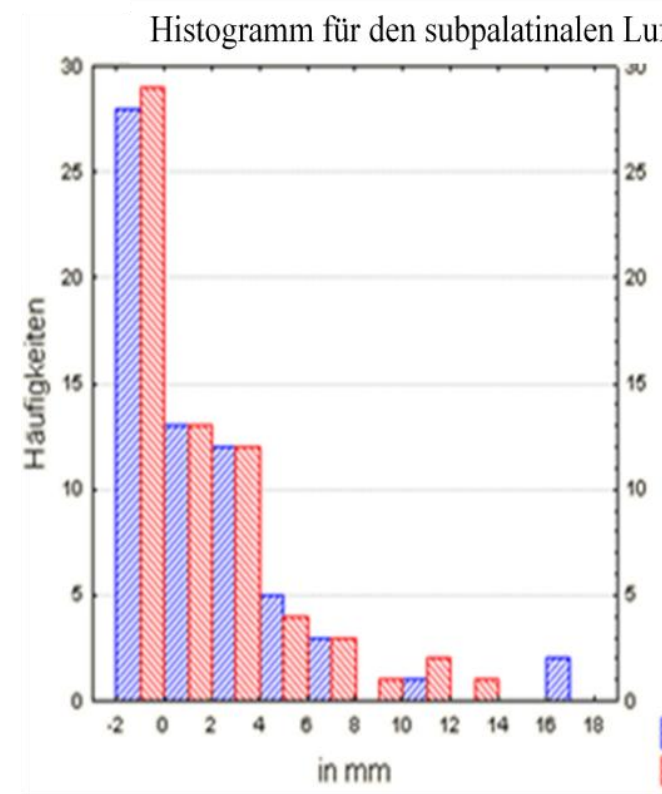

Box-Plots für den subpalatinalen Luftraum nach Geschlecht gesamt $n=129$

Abb. 20: Box-Plots und Histogramme für den subpalatinalen Luftraum

Der Mittelwert für den subpalatinalen Luftraum betrug für die Männer 2,23 mm \pm 3,40 und für die Frauen 2,08 $\mathrm{mm} \pm 2,95$. Der p-Wert beider Kollektive lag bei 0,841, somit bestand kein signifikanter Unterschied zwischen beiden Kollektiven. Der subpalatinle Luftraum war bei den Männern leicht größer. 

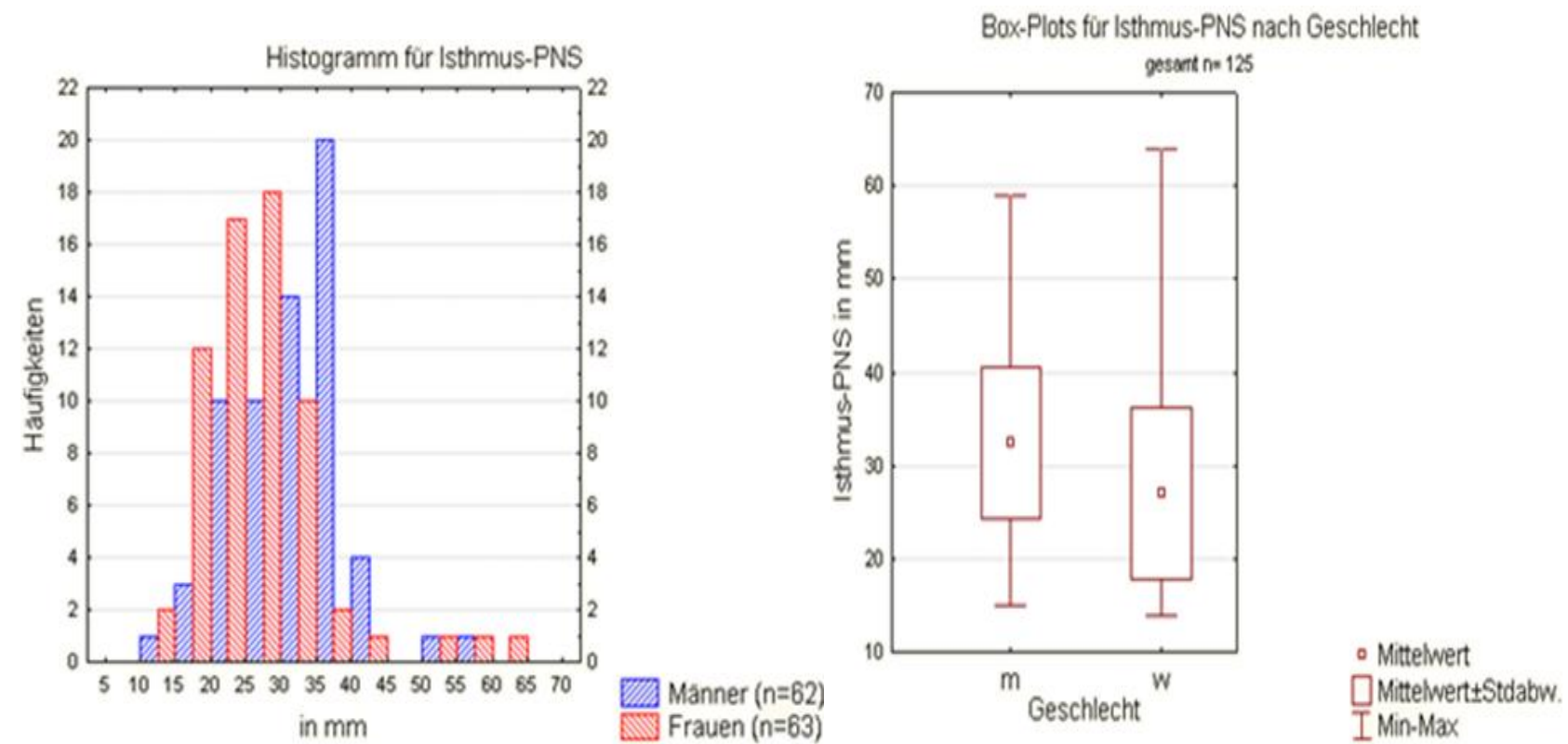

Abb. 21: Box-Plots und Histogramme für die Strecke Isthmus-Nasallinie

Der Mittelwert für den Abstand Isthmus-Nasallinie betrug für die Männer 32,47 mm \pm 8,19 und für die Frauen 26,69 $\mathrm{mm} \pm 9,19$. Der Vergleich beider Kollektive ergab einen $\mathrm{p}$-Wert $<0,001$, somit bestand ein signifikanter Unterschied zwischen beiden Kollektiven, dabei wiesen Männer eine größere Strecke Isthmus-Nasallinie auf als Frauen. 


\subsubsection{Messungen unter Bezug auf axiale Schichten}

Im folgenden werden die Messwerte in den axialen Ebenen dargestellt. Dabei wird von kranial nach kaudal vorgegangen, d.h. es wird mit den Messwerten auf Höhe der Nasallinie (UPW) begonnen, danach folgen die Messwerte auf Höhe Isthmus, auf Höhe des unteren Inzisialpunktes (MPW), auf Höhe des dorsalsten Punktes der Zunge (TPW) und schließlich auf Höhe der Vallecula glossoepiglottica (LPW) (siehe Abb.9 H5-H1). Bei jeder Messebene werden erst die anteriorposterioren Messwerte, dann die transversalen und zum Schluss die Luftwegsflächen an. gegeben.

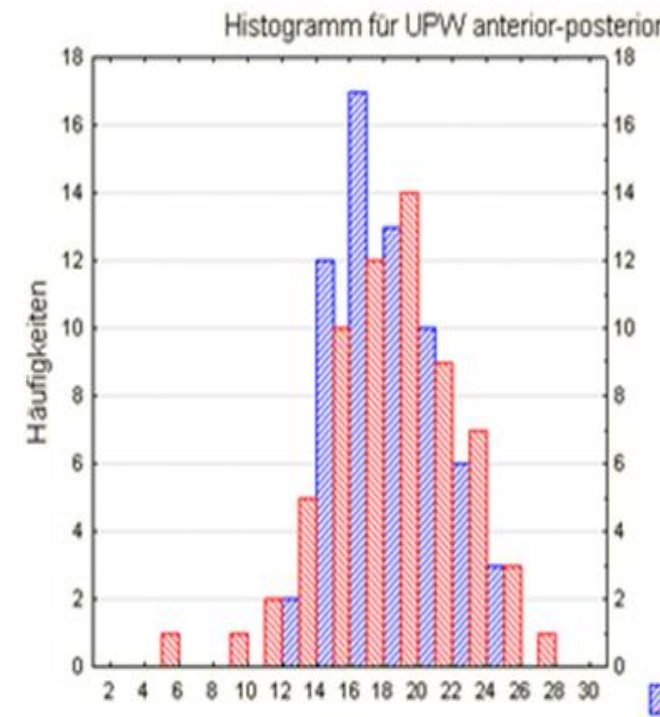

in $\mathrm{mm}$

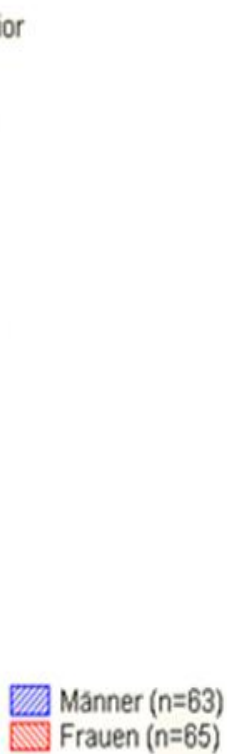

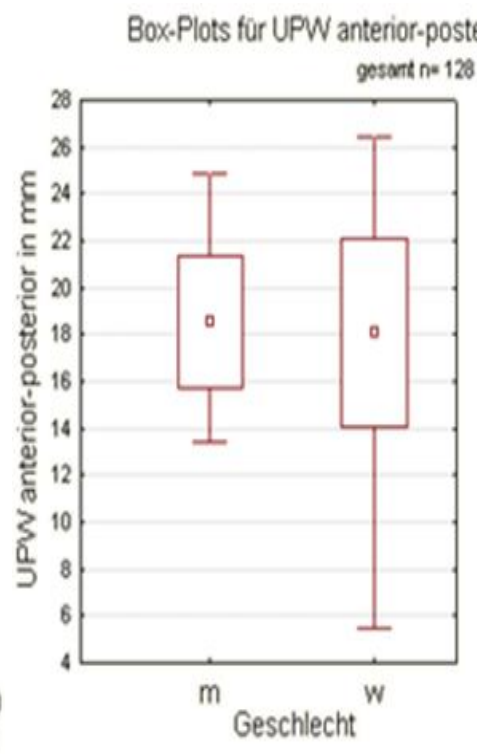

Abb. 22: Box-Plots und Histogramme für die Strecke UPW anterior-posterior

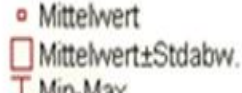
I Min-Max

Der Mittelwert für die Strecke auf Höhe der Nasallinie (UPW) anterior-posterior betrug für die Männer 18,53 mm $\pm 2,84$ und für die Frauen 18,08 $\mathrm{mm} \pm 4,01$. Der Vergleich beider Kollektive ergab einen p-Wert von 0,579, somit bestand kein signifikanter Unterschied zwischen beiden Kollektiven, dabei war die Strecke UPW anterior-posterior bei Männern geringfügig größer als bei Frauen. 

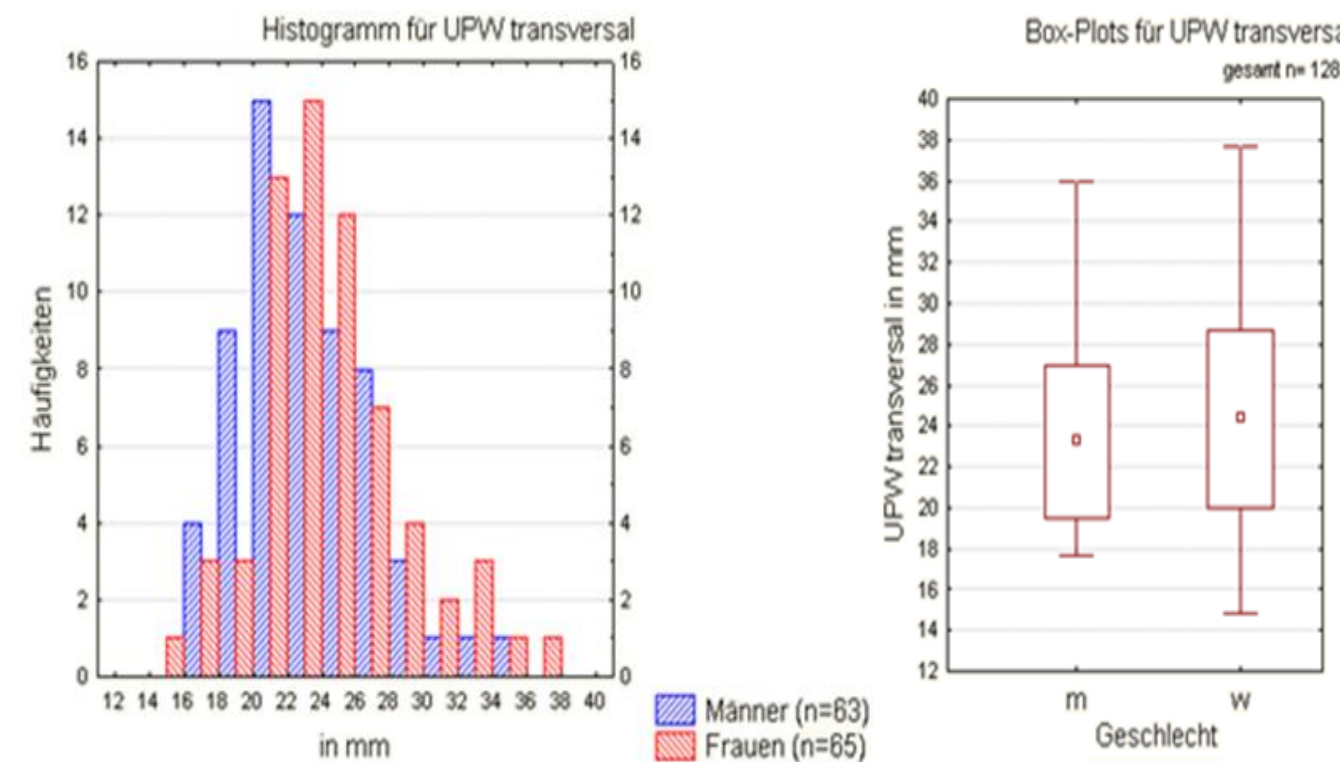

Abb. 23: Box-Plots und Histogramme für die Strecke UPW transversal

Der Mittelwert für die Strecke UPW transversal betrug für die Männer 23,23 mm \pm 3,76 und für die Frauen 24,36 $\mathrm{mm} \pm 4,34$. Der Vergleich beider Kollektive ergab einen p-Wert von 0,093, somit bestand kein signifikanter Unterschied zwischen beiden Kollektiven, dabei war die Strecke UPW transversal bei Frauen größer als bei Männern.
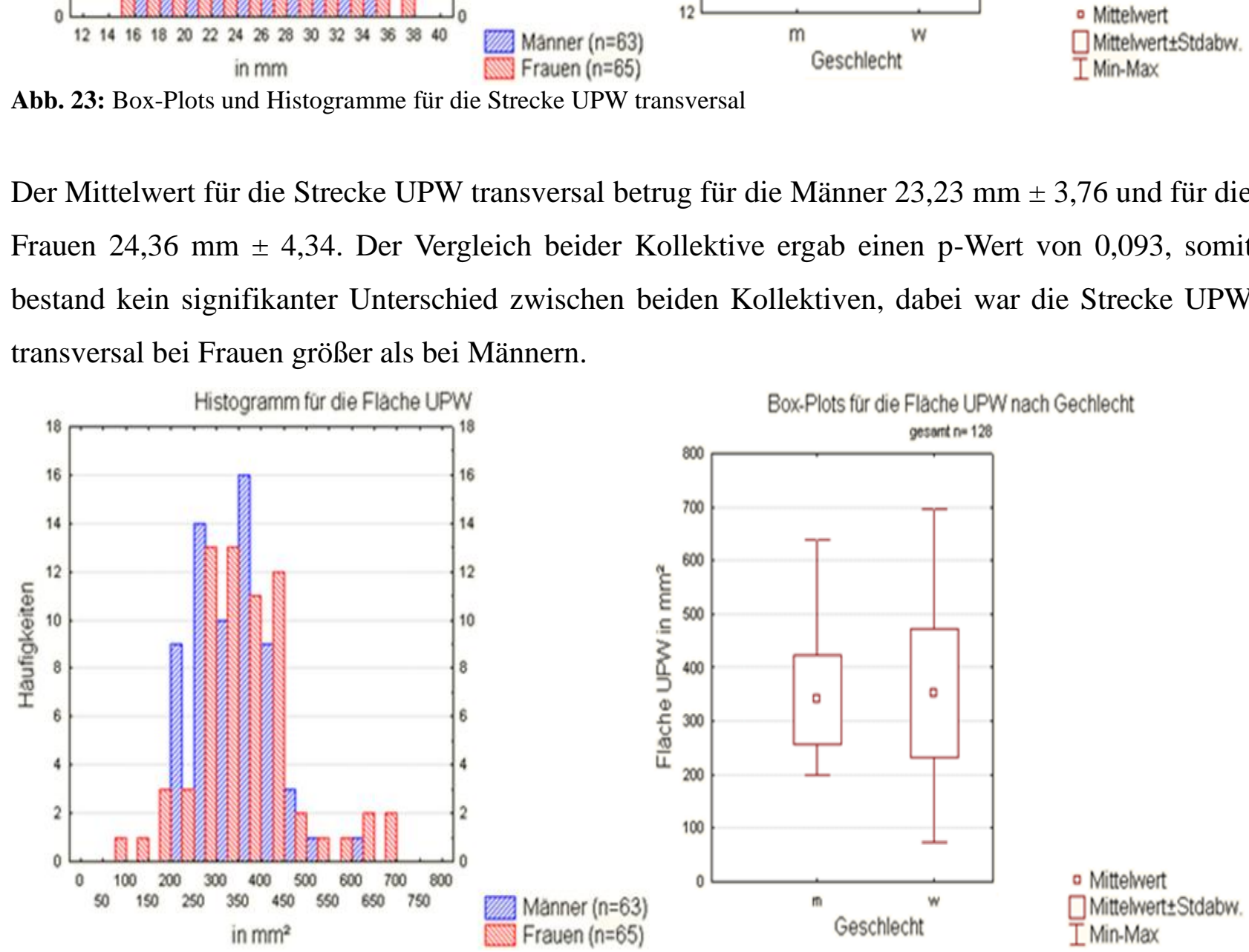

Abb. 24: Box-Plots und Histogramme für die Fläche UPW

Der Mittelwert für die Querschnittsfläche auf Höhe der Nasallinie (Fläche UPW) betrug für die Männer 340,10 $\mathrm{mm}^{2} \pm 84,84$ und für die Frauen 352,20 $\mathrm{mm}^{2} \pm 119,10$. Der p-Wert beider Kollektive lag bei 0,622, somit bestand kein signifikanter Unterschied zwischen beiden Kollektiven. Frauen wiesen eine größere UPW Fläche auf als Männer. 

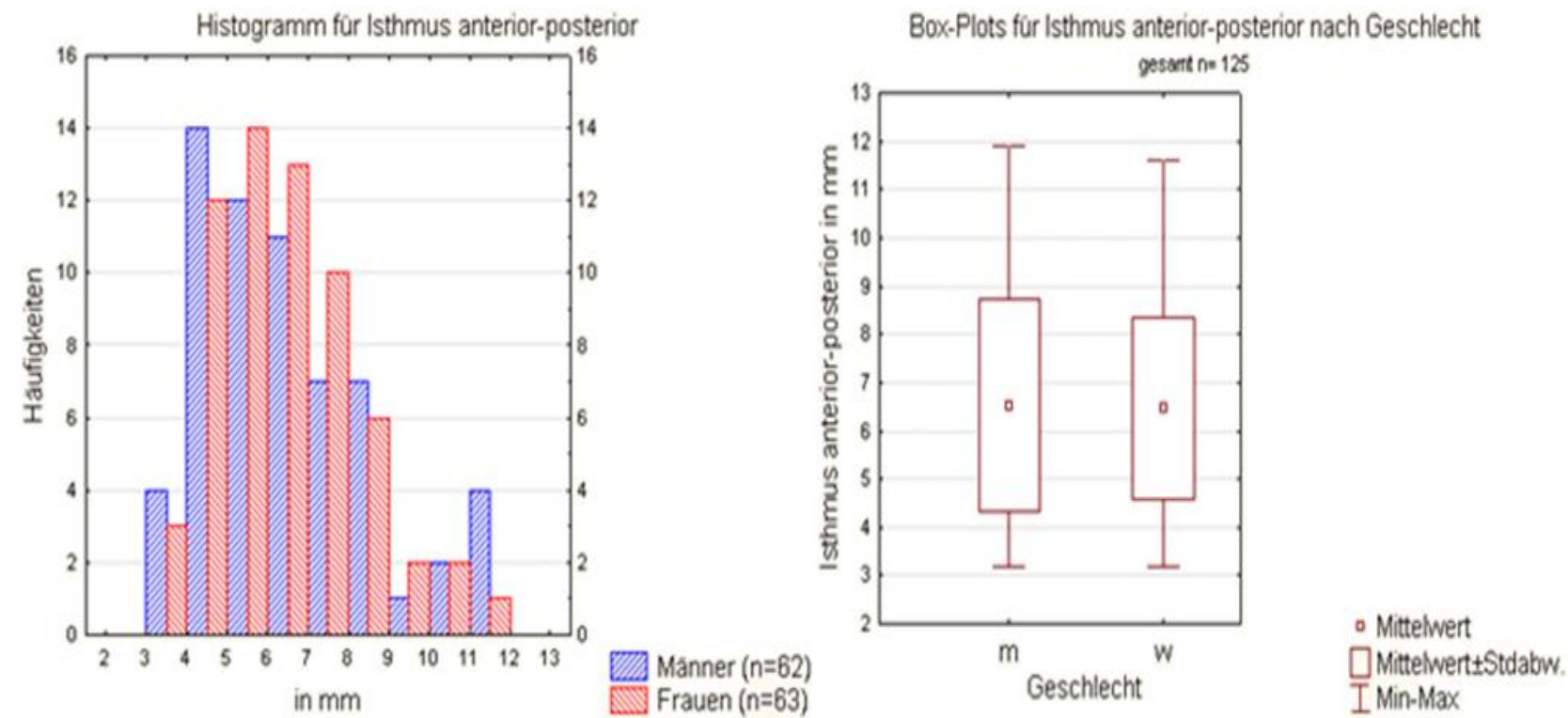

Abb. 25: Box-Plots und Histogramme für die Strecke Isthmus anterior-posterior

Der Mittelwert für die Strecke Isthmus anterior-posterior betrug für Männer 6,52 $\mathrm{mm} \pm 2,19$ und für Frauen $6,46 \mathrm{~mm} \pm 1,87$. Der Vergleich beider Kollektive ergab einen p-Wert von 0,828, somit bestand kein signifikanter Unterschied zwischen beiden Kollektiven. Bei Männern war die Strecke Isthmus anterior-posterior geringfügig größer als bei Frauen.
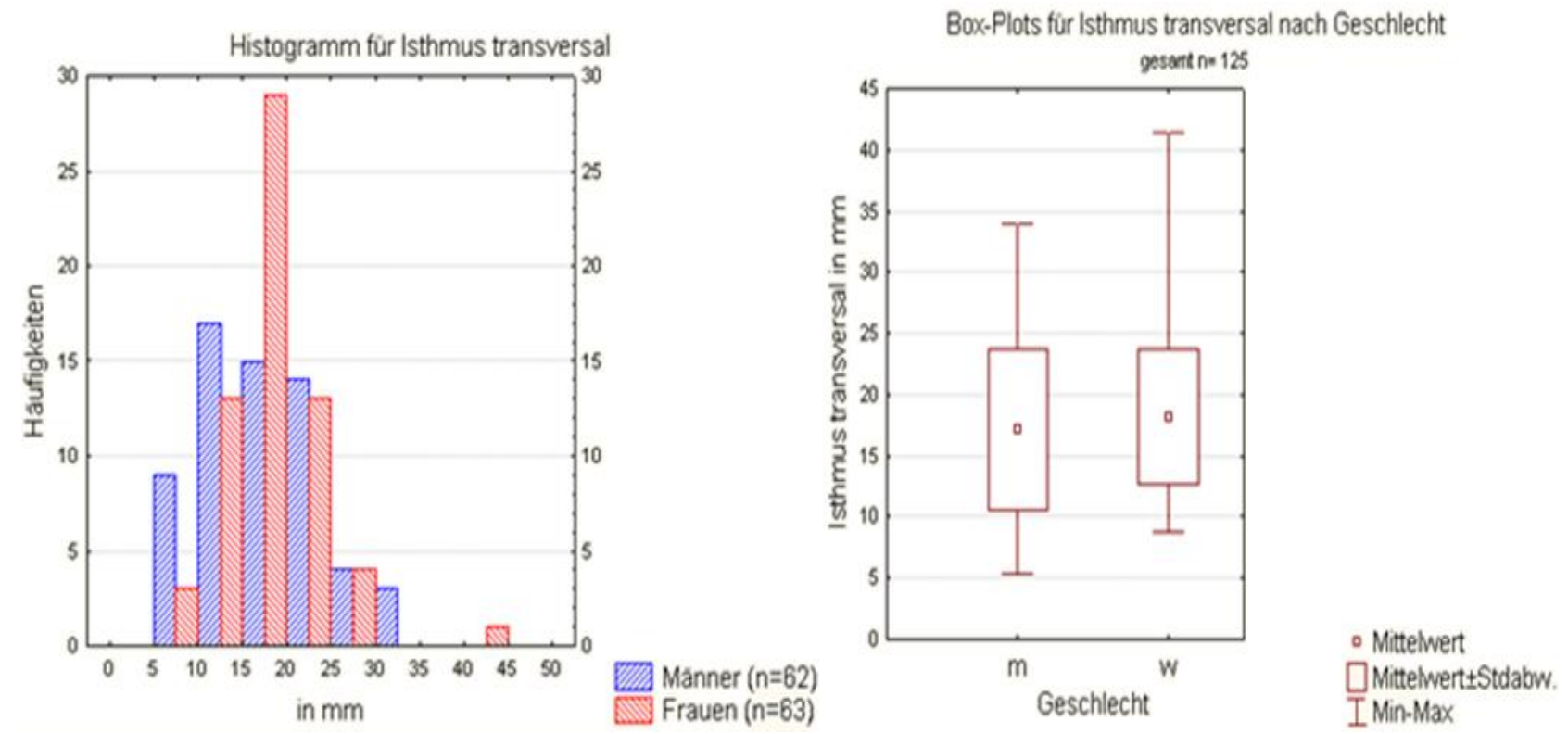

Abb. 26: Box-Plots und Histogramme für die Strecke Isthmus transversal

Der Mittelwert für die Strecke Isthmus transversal betrug für Männer 17,14 mm \pm 6,54 und für Frauen $18,19 \mathrm{~mm} \pm 5,48$. Der Vergleich beider Kollektive ergab einen p-Wert von 0,256, somit bestand kein signifikanter Unterschied zwischen beiden Kollektiven. Bei Frauen war die Strecke Isthmus transversal größer als bei Männern. 

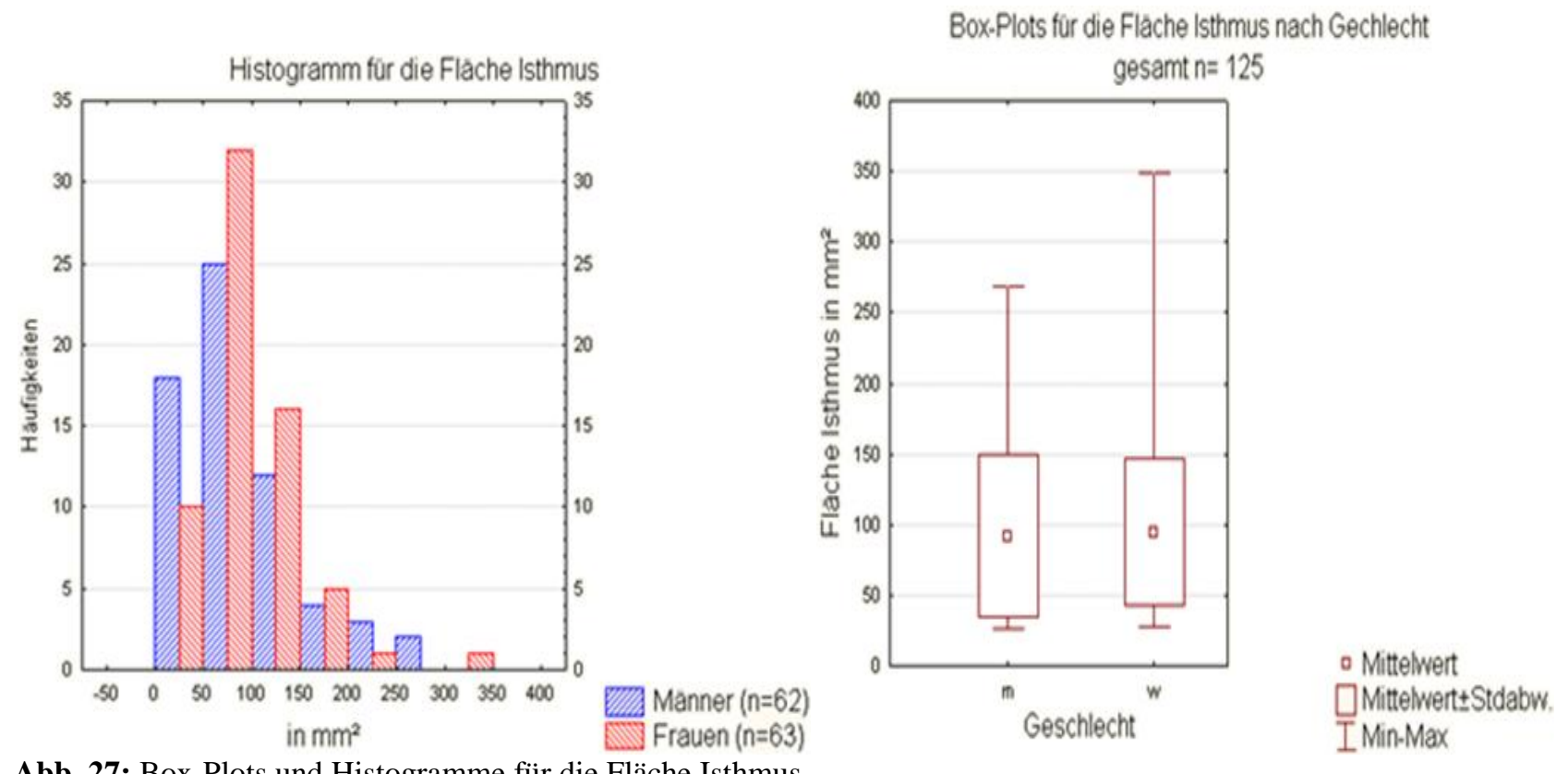

Abb. 27: Box-Plots und Histogramme für die Fläche Isthmus

Der Mittelwert für die Fläche Isthmus betrug für Männer 91,82 mm² \pm 57,74 und für Frauen 94,89 $\mathrm{mm}^{2} \pm 51,98$. Der p-Wert beider Kollektive lag bei 0,249, somit bestand kein signifikanter Unterschied zwischen den Kollektiven. Bei Frauen war die Fläche Isthmus größer als bei Männern.

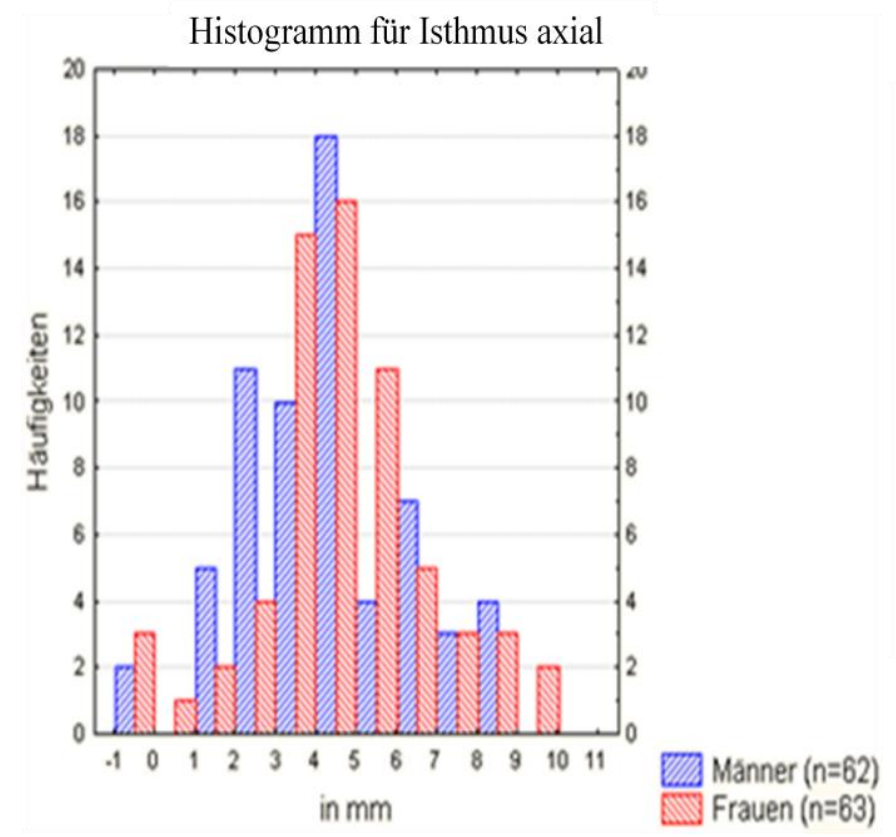

Box-Plots für Isthmus axial nach Geschlecht gesamt $n=125$

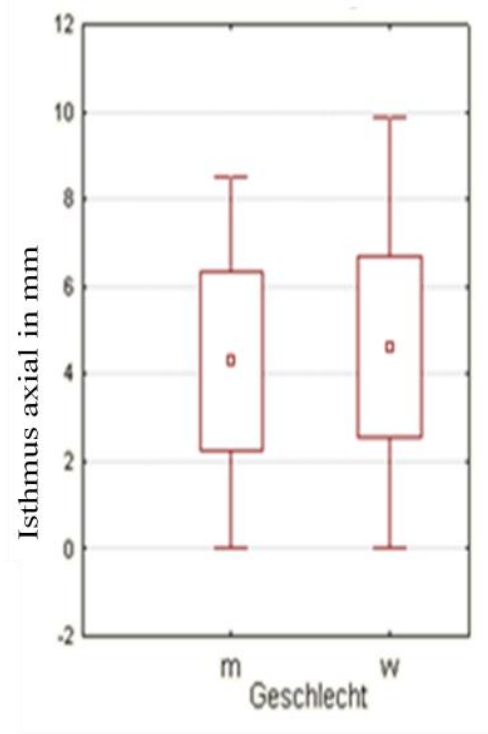

Abb. 28: Box-Plots und Histogramme für die Strecke Isthmus axial

Der Mittelwert für die Strecke Isthmus axial in anterior-posteriorer Richtung betrug für Männer 4,28 $\mathrm{mm} \pm 2,04$ und für Frauen 4,62 mm $\pm 2,05$. Der Vergleich beider Kollektive ergab einen $\mathrm{p}$ Wert von 0,264, somit bestand kein signifikanter Unterschied zwischen beiden Kollektiven. Bei Frauen war die Strecke Isthmus axial größer als bei Männern. 

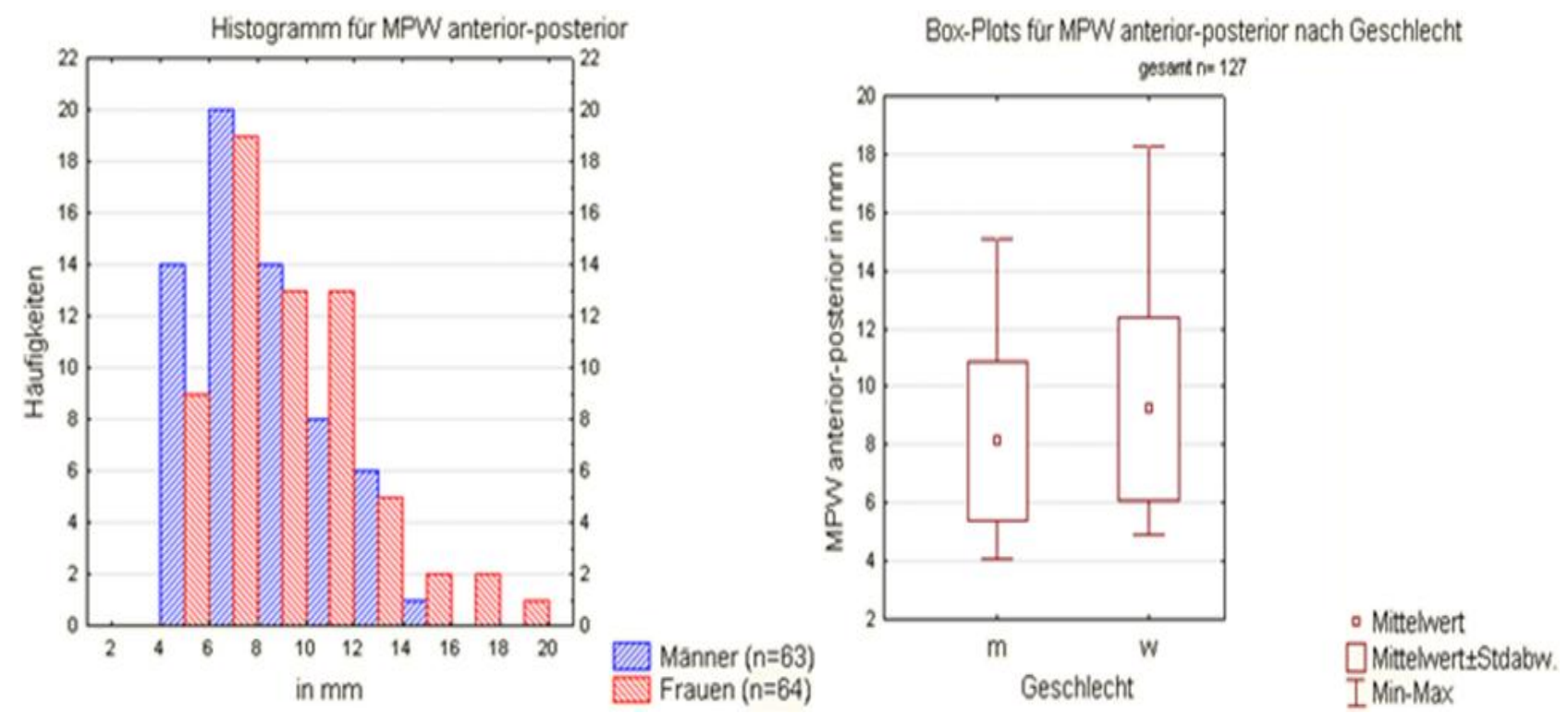

Abb. 29: Box-Plots und Histogramme für die Strecke MPW anterior-posterior

Der Mittelwert für die Strecke auf Höhe des untern Inzisialpunktes (MPW) anterior-posterior betrug für Männer 8,14 mm \pm 2,75 und für Frauen 9,24 mm $\pm 3,15$. Der Vergleich beider Kollektive ergab einen p-Wert von 0,045, somit bestand ein signifikanter Unterschied zwischen beiden Kollektiven. Frauen wiesen eine größere Strecke MPW anterior-posterior auf als Männer.
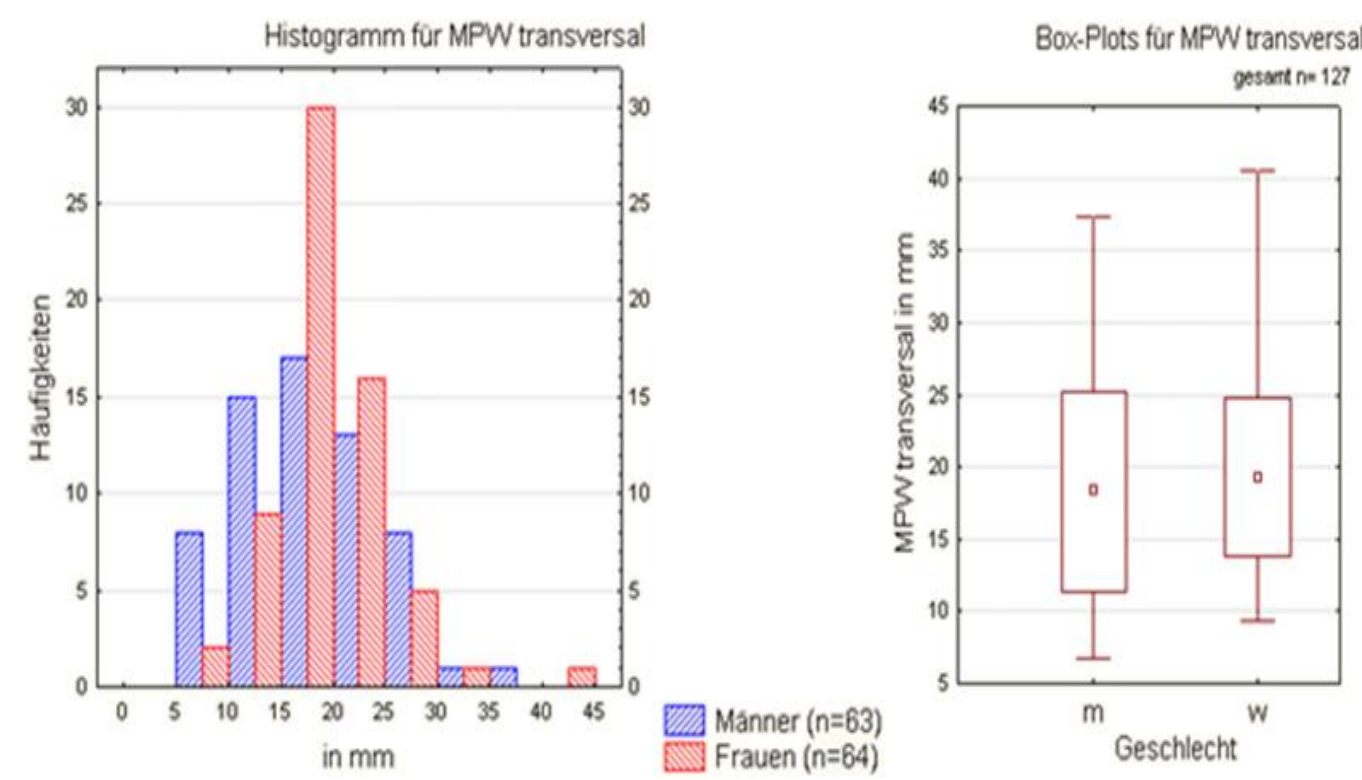

Abb. 30: Box-Plots und Histogramme für die Strecke MPW transversal

Der Mittelwert für die Strecke MPW transversal betrug für Männer 18,30 mm \pm 6,88 und für Frauen 19,27 mm \pm 5,50. Der Vergleich beider Kollektive ergab einen p-Wert von 0,325, somit bestand kein signifikanter Unterschied zwischen beiden Kollektiven. Frauen wiesen eine größere Strecke MPW transversal auf als Männer. 


\section{Ergebnisse}
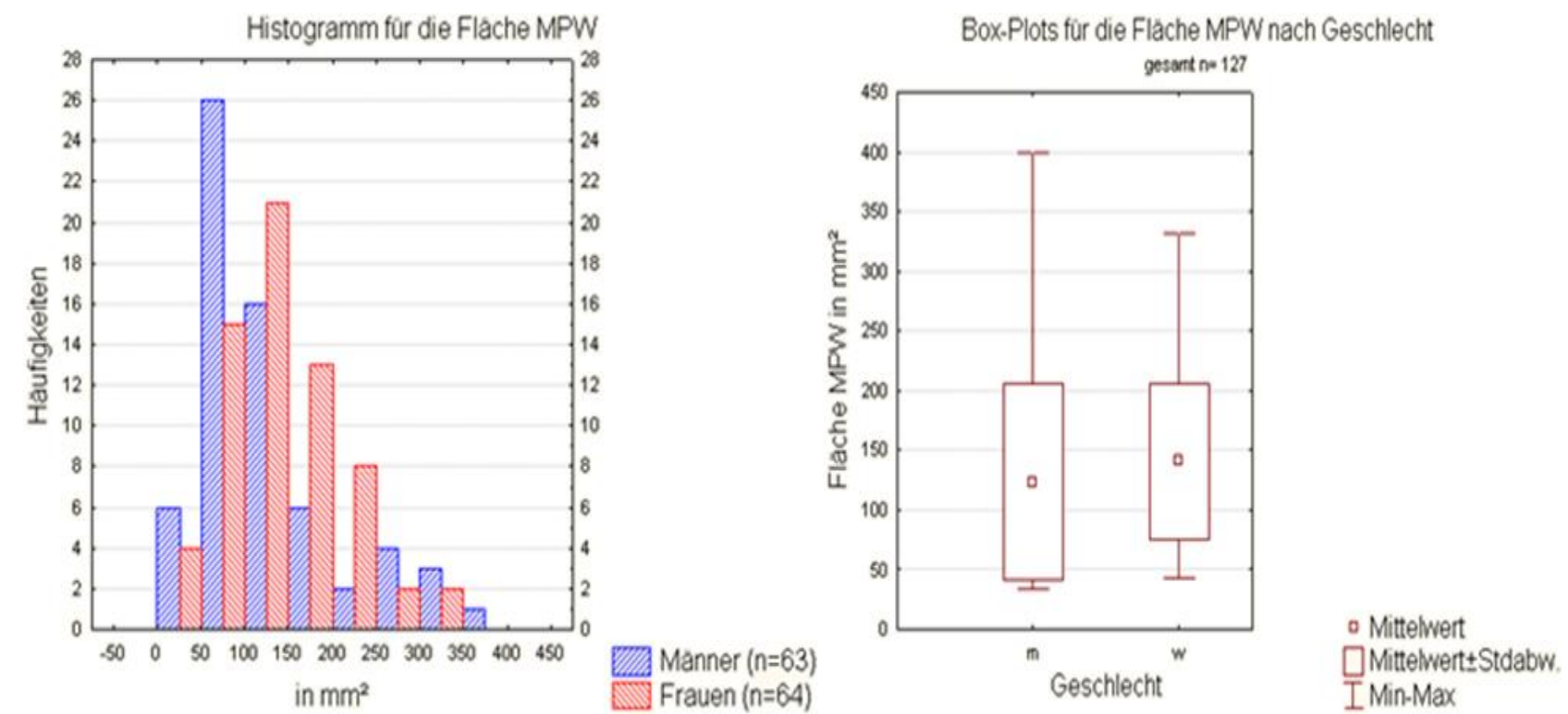

Abb. 31: Box-Plots und Histogramme für die Fläche MPW

Mânner ( $n=63)$ Frauen $(n=64)$

Der Mittelwert für die Querschnittsfläche auf Höhe des unteren Inzisialpunkter (Fläche MPW) betrug für Männer 123,37 $\mathrm{mm}^{2} \pm 82,52$ und für Frauen 140,97 $\mathrm{mm}^{2} \pm 64,98$. Der p-Wert beider Kollektive lag bei 0,014, somit bestand ein signifikanter Unterschied zwischen beiden Kollektiven, dabei war die Fläche MPW bei Frauen größer als bei Männern.
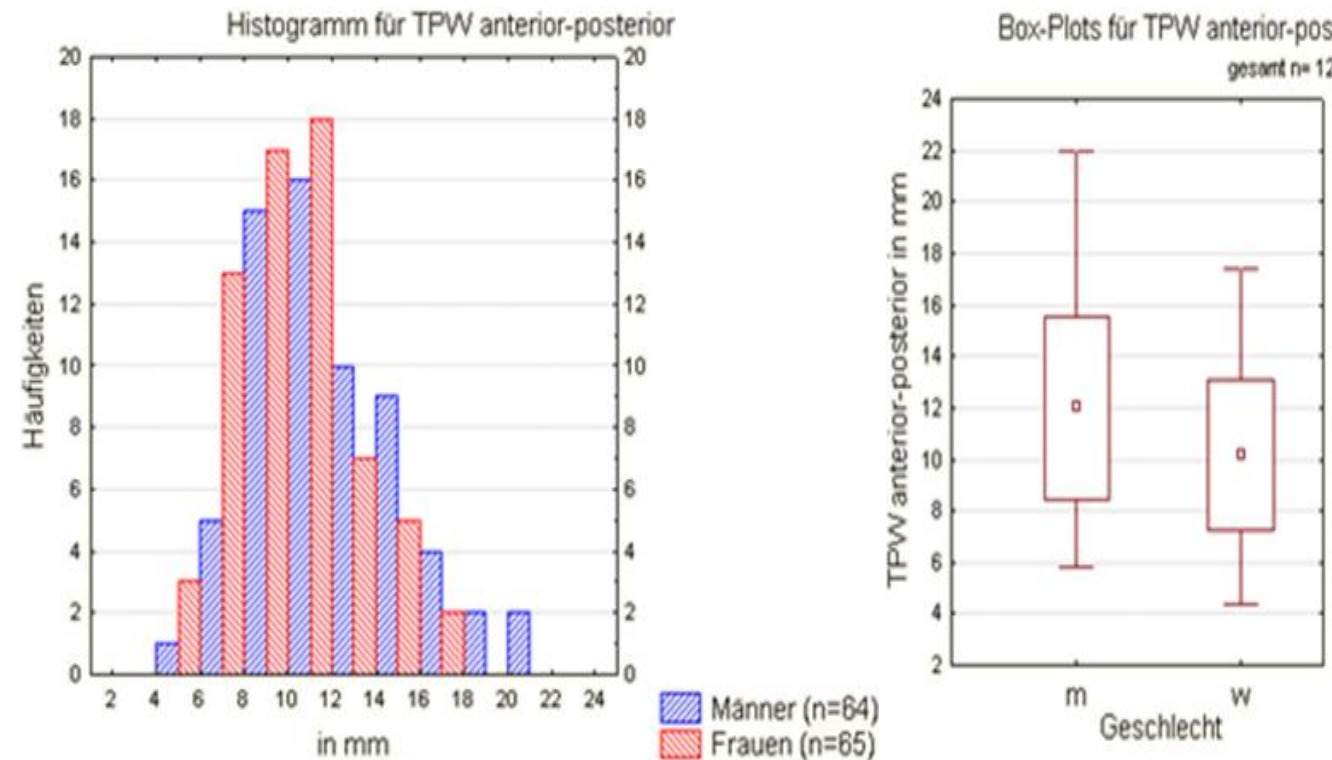

Abb. 32: Box-Plots und Histogramme für die Strecke TPW anterior-posterior

Der Mittelwert für die Strecke auf Höhe des dorsalsten Punktes der Zungen (TPW) anteriorposterior betrug für Männer 11,99 $\mathrm{mm} \pm 3,56$ und für Frauen 10,14 mm $\pm 2,91$. Der Vergleich beider Kollektive ergab einen p-Wert von 0,004, somit bestand ein signifikanter Unterschied zwischen beiden Kollektiven. Bei Männern war die Strecke TPW anterior-posterior größer als bei Frauen. 

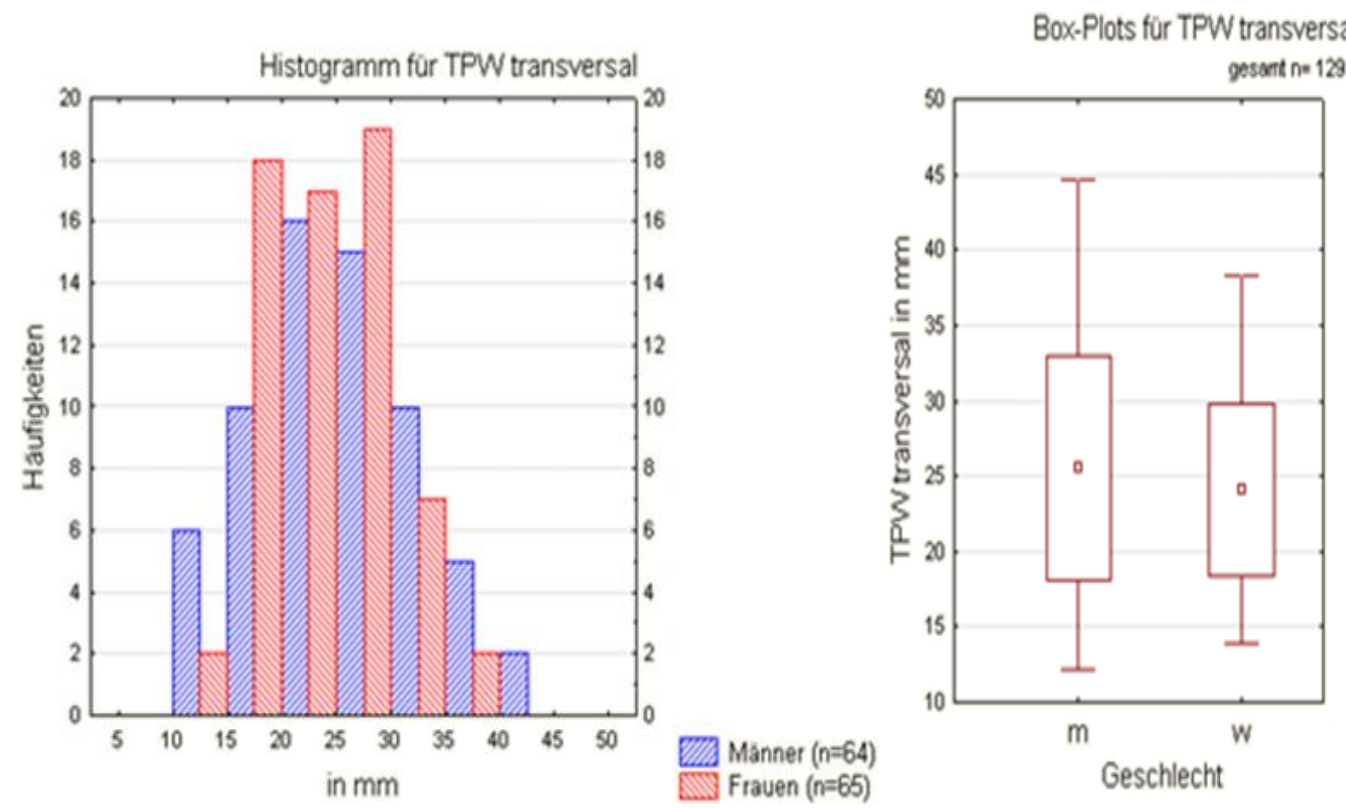

Abb. 33: Box-Plots und Histogramme für die Strecke TPW transversal

Der Mittelwert für die Strecke TPW transversal betrug für Männer 25,55 mm \pm 7,38 und für Frauen $24,15 \mathrm{~mm} \pm 5,70$. Der Vergleich beider Kollektive ergab einen p-Wert von 0,343, somit bestand kein signifikanter Unterschied zwischen beiden Kollektiven, dabei war die Strecke TPW transversal bei Männern größer als bei Frauen.
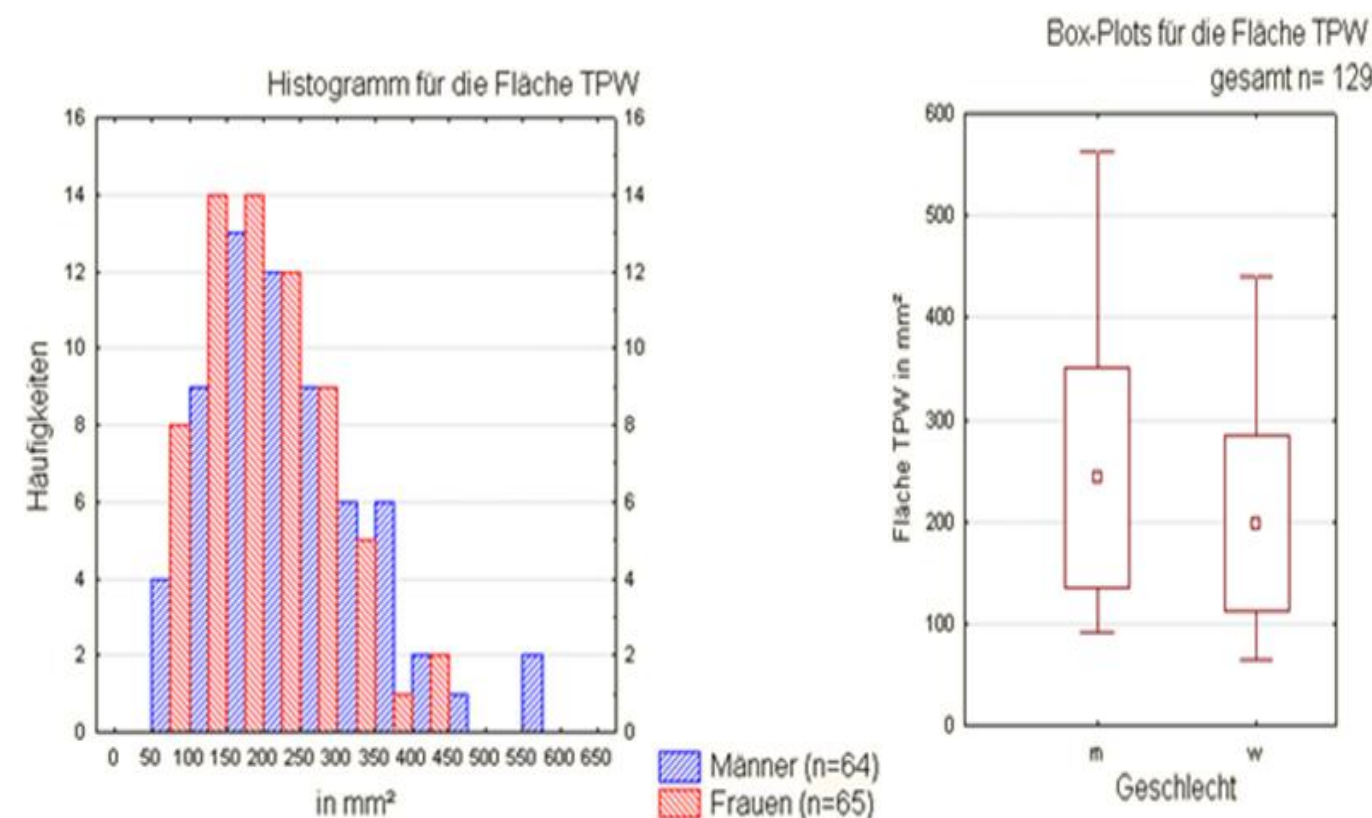

Abb. 34: Box-Plots und Histogramme für die Fläche TPW

Der Mittelwert für die Querschnittsfläche auf Höhe des dorsalsten Punktes der Zunge (Fläche TPW) betrug für Männer 243,63 mm² $\pm 108,31$ und für Frauen 197,89 mm² $\pm 86,54$. Der p-Wert beider Kollektive lag bei 0,017, somit bestand ein signifikanter Unterschied zwischen beiden Kollektiven. Die Fläche TPW war bei Männern größer als bei Frauen. 

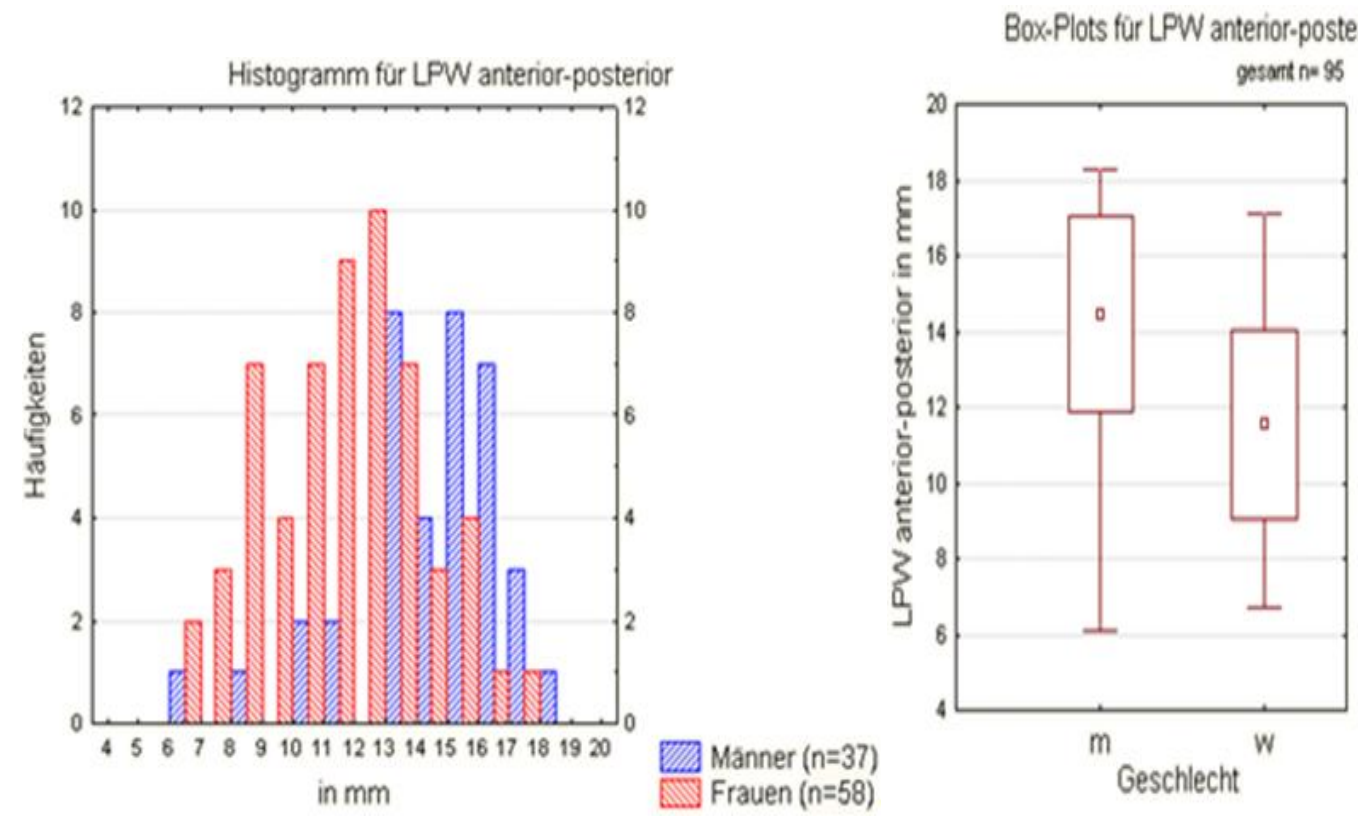

Abb. 35: Box-Plots und Histogramme für die Strecke LPW anterior-posterior

Der Mittelwert für die Strecke auf Höhe der Vallecula glossoepiglottica (LPW) anterior-posterior betrug für Männer 14,46 mm $\pm 2,61$ und für Frauen 11,55 mm $\pm 2,50$. Der Vergleich beider Kollektive ergab einen $\mathrm{p}$-Wert $<0,001$, somit bestand ein signifikanter Unterschied zwischen beiden Kollektiven, dabei war die Strecke LPW anterior-posterior bei Männern größer als bei Frauen.
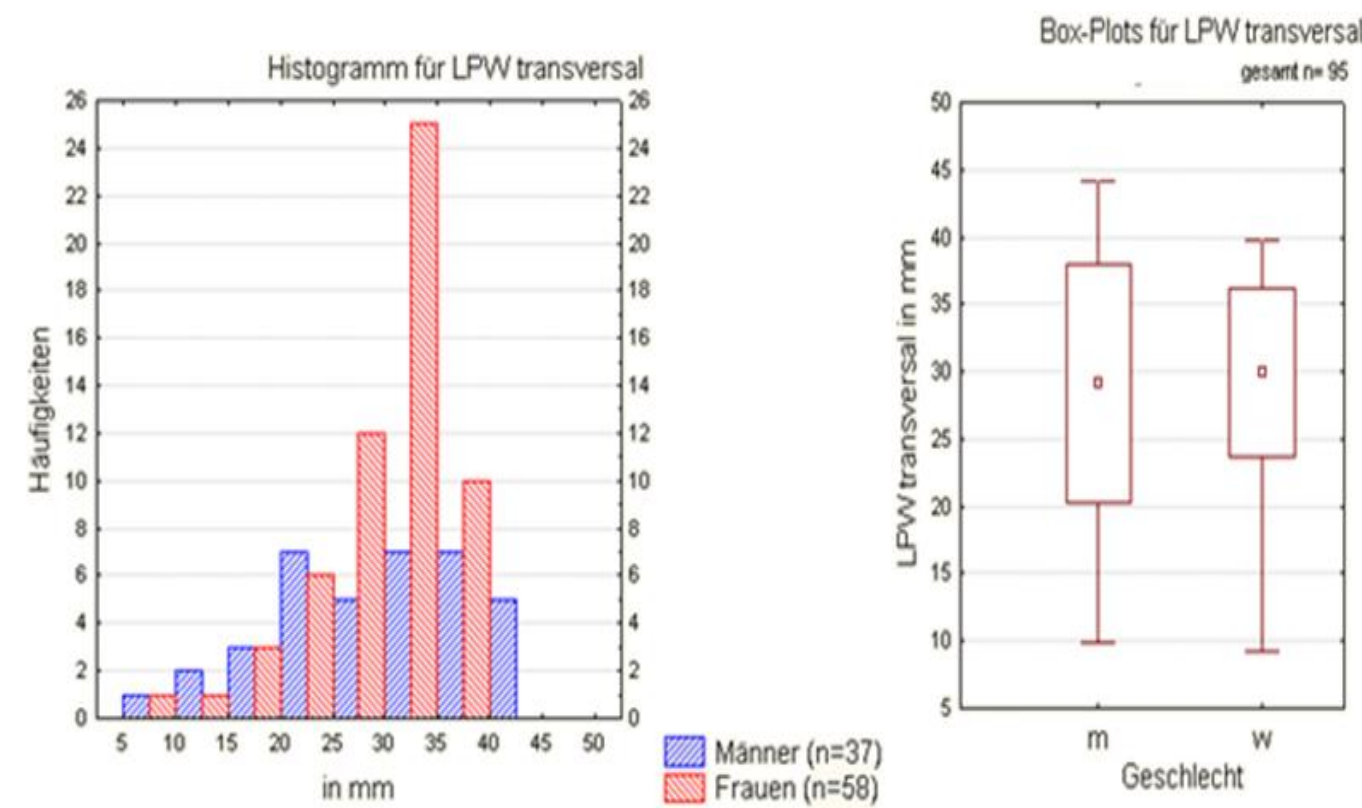

Abb. 36: Box-Plots und Histogramme für die Strecke LPW transversal

Der Mittelwert für die Strecke LPW transversal betrug für Männer 29,20 mm \pm 8,85 und für Frauen $29,98 \mathrm{~mm} \pm 6,25$. Der Vergleich beider Kollektive ergab einen p-Wert von 0,939, somit bestand kein signifikanter Unterschied zwischen beiden Kollektiven, dabei war die Strecke LPW transversal bei Frauen geringfügig größer als bei Männern. 

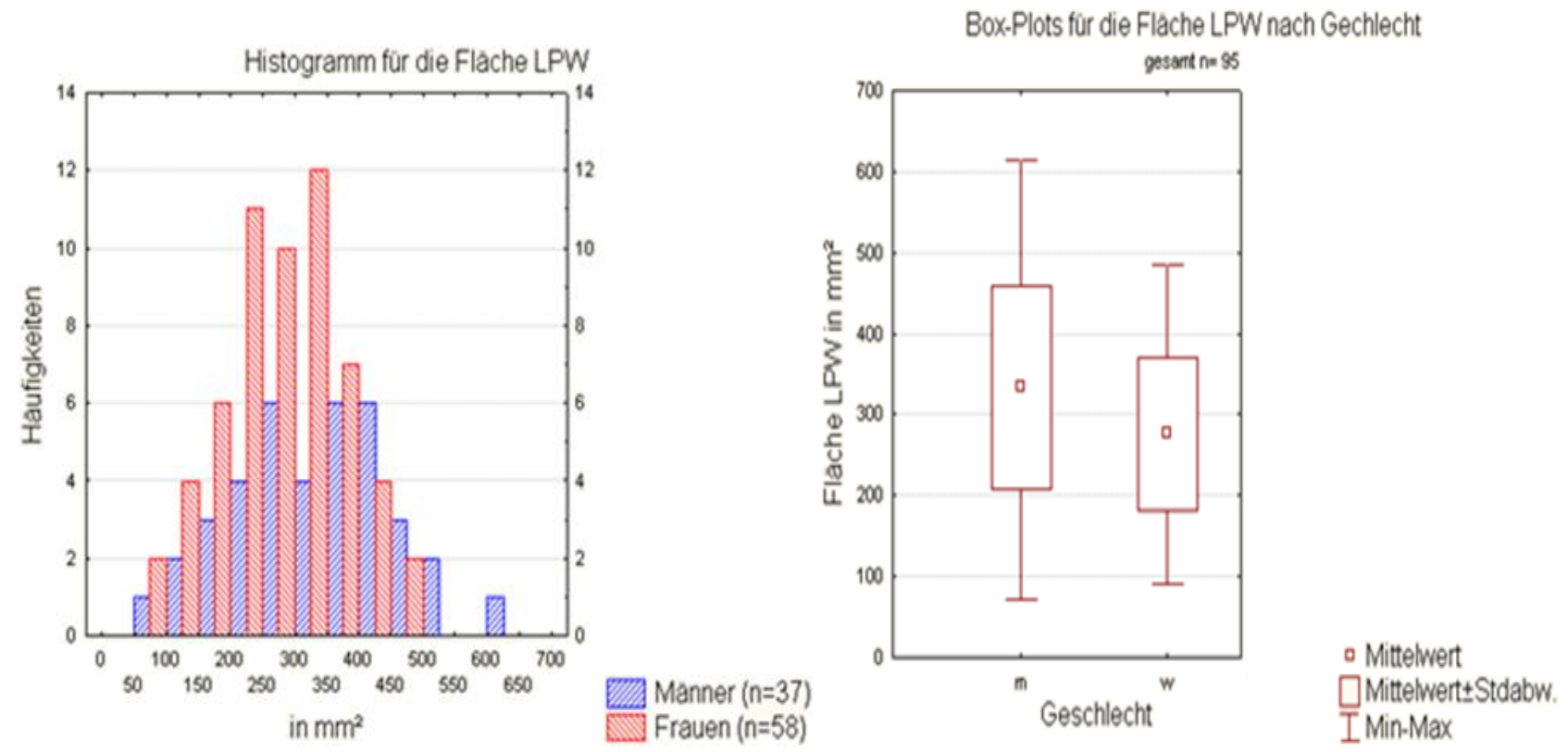

Abb. 37: Box-Plots und Histogramme für die Fläche LPW

Der Mittelwert für die Querschnittsfläche auf Höhe der Vallecula glossoepiglottica (Fläche LPW) betrug für Männer 333,94 mm² $\pm 125,91$ und für Frauen 276,06 mm² $\pm 94,56$. Der p-Wert beider Kollektive lag bei 0,018, somit bestand ein signifikanter Unterschied zwischen beiden Kollektiven, dabei war die Fläche LPW bei Männern größer als bei Frauen.
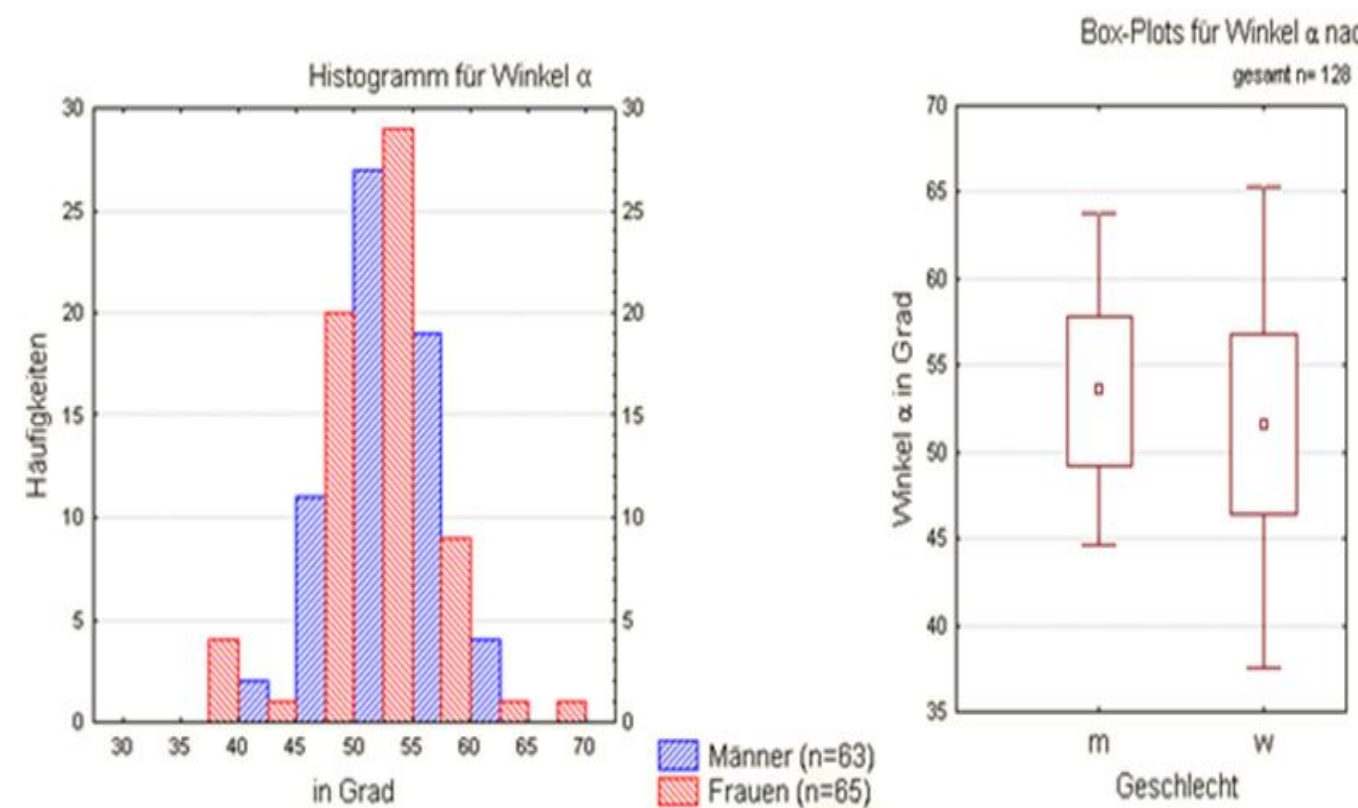

Abb. 38: Box-Plots und Histogramme für den Winkel $\alpha$

Mănner $(n=63)$

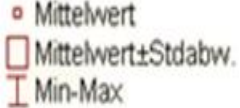

Der Mittelwert für den Winkel $\alpha$ betrug für Männer 53,52 $\pm 4,28$ und für Frauen $51,60^{\circ} \pm 5,21$. Der Vergleich beider Kollektive ergab einen p-Wert von 0,032, somit bestand ein signifikanter Unterschied zwischen beiden Kollektiven. Männer wiesen einen größeren Winkel $\alpha$ auf als Frauen. 

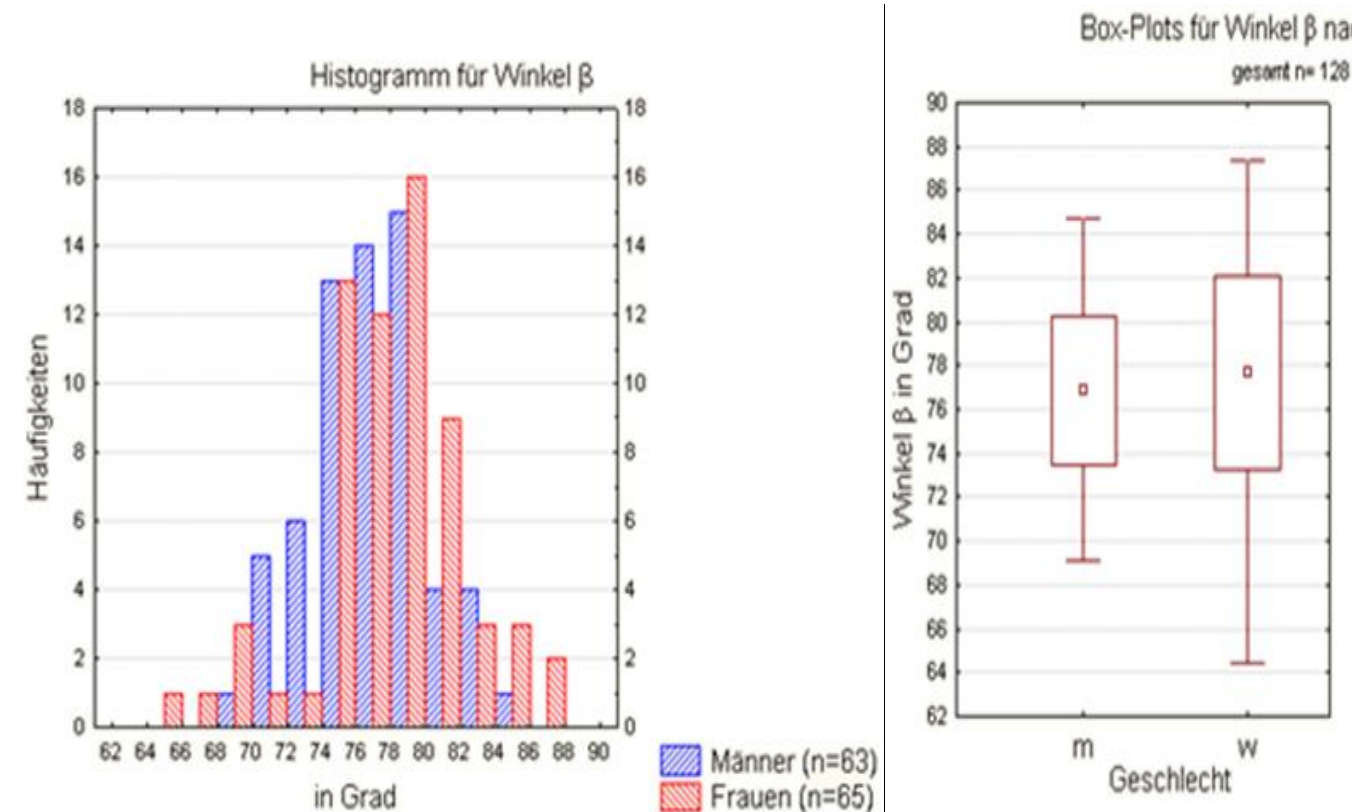

Abb. 39: Box-Plots und Histogramme für den Winkel $\beta$

Der Mittelwert für den Winkel $\beta$ betrug für Männer $76,87^{\circ} \pm 3,40$ und für Frauen $77,71^{\circ} \pm 4,40$.

Der Vergleich beider Kollektive ergab einen p-Wert von 0,097, somit bestand kein signifikanter Unterschied zwischen beiden Kollektiven. Frauen wiesen einen geringfügig größeren Winkel $\beta$ auf als Männer.
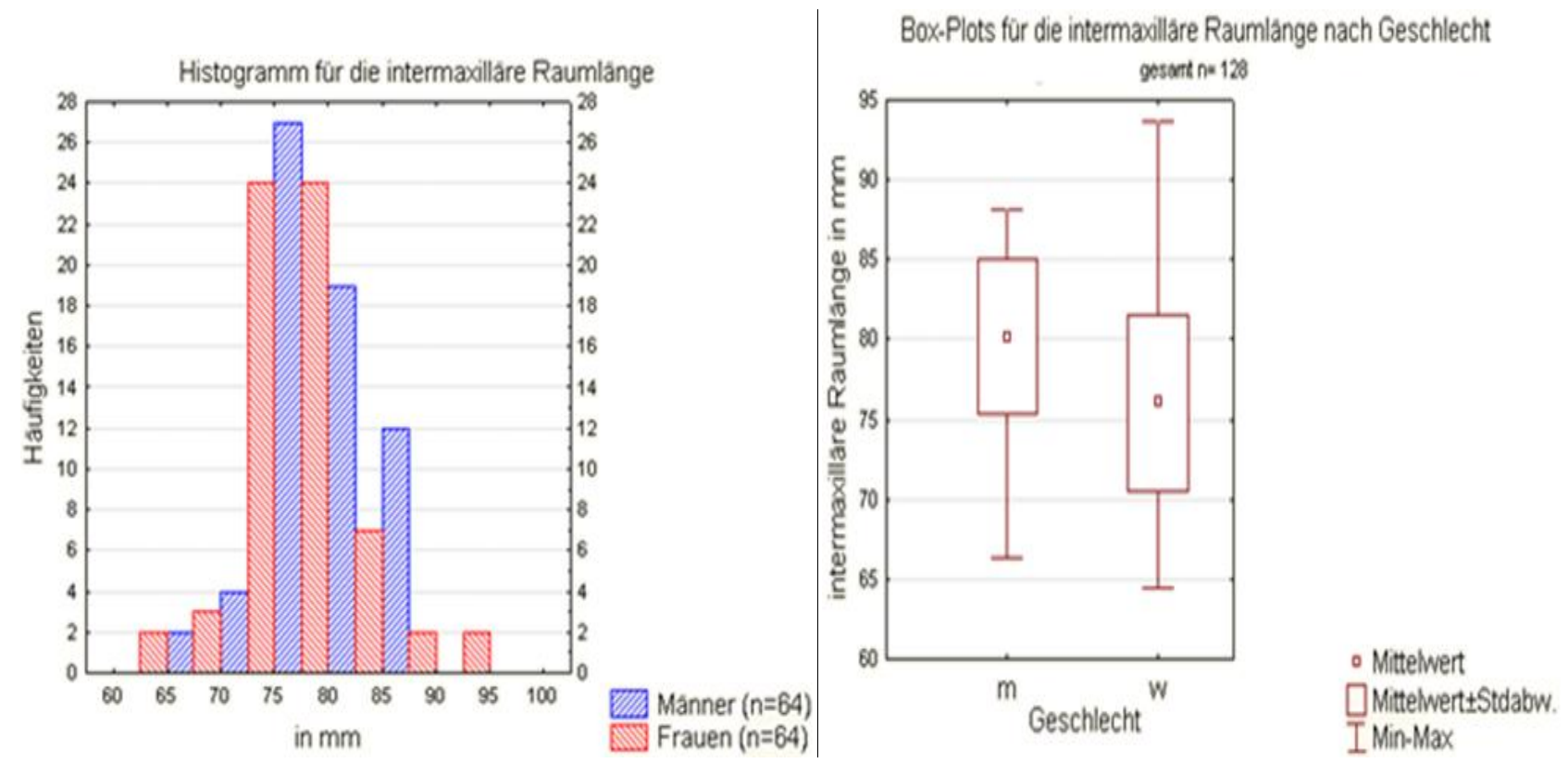

Abb. 40: Box-Plots und Histogramme für die intermaxilläre Raumlänge

Der Mittelwert für die intermaxilläre Raumlänge betrug für die Männer 80,14 mm \pm 4,85 und für die Frauen 76,08 $\mathrm{mm} \pm 5,50$. Der Vergleich beider Kollektive ergab einen $\mathrm{p}$-Wert $<0,001$, somit bestand ein signifikanter Unterschied zwischen beiden Kollektiven. Die intermaxilläre Raumlänge war bei Männern größer als bei Frauen. 


\subsubsection{Koronale Messungen}

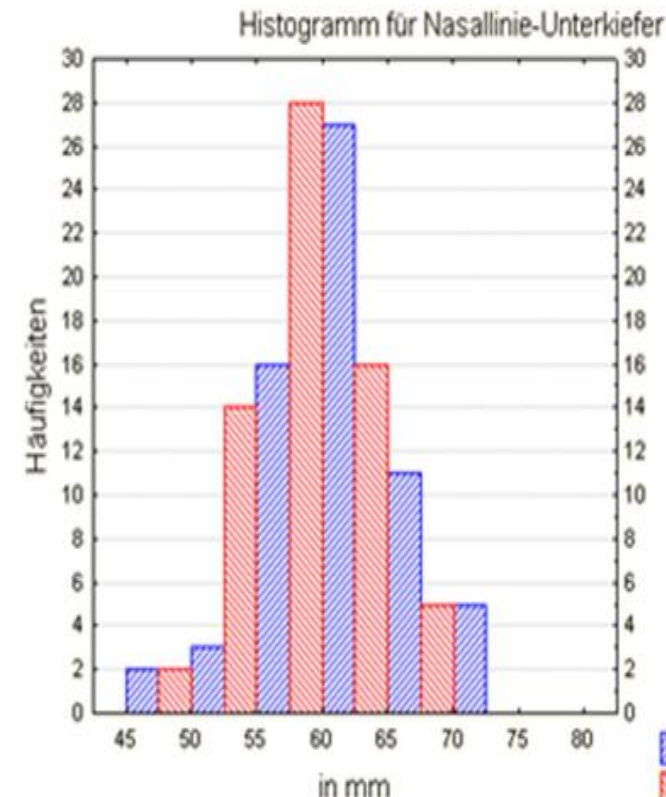

Mănner $(n=64)$

Frauen $(n=65)$

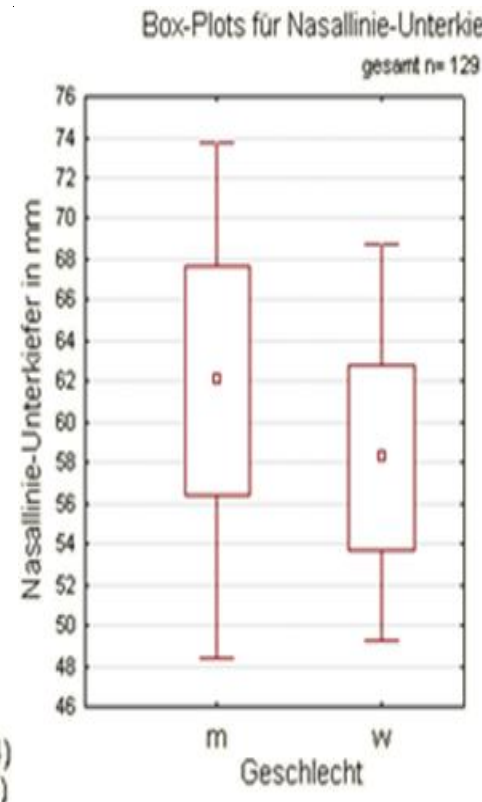

Abb. 41: Box-Plots und Histogramme für die Strecke Nasallinie-Unterkiefer

- Mittelwert $\square$ Mittelwert \pm Stdabw. IMin-Max

Der Mittelwert für die Strecke Nasallinie-Unterkiefer betrug für die Männer 62,04 mm \pm 5,65 und für die Frauen 58,24 mm \pm 4,60. Der Vergleich beider Kollektive ergab einen p-Wert <0,001, somit bestand ein signifikanter Unterschied zwischen beiden Kollektiven. Die Strecke NasallinieUnterkiefer war bei den Männern größer als bei den Frauen.
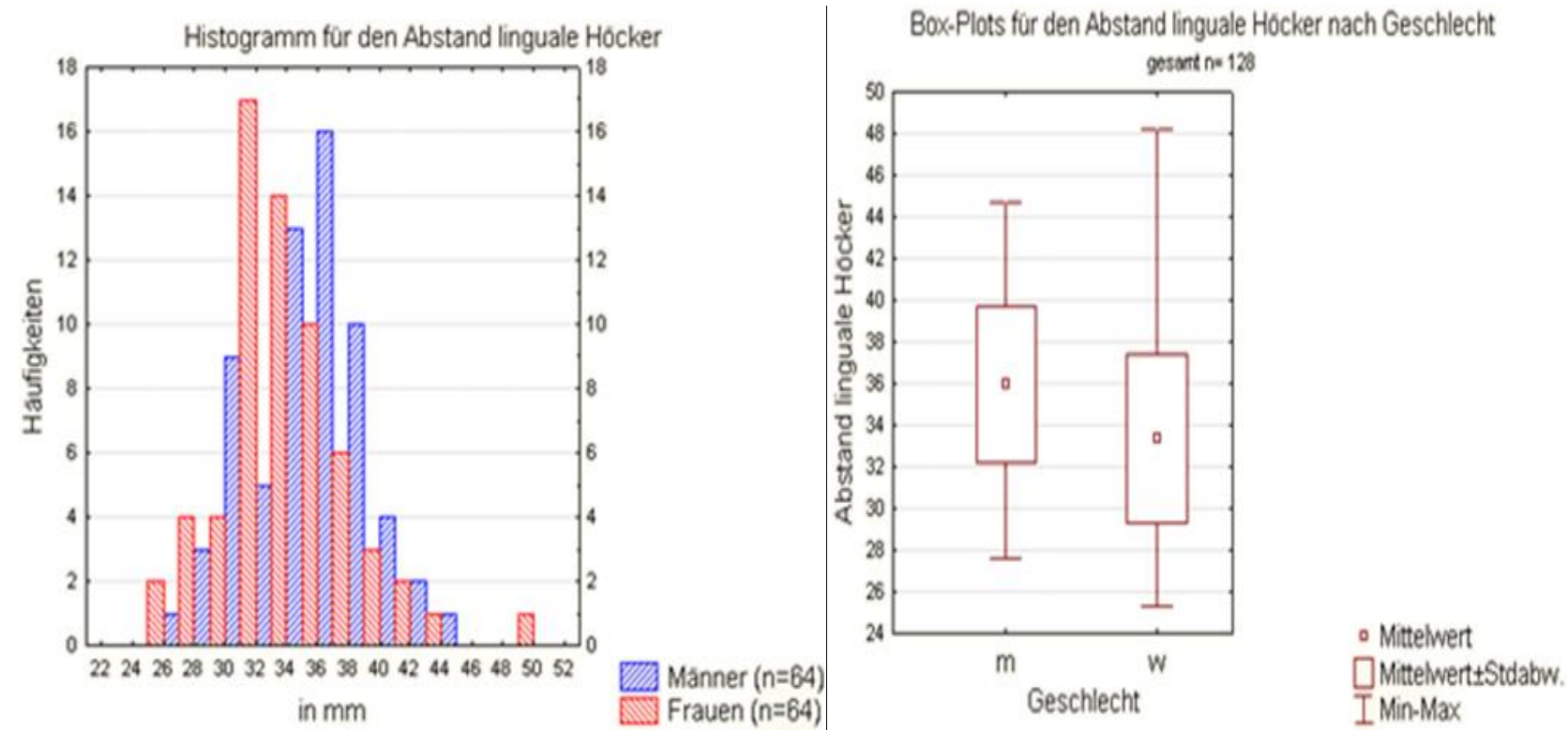

Abb. 42: Box-Plots und Histogramme für den Abstand der lingualen Höcker

Der Mittelwert für die Strecke zwischen den lingualen Höckern im Unterkiefer betrug für die Männer 35,96 mm \pm 3,72 und für die Frauen 33,35 $\mathrm{mm} \pm$ 4,04. Der Vergleich beider Kollektive ergab einen $\mathrm{p}$-Wert <0,001, somit bestand ein signifikanter Unterschied zwischen beiden Kollektiven. Dabei war die Strecke bei Männern größer als bei Frauen. 


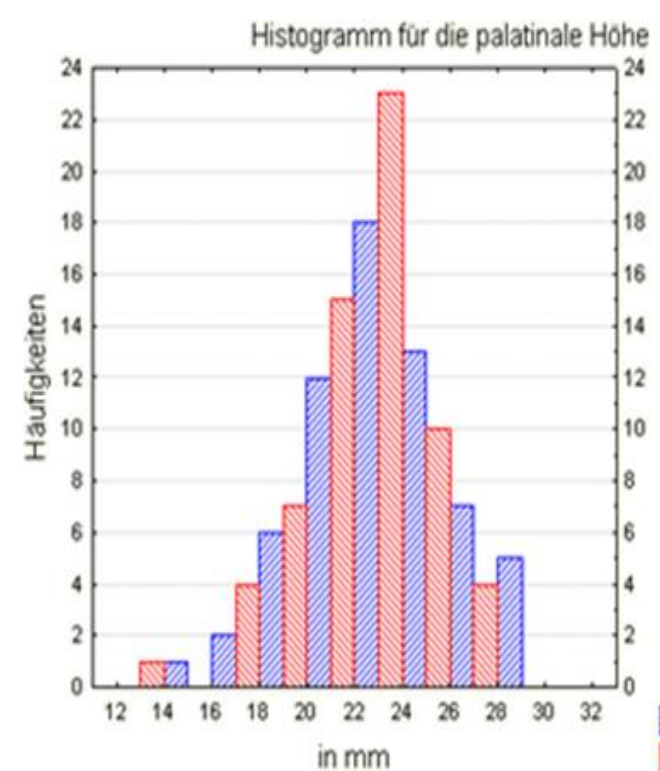

Abb. 43: Box-Plots und Histogramme für die palatinale Höhe

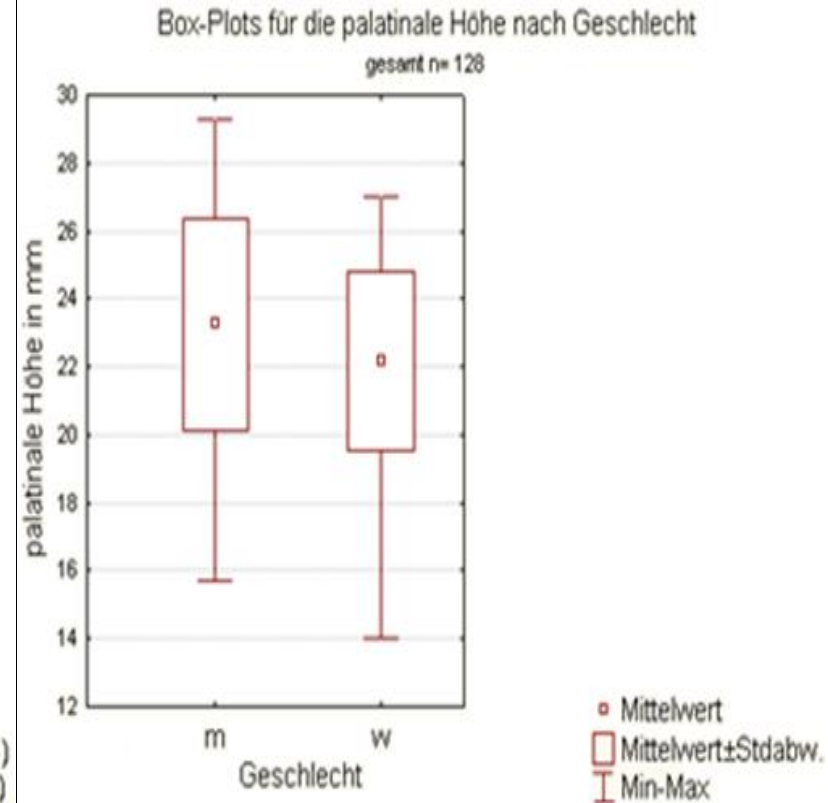

Der Mittelwert für die palatinale Höhe betrug für die Männer 23,26 mm \pm 3,12 und für die Frauen $22,17 \mathrm{~mm} \pm 2,63$. Der Vergleich beider Kollektive ergab einen $\mathrm{p}$-Wert von 0,088, somit bestand kein signifikanter Unterschied zwischen beiden Kollektiven. Bei den Männern ist die palatinale Höhe größer als bei den Frauen. 


\subsection{Korrelation der Messwerte bei Männern}

\subsubsection{Korrelation des kollapsfähigen Segmentes mit ausgewählten Parametern}

Für die Überprüfung einer Korrelation zwischen dem kollapsfähigen Segment (Strecke PNSEpiglottis) und der Weichgaumenlänge, der Strecke Hyoid zu Nasallinie und der subpalatinale Luftraum wurden die Korrelationsmatrizen nach Pearson angewandt.

Tabelle. 4: Korrelation der Strecke PNS-Epiglottis mit ausgewählten Parametern für Männer. Es wurden das kollapsfähige Segment mir der Weichgaumenlänge, der Strecke Hyoid zur Nasallinie und subpalatinale Luftraum mit Hilfe der Korrelationsmatrizen nach Pearson verglichen. Signifikante Korrelationen wurden mit rot markiert, wobei der p-Wert auf 0,05 festgelegt wurde. Es wurden 64 Patienten untersucht.

\begin{tabular}{|l|l|r|r|r|}
\hline Variable & & Weichgaumenlänge & Hyoid-Nasallinie & subpalatinaler Luftraum \\
\hline PNS-Epiglottis & Pearsonkoeffizient & 0,372 & 0,683 & 0,468 \\
\hline & p-Wert & 0,008 & $<0,001$ & 0,001 \\
\hline
\end{tabular}

Das kollapsfähige Segment bei Männern korrelierte mit der Weichgaumenlänge $(\mathrm{p}=0,008)$, dabei nahm die Größe des kollapsfähigen Segmentes mit zunehmender Weichgaumenlänge zu. Gleiches gilt für die Strecke Hyoid zu Nasallinie $(p<0,001)$ und für den subpalatinalen Luftraum $(p=$ 0,001)(Tabelle 2).

\subsubsection{Korrelation der Luftwegsquerschnitte auf unterschiedlichen anatomischen Ebenen}

Um zu untersuchen, ob eine Korrelation der Luftwegsquerschnitte untereinander existiert, wurden die Korrelationsmatrizen nach Pearson angewandt.

Tabelle. 5: Korrelation der Querschnitte des Luftweges für Männer. Es wurden die Luftwegsquerschnitte untereinander mit den Korrelationsmatrizen nach Pearson verglichen. Signifikante Korrelationen wurden mit rot markiert, wobei der p-Wert auf 0,05 festgelegt wurde. Es wurden 64 Patienten untersucht.

\begin{tabular}{|l|l|c|r|r|r|r|}
\hline Variable & & Fläche UPW & Fläche MPW & Fläche Isthmus & Fläche TPW & Fläche LPW \\
\hline Fläche UPW & Pearsonkoeffizient & - & 0,265 & 0,289 & 0,043 & 0,377 \\
\hline & p-Wert & - & 0,034 & 0,021 & 0,737 & 0,002 \\
\hline Fläche MPW & Pearsonkoeffizient & & - & 0,752 & 0,292 & 0,349 \\
\hline & p-Wert & & - & $<0,001$ & 0,018 & 0,004 \\
\hline Fläche Isthmus & Pearsonkoeffizient & & & - & 0,202 & 0,391 \\
\hline & p-Wert & & & - & 0,107 & 0,001 \\
\hline Fläche TPW & Pearsonkoeffizient & & & & - & 0,497 \\
\hline & p-Wert & & & & & - \\
\hline Fläche LPW & Pearsonkoeffizient & & & & & - \\
\hline & p-Wert & & & & & - \\
\hline
\end{tabular}

Die Fläche des Luftweges auf Höhe der harten Gaumenebene (UPW) korrelierte bei Männern mit der Fläche des Luftweges auf Höhe der unteren Inzisivi (MPW) (p=0,034) und der Fläche des 


\section{Ergebnisse}

Luftweges auf Höhe des Isthmus $(p=0,021)$, sowie auf Höhe der Vallecula (LPW) $(p=0,002)$. Mit zunehmender Fläche von UPW nahmen die Flächen von MPW, des Isthmus und von LPW zu.

Weiterhin bestand kein Zusammenhang zwischen der Fläche von UPW und Fläche des Luftweges auf Höhe des dorsalsten Punktes der Zunge (TPW) (Tabelle 3).

Des Weiteren gab es einen Zusammenhang zwischen der Fläche von MPW und des Isthmus $(\mathrm{p}<0,001)$, von TPW $(\mathrm{p}=0,018)$ und von LPW $(\mathrm{p}=0,004)$. Mit zunehmender Fläche von MPW nahmen auch die anderen Flächen zu.

Zwischen der Fläche des Isthmus und von TPW bestand kein signifikanter Zusammenhang. Allerdings bestand zwischen der Fläche des Isthmus und LPW ein Zusammenhang ( $p=0,001)$.

De Luftwegsflächen von TPW und LPW korrelierten miteinander $(\mathrm{p}<0,001)$.

\subsubsection{Korrelation der sagittalen und transversalen Dimensionen des Luftweges}

Um zu untersuchen, ob eine Korrelation zwischen den sagittalen und transversalen Dimensionen des Luftweges existiert, wurden die Korrelationsmatrizen nach Pearson angewandt. Weiterhin wurden überprüft, ob es einen Zusammenhang zwischen den sagittalen Dimensionen untereinander und den transversalen Dimensionen untereinander gibt.

Tabelle 6: Korrelation der sagittalen und transversalen Dimensionen des Luftweges für Männer. Es wurden die sagittalen und transversalen Dimensionen des Luftweges untereinander mit den Korrelationsmatrizen nach Pearson verglichen. Dabei bedeutet a.p. anterior-posterior und trans: transversal. Signifikante Korrelationen wurden mit rot markiert, wobei der p-Wert auf 0,05 festgelegt wurde. Es wurden 64 Patienten untersucht.

\begin{tabular}{|c|c|c|c|c|c|c|c|c|c|c|c|}
\hline Variable & & UPW a.p. & UPW trans & MPW a.p. & MPW trans & Isthmus a.p. & Isthmus trans & TPW a.p. & TPW trans & LPW a.p. & LPW trans \\
\hline \multirow[t]{2}{*}{ UPW a.p. } & Pearsonkoeffizient & - & 0,245 & 0,043 & $-0,007$ & 0,054 & 0,064 & 0,122 & $-0,04$ & 0,297 & 0,333 \\
\hline & p-Wert & - & 0,053 & 0,741 & 0,955 & 0,676 & 0,619 & 0,339 & 0,756 & 0,018 & 0,008 \\
\hline \multirow[t]{2}{*}{ UPW trans } & Pearsonkoeffizient & & - & 0,219 & 0,523 & 0,195 & 0,533 & $-0,134$ & 0,271 & 0,351 & 0,373 \\
\hline & $p$-Wert & & - & 0,084 & $<0,001$ & 0,126 & $<0,001$ & 0,295 & 0,031 & 0,005 & 0,003 \\
\hline \multirow[t]{2}{*}{ MPW a.p. } & Pearsonkoeffizient & & & - & 0,479 & 0,599 & 0,411 & 0,086 & 0,198 & 0,099 & 0,233 \\
\hline & $p$-Wert & & & - & $<0,001$ & $<0,001$ & 0,001 & 0,501 & 0,119 & 0,441 & 0,066 \\
\hline \multirow[t]{2}{*}{ MPW trans } & Pearsonkoeffizient & & & & - & 0,23 & 0,92 & $-0,116$ & 0,568 & 0,277 & 0,262 \\
\hline & $p$-Wert & & & & - & 0,069 & $<0,001$ & 0,364 & $<0,001$ & 0,028 & 0,038 \\
\hline \multirow[t]{2}{*}{ Isthmus a.p. } & Pearsonkoeffizient & & & & & - & 0,339 & 0,231 & 0,11 & 0,234 & 0,229 \\
\hline & $p$-Wert & & & & & - & 0,007 & 0,07 & 0,395 & 0,068 & 0,074 \\
\hline \multirow[t]{2}{*}{\begin{tabular}{|l|} 
Isthmus trans \\
\end{tabular}} & Pearsonkoeffizient & & & & & & - & $-0,128$ & 0,524 & 0,317 & 0,235 \\
\hline & $p$-Wert & & & & & & - & 0,323 & $<0,001$ & 0,012 & 0,066 \\
\hline \multirow[t]{2}{*}{ TPW a.p. } & Pearsonkoeffizient & & & & & & & - & 0,149 & 0,502 & 0,041 \\
\hline & $p$-Wert & & & & & & & - & 0,241 & $<0,001$ & 0,747 \\
\hline \multirow[t]{2}{*}{ TPW trans } & Pearsonkoeffizient & & & & & & & & - & 0,412 & 0,493 \\
\hline & $p$-Wert & & & & & & & & - & 0,001 & $<0,001$ \\
\hline \multirow[t]{2}{*}{ LPW a.p. } & Pearsonkoeffizient & & & & & & & & & - & 0,259 \\
\hline & $p$-Wert & & & & & & & & & - & 0,122 \\
\hline \multirow[t]{2}{*}{ LPW trans } & Pearsonkoeffizient & & & & & & & & & & - \\
\hline & p-Wert & & & & & & & & & & - \\
\hline
\end{tabular}

Der sagittale Durchmesser auf Höhe der harten Gaumenebene (UPW a.p.) korrelierte nur mit dem transversalen und sagittalen Durchmesser auf Höhe der Vallecula (LPW trans mit p=0,008 und LPW a.p. mit p=0,018). Beim transversalen Durchmesser von UPW konnte man einen signifikanten Zusammenhang mit der transversalen Dimension auf Höhe des unteren Inzisivi (MPW trans mit 


\section{Ergebnisse}

$\mathrm{p}<0,001$ ), auf Höhe des Isthmus trans ( $\mathrm{p}<0,001)$, auf Höhe des dorsalsten Punktes der Zunge (TPW trans mit $\mathrm{p}=0,031)$ und auf von LPW trans $(\mathrm{p}=0,003)$ (Tabelle).

Der sagittale Durchmesser auf Höhe vom unteren Inzisivi (MPW a.p.) zeigte eine Korrelation zu dem transversalen Durchmesser vom MPW $(\mathrm{p}<0,001)$, sowie zum sagittalen $(\mathrm{p}<0,001)$ und transversalen $(\mathrm{p}=0,001)$ Durchmesser vom Isthmus. Weiterhin bestand in der transversalen Dimension von MPW ein signifikanter Zusammenhang mit Isthmus trans $(\mathrm{p}<0,001)$, TPW trans ( $\mathrm{p}<0,001)$, LPW a.p. $(\mathrm{p}=0,028)$ und LPW trans $(\mathrm{p}=0,038)$.

Der sagittale und transversale Durchmesser auf Höhe des Isthmus korrelierten miteinander $(\mathrm{p}=0,007)$. Isthmus trans wies einen signifikanten Zusammenhang mit TPW trans $(\mathrm{p}<0,001)$ und LPW a.p. $(\mathrm{p}=0,012)$ auf.

Zwischen TPW a.p. und LPW a.p. $(\mathrm{p}<0,001)$ konnte eine signifikante Korrelation festgestellt werden. Auch zwischen TPW trans und LPW a.p. $(\mathrm{p}=0,001)$ bzw. LPW trans $(\mathrm{p}<0,001)$ besteht ein Zusammenhang.

Bei den aufgeführten signifikanten Zusammenhänge handelt es sich positive Korrelationen.

\subsubsection{Neigung des Unterkieferastes zur mediosagittalen Ebene und Korrelation zum}

\section{Luftwegsquerschnitt}

Um einen signifikanten Zusammenhang zwischen dem Luftweg auf Höhe des unteren Inzisivi (MPW) und der Neigung des Unterkieferastes zur mediosagittalen Ebene (Winkel $(\alpha)$ und Winkel $\beta)$ festzustellen, wurden die Korrelationsmatrizen nach Pearson angewandt.

Tabelle 7: Korrelation der Winkel innen und außen mit dem Luftweg auf Höhe MPW für Männer. Es wurden die Winkel $\alpha$ und $\beta$ mit dem Luftweg auf Höhe MPW mit den Korrelationsmatrizen nach Pearson verglichen. Dabei bedeutet a.p. anterior-posterior und trans: transversal. Signifikante Korrelationen wurden mit rot markiert, wobei der pWert auf 0,05 festgelegt wurde. Es wurden 64 Patienten untersucht.

\begin{tabular}{|l|l|r|r|r|r|}
\hline Variable & & MPW a.p. & MPW trans & Fläche MPW & Isthmus axial \\
\hline Winkel innen & Pearsonkoeffizient & 0,054 & 0,009 & 0,038 & 0,02 \\
\hline & p-Wert & 0,676 & 0,945 & 0,769 & 0,875 \\
\hline Winkel außen & Pearsonkoeffizient & 0,054 & 0,015 & 0,011 & 0,041 \\
\hline & p-Wert & 0,675 & 0,908 & 0,931 & 0,749 \\
\hline
\end{tabular}

Es konnte keine signifikanten Zusammenhänge zwischen den Winkeln $\alpha$ und $\beta$ und dem Luftweg auf Höhe des unteren Inzisivi festgestellt werden (Tabelle). 


\subsection{Korrelation der Messwerte bei Frauen}

\subsubsection{Korrelation des kollapsfähigen Segmentes mit ausgewählten Parametern}

Für die Überprüfung einer Korrelation zwischen dem kollapsfähigen Segment (Strecke PNSEpiglottis) und der Weichgaumenlänge, der Strecke Hyoid zu Nasallinie und der subpalatinale Luftraum wurden die Korrelationsmatrizen nach Pearson angewandt.

Tabelle 8: Korrelation der Strecke PNS-Epiglottis mit ausgewählten Parametern für Frauen. Es wurden das kollapsfähige Segment mir der Weichgaumenlänge, der Strecke Hyoid zur Nasallinie und der subpalatinale Luftraum mit Hilfe der Korrelationsmatrizen nach Pearson verglichen. Signifikante Korrelationen wurden mit rot markiert, wobei der p-Wert auf 0,05 festgelegt wurde. Es wurden 63 Patienten untersucht.

\begin{tabular}{|l|l|r|r|r|}
\hline Variable & & Weichgaumenlänge & Hyoid-Nasallinie & subpalatinaler Luftraum \\
\hline PNS-Epiglottis & Pearsonkoeffizient & 0,372 & 0,746 & 0,275 \\
\hline & p-Wert & $\mathrm{p}=0,003$ & $\mathrm{p}<0,001$ & $\mathrm{p}=0,029$ \\
\hline
\end{tabular}

Das kollapsfähige Segment bei Frauen korrelierte signifikant mit der Weichgaumenlänge $(\mathrm{p}=$ 0,003), dabei nahm die Größe des kollapsfähigen Segmentes mit zunehmender Weichgaumenlänge zu. Gleiches zeigte sich bei der Strecke Hyoid-Nasallinie $(\mathrm{p}<0,001)$ und den subpalatinalen Luftraum $(\mathrm{p}=0,029)$.

\subsubsection{Korrelation der Luftwegsquerschnitte auf unterschiedlichen anatomischen Ebenen}

Um zu untersuchen, ob eine Korrelation der Luftwegsquerschnitten untereinander existiert, wurden die Korrelationsmatrizen nach Pearson angewandt.

Tabelle 9: Korrelation der Querschnitte des Luftweges für Frauen. Es wurden die Luftwegsquerschnitten untereinander mit den Korrelationsmatrizen nach Pearson verglichen. Signifikante Korrelationen wurden mit rot markiert, wobei der p-Wert auf 0,05 festgelegt wurde. Es wurden 65 Patienten untersucht.

\begin{tabular}{|c|c|c|c|c|c|c|}
\hline Variable & & Fläche UPW & Fläche MPW & Fläche Isthmus & Fläche TPW & Fläche LPW \\
\hline \multirow[t]{2}{*}{ Fläche UPW } & Pearsonkoeffizient & - & $-0,02$ & 0,43 & 0,331 & 0,123 \\
\hline & p-Wert & - & 0,877 & $<0,001$ & 0,007 & 0,33 \\
\hline \multirow[t]{2}{*}{ Fläche MPW } & Pearsonkoeffizient & & - & 0,452 & 0,236 & 0,289 \\
\hline & p-Wert & & - & $<0,001$ & 0,058 & 0,019 \\
\hline \multirow[t]{2}{*}{ Fläche Isthmus } & Pearsonkoeffizient & & & - & 0,238 & 0,169 \\
\hline & p-Wert & & & - & 0,056 & 0,178 \\
\hline \multirow[t]{2}{*}{ Fläche TPW } & Pearsonkoeffizient & & & & - & 0,553 \\
\hline & p-Wert & & & & - & $<0,001$ \\
\hline \multirow[t]{2}{*}{ Fläche LPW } & Pearsonkoeffizient & & & & & - \\
\hline & p-Wert & & & & & - \\
\hline
\end{tabular}

Die Fläche des Luftweges auf Höhe der harten Gaumenebene (UPW) korrelierte bei Frauen mit der Fläche des Luftweges auf Höhe des Isthmus $(\mathrm{p}<0,001)$ und der Fläche des Luftweges auf Höhe des 


\section{Ergebnisse}

dorsalsten Punktes der Zunge (TPW) $(\mathrm{p}=0,007)$. Mit zunehmender Fläche von UPW nahmen die Fläche des Isthmus und von TPW zu.

Weiterhin bestand kein Zusammenhang zwischen der Fläche von UPW und der Fläche des Luftweges auf Höhe der unteren Inzisivi (MPW) (p=0,877) und der Fläche auf Höhe der Vallecula von $(\mathrm{LPW})(\mathrm{p}=0,330)$.

Des Weiteren gab es einen Zusammenhang zwischen der Fläche von MPW und des Isthmus ( $\mathrm{p}<0,001)$ und von LPW ( $\mathrm{p}=0,019)$. Mit zunehmender Fläche von MPW nahm die Fläche des Isthmus und von LPW zu. Keinen signifikanter Zusammenhang gab zwischen der Fläche von MPW und von TPW ( $p=0,058)$ sowie zwischen der Fläche des Isthmus und TPW ( $p=0,056)$.

Die Flächen zwischen LPW und TPW korrelierten miteinander ( $\mathrm{p}<0,001)$, allerdings korrelierten die Flächen Isthmus und LPW ( $\mathrm{p}=0,178)$ nicht.

\subsubsection{Korrelation der sagittalen und transversalen Dimensionen des Luftweges}

Um zu untersuchen, ob eine Korrelation zwischen den sagittalen und transversalen Dimensionen des Luftweges existiert, wurden die Korrelationsmatrizen nach Pearson angewandt. Weiterhin wurden überprüft, ob es einen Zusammenhang zwischen den sagittalen Dimensionen untereinander und den transversalen Dimensionen untereinander gibt.

Tabelle 10: Korrelation der sagittalen und transversalen Dimensionen des Luftweges für Frauen. Es wurden die sagittalen und transversalen Dimensionen des Luftweges untereinander mit den Korrelationsmatrizen nach Pearson verglichen. Dabei bedeutet a.p. anterior-posterior und trans: transversal. Signifikante Korrelationen wurden mit rot markiert, wobei der p-Wert auf 0,05 festgelegt wurde. Es wurden 65 Patienten untersucht.

\begin{tabular}{|c|c|c|c|c|c|c|c|c|c|c|c|}
\hline Variable & & UPW a.p. & UPW trans & MPW a.p. & MPW trans & Isthmus a.p. & Isthmus trans & TPW a.p. & TPW trans & LPW a.p. & LPW trans \\
\hline \multirow[t]{2}{*}{ UPW a.p. } & Pearsonkoeffizient & - & 0,47 & $-0,08$ & $-0,02$ & 0,239 & 0,234 & 0,237 & 0,261 & $-0,038$ & 0,053 \\
\hline & $p$-Wert & - & $<0,001$ & 0,526 & 0,877 & 0,055 & 0,061 & 0,057 & 0,036 & 0,766 & 0,676 \\
\hline \multirow[t]{2}{*}{ UPW trans } & Pearsonkoeffizient & & - & $-0,118$ & 0,243 & 0,238 & 0,378 & 0,141 & 0,307 & 0,014 & 0,103 \\
\hline & p-Wert & & - & 0,348 & 0,051 & 0,056 & 0,002 & 0,263 & 0,013 & 0,912 & 0,413 \\
\hline \multirow[t]{2}{*}{ MPW a.p. } & Pearsonkoeffizient & & & - & 0,146 & 0,414 & 0,044 & 0,291 & $-0,026$ & 0,259 & 0,024 \\
\hline & p-Wert & & & - & 0,248 & 0,001 & 0,727 & 0,02 & 0,839 & 0,039 & 0,849 \\
\hline \multirow[t]{2}{*}{ MPW trans } & Pearsonkoeffizient & & & & - & 0,222 & 0,737 & $-0,028$ & 0,509 & 0,203 & 0,261 \\
\hline & p-Wert & & & & - & 0,077 & $<0,001$ & 0,827 & $<0,001$ & 0,108 & 0,038 \\
\hline \multirow[t]{2}{*}{ Isthmus a.p. } & Pearsonkoeffizient & & & & & - & 0,429 & 0,332 & 0,124 & 0,143 & $-0,024$ \\
\hline & $p$-Wert & & & & & - & $<0,001$ & 0,008 & 0,331 & 0,264 & 0,85 \\
\hline \multirow[t]{2}{*}{ Isthmus trans } & Pearsonkoeffizient & & & & & & - & $-0,064$ & 0,433 & 0,098 & 0,299 \\
\hline & $p$-Wert & & & & & & - & 0,62 & $<0,001$ & 0,445 & 0,017 \\
\hline \multirow[t]{2}{*}{ TPW a.p. } & Pearsonkoeffizient & & & & & & & - & 0,439 & 0,472 & 0,141 \\
\hline & p-Wert & & & & & & & - & $<0,001$ & $<0,001$ & 0,262 \\
\hline \multirow[t]{2}{*}{ TPW trans } & Pearsonkoeffizient & & & & & & & & - & 0,435 & 0,467 \\
\hline & p-Wert & & & & & & & & - & $<0,001$ & $<0,001$ \\
\hline \multirow[t]{2}{*}{ LPW a.p. } & Pearsonkoeffizient & & & & & & & & & - & 0,344 \\
\hline & $p$-Wert & & & & & & & & & - & 0,008 \\
\hline \multirow[t]{2}{*}{ LPW trans } & Pearsonkoeffizient & & & & & & & & & & - \\
\hline & p-Wert & & & & & & & & & & - \\
\hline
\end{tabular}

Der sagittale Durchmesser auf Höhe der harten Gaumenebene (UPW a.p.) korrelierte nur mit dem transversalen Durchmesser von UPW $(\mathrm{p}<0,001)$ und auf Höhe des dorsalsten Punktes der Zunge (TPW trans mit $\mathrm{p}=0,036$ ). Beim transversalen Durchmesser von UPW konnte man einen 


\section{Ergebnisse}

signifikanten Zusammenhang mit der transversalen Dimension auf Höhe des Isthmus trans $(\mathrm{p}=0,002)$ und TPW trans $(\mathrm{p}=0,013)$ (Tabelle).

Der sagittale Durchmesser auf Höhe vom unteren Inzisivi (MPW a.p.) zeigte eine Korrelation zu zum sagittalen Durchmesser vom Isthmus $(\mathrm{p}=0,001)$, von TPW $(\mathrm{p}=0,020)$ und LPW $(\mathrm{p}=0,039)$. Weiterhin bestand in der transversalen Dimension von MPW ein signifikanter Zusammenhang mit Isthmus trans $(\mathrm{p}<0,001)$, TPW trans $(\mathrm{p}<0,001)$ und LPW trans $(\mathrm{p}=0,038)$.

Der sagittale Durchmesser auf Höhe des Isthmus korrelierte mit Isthmus trans $(\mathrm{p}<0,001)$ und TPW a.p. $(\mathrm{p}=0,008)$. Isthmus trans wies einen signifikanten Zusammenhang mit TPW trans $(\mathrm{p}<0,001)$ und LPW trans $(\mathrm{p}=0,017)$ auf.

Zwischen TPW a.p. und TPW trans $(\mathrm{p}<0,001)$ bzw. LPW a.p. $(\mathrm{p}<0,001)$ konnte eine signifikante Korrelation festgestellt werden. Auch zwischen TPW trans und LPW a.p. $(p<0,001)$ bzw. LPW trans ( $<<0,001)$ besteht ein Zusammenhang. Weiterhin korrelierten beide Dimensionen auf Höhe LPW miteinander $(\mathrm{p}=0,008)$.

Bei den aufgeführten signifikanten Zusammenhänge handelt es sich positive Korrelationen.

\subsubsection{Neigung des Unterkieferastes zur mediosagittalen Ebene und Korrelation zum}

\section{Luftwegsquerschnitt}

Um einen signifikanten Zusammenhang zwischen dem Luftweg auf Höhe des unteren Inzisivi (MPW) und der Neigung des Unterkieferastes zur mediosagittalen Ebene (Winkel $(\alpha)$ und Winkel $\beta)$ festzustellen, wurden die Korrelationsmatrizen nach Pearson angewandt.

Tabelle 11: Korrelation der Winkel $\alpha$ und $\beta$ mit dem Luftweg auf Höhe MPW für Frauen. Es wurden die Winkel $\alpha$ und $\beta$ mit dem Luftweg auf Höhe MPW mit den Korrelationsmatrizen nach Pearson verglichen. Dabei bedeutet a.p. anterior-posterior und trans: transversal. Signifikante Korrelationen wurden mit rot markiert, wobei der p-Wert auf 0,05 festgelegt wurde. Es wurden 65 Patienten untersucht.

\begin{tabular}{|l|l|r|r|r|r|}
\hline Variable & & MPW a.p. & MPW trans & Fläche MPW & Isthmus axial \\
\hline Winkel innen & Pearsonkoeffizient & $-0,036$ & $-0,129$ & $-0,071$ & 0,217 \\
\hline & p-Wert & 0,779 & 0,308 & 0,579 & 0,084 \\
\hline Winkel außen & Pearsonkoeffizient & 0,013 & $-0,15$ & $-0,039$ & 0,33 \\
\hline & p-Wert & 0,92 & 0,238 & 0,76 & 0,008 \\
\hline
\end{tabular}

Es konnte keine signifikanten Zusammenhänge zwischen den Winkel $\alpha$ und dem Luftweg auf Höhe des unteren Inzisivi festgestellt werden (Tabelle 11). Eine signifikante Korrelation bestand zwischen dem Winkel $\beta$ und dem Isthmus axial ( $\mathrm{p}=0,008)$. 


\section{Diskussion}

\section{Diskussion}

Zur Anatomie des orofazialen Systems gibt es verschiedene Studien. Die objektive Untersuchung des Pharynx und angrenzender Strukturen wurde mit einer Reihe radiologischer, bildgebender Verfahren möglich. Im überwiegenden Teil der Studien erfolgte die Durchführung mit Hilfe des Fernröntgenseitenbildes (Partinen et al. 1988, Hochban et al 1994, Tangugsorn et al. 1995, Pracharktam et al. 1996, Solow et al. 1996, Battagel und L'Estrange 1996, Pradel et al. 2000, Battagel et al. 2000, Battagel et al. 2002, Johal et al. 2007, Oosterkamp et al. 2007). Des Weiteren stehen Schichtbildverfahren wie Computertomographie (Avrahami und Englender 1995, Avrahami et al. 1996, Pepin et al. 1999, Fajdiga 2005, Yucel et al. 2005, Fairburn et al. 2007, Koren et al. 2009, Van Holsbeke et al. 2013) und Magnetresonanztomographie (Horner et al. 1989 und Hora et al. 2007) zur Verfügung. Durch diese Studien wurden weitreichende Erkenntnisse über das orofaziale System gewonnen. Der Vergleich der Ergebnisse mit denen aus anderen Studien gestaltete sich schwierig, da die Aufnahmebedingungen, Messstrecken und Bezugsebenen variierten. Bisher konnten Zahnmediziner nur mit Hilfe des Fernröntgenseitenbildes den Pharynx zweidimensional bildlich betrachten. Mit dem DVT ist es seit 1997 möglich, dreidimensionale Bilder des Pharynx zu erstellen. Dies stellt für den behandelnden Zahnarzt eine deutliche Erweiterung seiner radiologischen Möglichkeiten dar (Ziegler et al. 2002).

Da Durchführung und Befundung dieses neuen Verfahrens durch die zahnärztlichen Behandler selbst erfolgen kann, hat die DVT in den letzten Jahren zunehmende Verbreitung gefunden (Danforth 2003). Aus diesem Grund wurde das DVT für die Bestimmung der Weich- und Hartgewebe im Bereich des oberen Luftweges und der Mundhöhle in horizontaler Körperposition, für die Darstellung von geschlechtsspezifischen Unterschieden im Bereich des oberen Luftweges sowie für die Beschreibung der Anordnung des aufsteigenden Astes des Unterkiefers in der horizontaler Ebene für diese Studie gewählt. Die Haupteinsatzgebiete sind weiterhin die prächirurgischen Abklärungen bei Zysten, bei Kieferhöhlenveränderungen, bei Weisheitszahnextraktionen sowie zur präimplantorische Planung. 
4.1. Kritische Betrachtung der angewandten Methode

\subsubsection{Aspekte der Patientenauswahl}

Die Patientenauswahl erfolgte nach dem Geschlecht, dem Alter und den darzustellenden anatomischen Strukturen. Beim Geschlecht kam es zufällig zu einer gleichmäßigen Verteilung des Patientenkollektivs. Insgesamt wurden 129 Patienten ausgewählt, wobei es sich um 64 Männer und 65 Frauen handelte. Bei dem Alter erfolgte die Einschränkung auf die Patientengruppe zwischen 30 und 68 Jahre. Das Durchschnittsalter lag für die Frauen bei 45,63 Jahre \pm 11,12 und für die Männer bei 45,83 Jahre $\pm 11,16$, was den Vergleich beider Geschlechter möglich machte. Das Patientenkollektiv war nur eingeschränkt repräsentativ, da nur Patienten zwischen 30 und 68 Jahren, mit gesicherter Okklusion, mit den Schneidezähnen und mit dem nötigen Aufnahmefeld ausgewählt wurden. Nicht alle anatomischen Strukturen des Pharynx konnten dargestellt werden, wodurch ich bei einzelnen Patienten auf einige Messpunkte und -strecken verzichten musste.

In Bezug auf das Patientenalter lag die vorliegende Studie im Vergleich zu Tangugsorn et al. (1995) (36,8 Jahre im Alter zwischen 28 und 45 Jahren) und zu Abramson et al. (2009) (33,7 Jahre $\pm 14,8$ ) mit dem Durchschnittsalter deutlich über den Ergebnissen, gegenüber Solow et al. (1996) (50 Jahre \pm 9,4 Stdabw. im Alter zwischen 29 und 70 Jahre), Lowe et al. (1986) (48 Jahre \pm 11 ) und Johal et al. (2007) (48,6 Jahre \pm 8,3 im Alter zwischen 27 und 66 Jahren) leicht unterhalb der genannten Studien. Da die knöchernen Strukturen bei Erwachsenen altersunabhängig sind, hat das unterschiedliche Alter keine Auswirkung auf den Vergleich der Studien. Auch die Weichteilstrukturen hängen mehr vom Gewicht der Probanden ab als vom Alter. Inwieweit das Gewicht Einfluss auf die Anatomie nimmt, müssen andere Studien klären, da hier kein Augenmerk darauf gelegt werden konnte.

Bei der Kollektivgröße gab es in den Studien zum Teil große Unterschiede, was für die Repräsentationsfähigkeit der Ergebnisse in Bezug auf die Gesamtbevölkerung Einfluss hatte, d.h. je größer die Patientenkollektive waren, desto größer war deren statistische Aussagekraft. Im Vergleich zu Abramson et al. (2009) mit 32 Patienten, Johal et al. (2007) mit 99 Patienten, Fajdiga (2005) mit 14 Patienten, Battagel et al. (2000) mit 24 Patienten und Tangugsorn et al. (1995) mit insgesamt 36 Patienten, hatte diese Studie DVT-Datensätze von 129 Patienten ausgewertet.

Ein weiterer prägnanter Aspekt der Studie ist der Frauenanteil. Dieser unterscheidet sich erheblich von dem in anderen Studien, in denen man entweder keinen geschlechtsspezifischen Unterschied machte oder erst gar keine Frauen in das Patientenkollektiv aufnahm. Dies 


\section{Diskussion}

erschwerte einen Vergleich der erhobenen Messergebnisse mit anderen Studien. Im Vergleich zu Abramson et al. (2009) mit 11 Frauen, bei Johal et al. (2007) mit 21 Frauen und bei Ingman et al. (2004) mit 12 Frauen wurden in dieser Studie 65 Frauen untersucht. Auf Grund des wesentlich höheren Frauenanteils kann eine repräsentativere Aussage über die Anatomie des oropharyngealen Luftweges bei den Frauen und dementsprechend Gemeinsamkeiten bzw. Unterschiede zwischen den Geschlechtern getroffen werden.

Des Weiteren wurden die Ergebnisse aus dieser Studie mit Patientenkollektiven verglichen, bei denen OSAS ausgeschlossen werden konnte. Für die vorliegende Studie war eine OSASErkrankung kein Ausschlusskriterium. Dementsprechend flossen die Ergebnisse der Vermessung von erkrankten Personen mit in die Auswertung ein. Im Gegensatz zu den anderen Studien, welche die Patienten in Gesunde und Erkrankte einteilten, handelt es sich hier um Durchschnittswerte für die Gesamtbevölkerung. Dies macht allerdings einen Vergleich der Messwerte zwischen den verschiedenen Studien, vor allem bei denen mit obstruktivem Schlafapnoesyndrom, schwierig.

\subsubsection{Aufnahmebedingungen}

Um Projektionsfehler bei den Aufnahmen zu verringern, sind konkrete Anweisungen der RöntgenTechnischen-Angestellten wichtig. Dabei sollten die Patienten Okklusionskontakt aufweisen (Tangugsorn et al. 1995, Pracharktam et al. 1996 und Johal et al. 2007) und vor der Aufnahme geschluckt haben (Lowe et al. 1985, Solow et al. 1996, Pepin et al. 1999 und Oosterkamp et al. 2007), damit in der Mundhöhle ein negativer Druck entsteht und es somit zu einer Anlagerung der Zunge an dem Gaumen kommt. Trotz der Instruktion durch das Personal platzierten gelegentlich die Patienten die Zunge nur im vorderen Bereich an dem Gaumen. Gründe für die falsche Positionierung konnten zum Beispiel sein, dass die Patienten die Anweisungen falsch verstanden haben oder die Aufrechterhaltung des Unterdruckes während der gesamten Aufnahmezeit von 76 Sekunden seitens der Versuchspersonen nicht möglich war. Es bestand dadurch die Möglichkeit, dass durch Nichtbefolgen der anatomischen Ausgangsstellung eine verfälschte Vermessung erfolgte. Um eine sichere Positionierung der Zunge an den Gaumen zu gewährleisten, wäre die Nutzung des Zungenrepositionierungsmanöver sinnvoll. Dabei kann man durch eine Membrantrichterplatte den intraokklusalen Druck beobachten. Demzufolge kann man die Erzielung eines Unterdruckes kontrollieren und damit indirekt eine Aussage zur Zungenlage am Gaumen treffen (Sömmer 2008).

Für die DVT-Aufnahmen wurde eine reproduzierbare Position der Patienten gewählt. Die Patienten lagen auf dem Rücken, den Kopf in einer Schale positioniert. Bei anderen Studien, vor allem bei denen über das Fernröntgenseitenbild, wurde eine natürliche aufrechte Kopfposition (Lowe et al. 


\section{Diskussion}

1985, Tangugsorn et al. 1995, Solow et al. 1996, Pracharktam et al. 1996, Johal et al. 2007) oder eine Kopfposition im Cephalostat (Hochban et al. 1994, Partinen et al. 1988, Pradel et al. 2000, Battagel et al. 2000) gewählt. Bei den CT-Studien von Bohlman et al. (1983), Galvin et al. (1989), Avrahami und Englender (1995), Pepin et al. (1999), Peh et al. (2000), Fajdiga (2005), Yucel et al. (2005), Fairburn et al. (2007) und Koren et al. (2009) ist die Lagerung ähnlich wie bei den DVTAufnahmen, sodass die Messwerte vergleichbar sind. Gleiches gilt auch für die MRT-Studien von Baik et al. (2002) und Hora et al. (2007).

\subsubsection{Technische Aspekte der Digitalen Volumentomographie}

Wie im Methodikkapitel bereits erwähnt wird ein anatomisches Volumen mit den Abmessungen $15 \times 15 \mathrm{~cm}$ in Zylinderform abgebildet. Dementsprechend war es nicht möglich alle DVTAufnahmen, die $\mathrm{zu}$ Verfügung standen, für diese Studie $\mathrm{zu}$ verwenden. Gerade bei Kieferhöhlenaufnahmen, der Orbita und auch bei Aufnahmen des Unterkiefers konnte nicht der gesamte Pharynx dargestellt werden. Auch die gleichzeitige Darstellung des Hyoids, der Vallecula glossoepiglottica und der Nasallinie war nicht immer gegeben, sodass eine nicht geringe Anzahl an Probandenaufnahmen nicht genutzt werden konnte. Auch Van Holsbeke et al. (2013) bemängelt, dass das Aufnahmefeld für die gleichzeitige Abbildung von Nasalregion und oberen Luftweg in einem einzigen Scan $\mathrm{zu}$ klein ist. $\mathrm{Da}$ bei der Computertomografie und bei der Magnetresonanztomografie das Nutzstrahlenfeld individuell angepasst werden kann, besteht diese Einschränkung hier nicht. Gleiches gilt auch für das Fernröntgenseitenbild. Insgesamt musste aufgrund des kleinen Aufnahmefeldes auf die Vermessung der Strecke Hyoid zur Nasallinie in 16 von 129 Fällen und die Luftwegsvermessung auf Höhe der Vallecula in 35 von 129 Fällen verzichtet werden. Hinsichtlich der Strecke Nasallinie zum Zungenbein sind dadurch die Messergebnisse kritisch zu betrachten. Es wurde ein Mittelwert für die Männer von 70,63 mm gemessen. Wären auch tiefer liegendere Zungenbeine messbar gewesen, hätte sich der Mittelwert deutlich erhöht.

Aufgrund der relativ langen Aufnahmedauer von 76 Sekunden bei einer effektiven Expositionszeit von 18 Sekunden ließen sich Bewegungsunschärfen nicht vermeiden. Gerade bei Kopfbewegungen und beim Ein- und Ausatmen traten diese auf. Durch die technische Weiterentwicklung der Digitalen Volumentomographie konnte eine Verringerung der Aufnahmedauer erreicht werden. Dadurch war eine Minimierung der Bewegungsunschärfen möglich, was zu einer Verbesserung der Pharynxvermessung führte. Cacaci et al. (2007) stellten eine Übersicht mit neueren Digitalen Volumentomographen zusammen. Daraus war ersichtlich, dass schon heute entsprechende Geräte existieren, die wesentlich geringere Aufnahmezeiten benötigen. Genannt seien an dieser Stelle das 


\section{Diskussion}

3D Accuitomo FPD von J. Morita mit 17,5 Sekunden, das Galileos von Sirona Dental mit 14 Sekunden, das 3D eXam von KaVo mit 8,5 Sekunden und das NewTom VG von NewTom mit 36 Sekunden. Demzufolge sind die neuen Digitalen Volumentomographen schneller, allerdings werden die Patienten im Sitzen bzw. im Stehen untersucht, d.h. man kann mit den neueren Geräten keine Aussage über den Luftweg in horizontaler Lage treffen.

Die zurzeit verwendete Software NewTom Version 3.37 erlaubt noch keine Flächenberechnung. Um trotzdem eine Aussage über die Dimension des Luftweges zu ermöglichen, nutzte diese Studie die anterior-posterioren und transversalen Strecken zur Berechnung der Flächen. Der Luftweg wurde in dieser Studie als Ellipse angenommen und mit der entsprechenden Formel berechnet. Diese Methode liefert aber nur einen Näherungswert. Bohlman et al. (1983), Peh et al. (2000), Fajdiga (2005) und Koren et al. (2009) haben mittels CT mit entsprechenden Programmen die Flächen des Luftweges exakter messen können.

Zusätzlich gibt es auch beim Luft-Weichgewebskontrast Unterschiede zwischen DVT und Fernröntgenseitenbild. Beim DVT ist er ausreichend groß ist und man konnte im Vergleich zu den Fernröntgenseitenbildstudien von Battagel und L`Estrange (1996), Pracharktam et al. (1996), Battagel et al. (2000) und Johal et al. (2007) auf Bariumsulfat als Kontrastmittel verzichten. Lowe et al. (1986) benutzten „Microtrast Oesophageal Cream“, um die Außenflächen von Zunge und den pharyngealen Weichgeweben gegenüber dem Luftweg besser unterscheiden zu können. Ein großer Nachteil des DVTs gegenüber dem CT und MRT ist der fehlende Weichgewebskontrast, d.h. man kann verschiedene Weichgewebe nicht voneinander unterscheiden Dies erschwerte die Identifizierung von Messpunkten. Besteht Kontakt zweier Weichgewebe, hatte dies zur Folge, dass die Begrenzungen der jeweiligen Gewebe im Kontaktflächenbereich nicht zu erkennen waren. Das zeigte sich z.B. bei dem Kontakt des weichen Gaumens mit der Zunge, was die Vermessung der Gaumenbreite erschwerte. Aus diesem Grund musste in 62 von 129 Fällen auf eine Vermessung verzichtet werden. Auch wenn die Epiglottis am Zungenrücken anlag, war deren Spitze nicht eindeutig zu identifizieren (siehe Fallbeispiele 7.4.). Auch Ziegler et al. (2002) und Holberg et al. (2005) gaben an, dass sich das DVT nur sehr eingeschränkt zur Weichgewebsdiagnostik eignet.

Das DVT eignet sich für die Hartgewebsunterscheidung. Die Studie von Valiyaparambil et al. (2012) zeigte, dass moderne DVTs in der Lage sind verschiedene Knochenqualitäten zu unterscheiden. Dies ist möglich, weil die Grauwerte mit dem Calciumgehalt des Knochens linear ansteigen. Auch lassen sich Hartgewebe (Knochen und Zähne) und Weichgewebe im DVT gut voneinander unterscheiden, wodurch Messfehler vermieden werden können.

Trotz der Tatsache, dass es mit der Digitalen Volumentomographie nicht möglich ist, zwischen verschiedenen Weichgewebsstrukturen zu unterscheiden, sind die Grenzen zwischen Weichgewebe und Luftraum darstellbar. Diese Eigenschaften qualifizieren die Digitale Volumentomographie zum 


\section{Diskussion}

diagnostischen Verfahren mit hohem Potenzial in Bezug auf die Beseitigung der bisher problematischen dreidimensionalen Auswertung des Luftweges.

\subsubsection{Auswahl der Messparameter}

Einer der wichtigsten Aspekte bei der Erstellung von deskripten Studien ist die gesicherte Reproduzierbarkeit der Messergebnisse. Fehler bei der Lokalisation der zu vermessenden anatomischen Strukturen in den DVT-Datensätzen beeinflussen die Reproduzierbarkeit. Das nicht eindeutige Erkennen einzelner anatomischer Referenzpunkte ist somit die häufigste Ursache für einen willkürlichen Messfehler (Housten 1983). Daher erfolgte die Vermessung erst nach entsprechenden Übungen und Probeauswertungen der DVT-Daten durch eine Person. Dadurch konnte die interindividuelle Fehlerbreite minimiert werden.

Die Nutzung der Nasallinie (Verbindungslinie zwischen den Punkten Spina nasalis anterior und posterior) als Referenzebene steigerte die Reproduzierbarkeit, wodurch ein exakter Vergleich der Messwerte zwischen den Patienten möglich wurde.Die Referenzebene ist eindeutig und auch bei zahnlosen Patienten zu erkennen. Aus dem dreidimensionalen Datensatz werden die 1,0 mm dicken Axialschnitte parallel zur Nasallinie berechnet, sodass auch dort reproduzierbare und vergleichbare Schnitte entstehen, um den Luftweg zu vermessen. Andere Studien messen nicht parallel zu einer Referenzebene, sondern die geringsten sagittalen Abstände zwischen der Pharynxhinterwand und bestimmten Punkten, wie z.B. der Uvulaspitze (Hochban et al. 1994, Tangugsorn et al. 1995, Solow et al. 1996, Battagel und L'Estrange 1996, Pradel et al. 2000, Oosterkamp et al. 2007), der kleinsten Stelle hinter dem weichen Gaumen (Isthmus) (Solow et al. 1996, Battagel et al. 2000, Johal et al. 2007, Teitelbaum et al. 2007), hinter der Zunge (Solow et al. 1996, Battagel und L'Estrange 1996, Battagel et al. 2000, Johal et al. 2007, Teitelbaum et al. 2007) oder der Vallecula (Hochban et al. 1994, Tangugsorn et al. 1995, Solow et al. 1996, Oosterkamp et al. 2007). Nachteil dieser Methoden ist, dass zwei Messpunkte pro Messstrecke bestimmt werden müssen, was eine erhöhte Fehleranfälligkeit hat und die Reproduzierbarkeit negativ beeinflusst. Die in dieser Studie verwendete Parallelmessmethode der Referenzebene ermöglichte eine Standardisierung innerhalb der Datenerhebung, da lediglich die Bestimmung des anterioren Punktes nötig war. Hierdurch ergibt sich der wesentliche Vorteil der Vergleichbarkeit aller erhobenen Punktmessdaten der verschieden Patienten. Der posteriore Punkt an der hinteren Pharynxwand wurde durch die Nasallinie als Referenzebene vorgegeben. Ein Nachteil bei der Vermessung parallel zur Nasallinie war, dass die Messungen nicht rechtwinklig zum Luftweg erfolgten und sich dadurch größere Strecken in anterior-posteriorer Richtung ergaben. Lowe et al. (1985), Hochban et al. (1994), und Pradel et al. (2000) benutzten Hilfslinien (Mandibularlinie oder Okklusionsebene), um auf deren Verlängerung 


\section{Diskussion}

den Pharynx zu vermessen. Aufgrund der Abhängigkeit der Mandibularlinie und Okklusionsebene von der Bezahnung, Kopfhaltung und Mundöffnung des Patienten, sind die Messpunkte nicht auf andere Patienten übertragbar.

Allerdings musste auch die vorliegende Studie auf einen schlecht reproduzierbaren Messpunkt zurückgreifen. Es handelt sich dabei um den unteren Inzisialpunkt. Dieser ist zwar gut zu identifizieren, aber er ist abhängig von der Mundöffnung und der Lage des Unterkiefers. Im Vergleich zur Lage bei Interkuspitation liegt der Inzisialpunkt je nach Mundöffnung weiter kaudal und je nach Grad der Rückverlagerung des Unterkiefers weiter dorsal, was gleichzeitig eine Rückverlagerung der Zunge nach dorsal bedeutet. Dementsprechend würde es zu einer Verkleinerung der intermaxillären Raumlänge, des anterior-posterioren Durchmessers auf Höhe MPW und TPW kommen, was folgernd auch zu einer Verkleinerung der Fläche des Luftweges führen würde. Des Weiteren kann der Inzisialpunkt auf Grund von ausgedehnten Abrasionen der Schneidekante verfälscht sein. Als sinnvolle Alternative zum unteren Inzisialpunkt wäre die Verwendung des oberen Inzisialpunktes. Dieser ist unabhängig von der Mundöffnung und auch von der Lage des Unterkiefers.

\subsection{Anatomischer Vergleich zwischen den Geschlechtern}

Die vorliegende Arbeit zeigt, dass es eine Reihe von verschiedenen anatomischen Strecken und Flächen gibt, die sich signifikant zwischen den Geschlechtern unterscheiden. Dies sind nicht nur Messwerte, die die Größenunterschiede (Mittel- und Untergesicht) zwischen Männern und Frauen widerspiegeln. Es ist bekannt, dass Männer ein größeres Mittel- und Untergesicht haben. Dementsprechend weist das männliche Geschlecht auch einen signifikant längeren harten Gaumen (Männer: 56,64 mm, Frauen: 52,72 mm), sowie einen größeren Abstand zwischen den lingualen Höckern der Unterkiefers (Männer: 35,96 mm, Frauen: 33,35 mm) und eine erhöhte intermaxilläre Raumlänge auf (Männer:80,14 mm, Frauen: 76,08 mm). Demzufolge war zu erwarten, dass es auch signifikante Unterschiede in der Dimension des Weichgewebes und des Luftweges zwischen den Geschlechtern gibt. Diese Studie kann aber zu dem Ergebnis, dass nicht alle Messstrecken und Flächen geschlechtsspezifische Unterschiede aufweisen. Bei den Männern zeigte sich ein signifikant längerer und breiterer weicher Gaumen (Männer: 41,93 mm und 10,83 mm, Frauen: 35,99 mm und 9,31 mm), ein längeres kollapsfähiges Segment (PNSEpiglottisspitze) (Männer: 59,52 mm, Frauen: 50,38 mm), eine längere Strecke Nasallinie zu dem Unterkiefer (Männer: 62,04 mm, Frauen: 58,24 mm) sowie eine größere Abstand von Hyoid zur Nasallinie (Männer: 70,63 mm, Frauen: 56,97 mm). Auch diese Werte spiegeln die zu erwarteten Größenunterschiede zwischen den Geschlechtern wider. 


\section{Diskussion}

Gemeinsamkeiten zwischen Männer und Frauen gab es bei Kontaktstrecke zwischen weichen Gaumen und Zunge (Männer: 21,84 mm, Frauen: 20,09 mm), bei dem subpalatinalen Luftraum

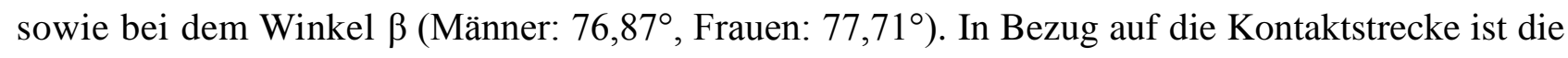
Gemeinsamkeit verwunderlich. Man hätte erwartet, dass die Kontaktstrecke bei Männern größer ist, weil der weiche Gaumen der Männer deutlich größer ist als bei Frauen. Dementsprechend liegt die Zunge bei Frauen im Verhältnis zur Weichgaumenlänge mehr am weichen Gaumen an. Damit könnte die Zunge bei Frauen den Gaumen mehr stabilisieren als bei Männern.

Im Gegensatz zu den oben aufgeführten Messstrecken waren die Ergebnisse für den oberen Luftweg uneinheitlich. Während die Männer im Hypopharynx (LPW) und im Bereich des Zungenrückens (TPW) einen größeren anterior-posterioren Durchmesser (Männer: 14,46 mm und 11,99 mm, Frauen: 11,55 $\mathrm{mm}$ und 10,14 mm) aufweisen, war dieser auf Höhe des unteren Inzisialpunktes (MPW) bei Frauen deutlich größer (Männer: 8,14 mm, Frauen: 9,24 mm). Dies könnte man damit erklären, dass die Männer eine größere Weichgaumenbreite haben und demzufolge diese den oberen Luftweg hinter dem Velum verkleinert. Betrachtet man allerdings die kleinsten anterior-posterioren Durchmesser in der axialen (Isthmus axial) und mediosagittalen Betrachtung (Isthmus), wird ersichtlich, dass es keinen signifikanten Unterschied zwischen den beiden Geschlechtern gibt (Männer: 4,28 mm, Frauen: 4,62 mm und Männer: 6,52 mm und Frauen: 6,46 mm). Dementsprechend werden die neuromuskulären Prozesse des Pharynx den Luftweg in bestimmten Umfang offen halten, sodass der Durchmesser nicht unter ein bestimmtes Minimum fällt. Dieses Minimum ist bei beiden Geschlechtern annähernd gleich und hat wahrscheinlich nichts mit den Ausmaßen des Mittelund Untergesichtes zu tun. Das ist erstaunlich, weil man erwartet hätte, dass der Isthmus bei den Männern aufgrund des dickeren Gaumens kleiner ist als bei den Frauen. Ein Grund dafür könnte sein, dass sich bei den Männern das Gaumensegel nach anterior verdickt und damit der Luftweg unabhängig von der Dicke des Gaumensegels ist. Der Isthmus befindet sich größtenteils hinter dem Velum, selten hinter dem Zungenrücken. Der Abstand vom Isthmus zur harten Gaumenebene war beim männlichen Geschlecht $(32,47 \mathrm{~mm})$ deutlich größer als beim weiblichen $(26,69 \mathrm{~mm})$. Grund dafür ist, dass das Mittelgesicht und damit verbunden der weiche Gaumen bei den Männern deutlich länger ist als bei den Frauen. Des Weiteren ist der Isthmus auch eher im Bereich der Uvulaspitze $\mathrm{zu}$ finden als in der Velummitte. Dementsprechend wirkt sich die Länge des Velums auch auf die Position des Isthmus aus. Dies hat zur Folge, dass je länger das Velum ist desto größer ist der Abstand vom Isthmus zur harten Gaumenebene.

Überraschende Ergebnisse lieferte die Vermessung des oberen Luftweges in der transversalen Dimension. Es konnte kein transversalen Unterschied zwischen den beiden Geschlechtern 


\section{Diskussion}

weder im Bereich des Epi- (UPW) und Mesopharynx (MPW, Isthmus und TPW) noch im Bereich des Hypopharynx (LPW) ermittelt werden. Dementsprechend ist der obere Luftweg in Bezug auf seine transversale Ausdehnung geschlechtsunspezifisch. Es wäre aber zu erwarten gewesen, dass Männer aufgrund des größeren Halsumfangs auch einen größeren transversalen Luftwegsdurchmesser aufweisen. Der dickere Hals wird offensichtlich nur durch die Zunahme des Weichteilgewebes hervorgerufen. Schon 1999 stellten Whittle et al. fest, dass Männer einen größeren Volumenanteil an Weichgewebe in der Halsgegend haben, was den größeren Halsumfang bei annähernd gleichen transversalen Luftwegsdimensionen gegenüber Frauen erklären könnte

Die Unterschiede und Gemeinsamkeiten zwischen den Geschlechtern sind nicht nur auf den anterior-posterioren und transversalen Durchmessern beschränkt, sondern sind auch in den Luftwegsflächen erkennbar.. Da sich die Luftwegsflächen aus den sagittalen und transversalen Messwerten zusammensetzen, verwundert es nicht, dass sich bei der Berechnung der Flächen Unterschiede ergeben, da bereits unterschiedliche Durchmesser im sagittalen Bereich ermittelt worden. Insgesamt waren die Luftwegsflächen im Bereich des Hypopharynx (LPW) und des Zungenrückens (TPW) bei den Männern signifikant größer, was das größere Mittel- und Untergesicht widerspiegelt. Auf Höhe des unteren Inzisialpunktes (MPW) war der Luftweg bei den Frauen größer. Grund könnte zum einen die kleinere Weichgaumenbreite sein, zum anderen die größere Kontaktstrecke des weichen Gaumens mit der Zunge im Verhältnis zur Weichgaumenlänge. Gemeinsamkeiten gab es im Bereich des Isthmus und auf Höhe des harten Gaumens (UPW).

Vergleicht man die Luftwegsflächen untereinander, würde man erwarten, dass die Flächen miteinander korrelieren. Das war auch zum großen Teilen der Fall. Allerdings gab es bei den Männern keine Korrelation zwischen der Fläche UPW und der Fläche TPW, sowie zwischen der Fläche Isthmus und der Fläche TPW. Demzufolge sind die Flächen unabhängig voneinander. Bei den Frauen waren mehrere Flächen unabhängig voneinander, v.a. die Flächen LPW und TPW korrelierten kaum. Fläche LPW wies keine Korrelation mit Fläche UPW und Fläche Isthmus auf, bei Fläche TPW konnte kein Zusammenhang mit dem Fläche Isthmus und Fläche MPW festgestellt werden. Auch Fläche MPW und Fläche UPW waren voneinander unabhängig. Auch bei den sagittalen und transversalen Messwerten zeigt sich beiden Geschlechtern ein uneinheitliches Ergebnis. Während der Epipharynx in der sagittalen Ausdehnung mit kaum einer anderen Luftwegsdimension korreliert, gab es Zusammenhänge zwischen der transversaler Ausdehnung des Epipharynx und den transversalen Dimensionen des Meso- und Hypopharynx. Demzufolge ist die transversale Dimension des Epipharynx unabhängig von den sagittalen Dimensionen des Luftweges des übrigen Luftweges und die sagittale Dimension des 


\section{Diskussion}

Epipharynx unabhängig von der sagittalen und transversalen Dimension des übrigen Luftweges. Bei den Frauen zeigte sich ein ähnliches Bild.

Auf Höhe des unteren Inzisialpunktes ist bei den Männern der sagittale Luftweg unabhängig vom Epi- und Hypopharynx sowie vom Luftweg hinter der Zunge. Bei der transversalen Dimension gab es kaum Korrelationen zu den sagittalen Messwerten, dafür bis auf den Epipharynx bei jeden transversalen Messwert. Bei den Frauen zeigte sich ein zum Teil ein anderes Bild hinsichtlich der sagittalen Dimension. Dort gab es bei der sagittalen Luftweg eine Abhängigkeit $\mathrm{zu}$ allen anderen sagittalen Messwerten (außer zum Epipharynx), in der transversalen Dimension wiederrum zu allen transversalen Messwerten.

Die Luftwegsdimension auf Höhe des Isthmus verhält sich in der sagittalen Dimension nur abhängig von Isthmus transversal und anterior-posterior Ausdehnung des Luftweges auf Höh des unteren Inzisialpunktes. Dagegen ist die transversale Ausdehnung von allen Luftwegsebenen abhängig. Bei den Frauen zeigte sich ein ähnliches Bild.

Während bei den Männer der sagittale Durchmesser auf Höhe des Zunge unabhängig von fast allen anderen Dimensionen ist, ist er bei den Frauen nur von den transversalen Durchmessern unabhängig. Der transversale Durchmesser ist bei den Frauen und den Männern weitestgehend unabhängig vom sagittalen Durchmesser.

Insgesamt kann man sagen, dass die sagittalen Durchmesser zum großen Teil untereinander abhängig sind, ebenso wie die transversalen Durchmesser. Eine Abhängigkeit zwischen der sagittalen Dimension und der transversalen konnte nicht so oft festgestellt werden. Der sagittale Luftweg auf Höhe des Epipharynx hat anscheinend keine Auswirkung auf den übrigen Luftweg. Der in dieser Studie verwendeten Parameter für die Bestimmung von Zusammenhängen zwischen anatomischer Faktoren des aufsteigenden Unterkieferastes und der Luftwegsdimension war zum einen der Winkel $\alpha$ (Winkel zwischen der Innenkortikalis der Vorderkante des aufsteigenden Unterkieferastes und der Verbindungslinie der distalen Konturen des Unterkiefers) und der Winkel $\beta$ (Winkel zwischen Außenkortikalis der Vorderkante des aufsteigenden Unterkieferastes und der Verbindungslinie der distalen Konturen des Unterkiefers). Zum anderen wurden die sagittalen und transversalen Luftwegsdurchmesser auf Höhe des unteren Inzisialpunktes sowie der Isthmus axial gewählt. Dabei zeigte sich, dass bei den Männern sowohl der Winkel $\alpha$ als auch $\beta$ keinen Einfluss auf die Luftwegsdimensionen hatten. Es wäre zu erwarten gewesen, dass ein größerer Winkel $\alpha$ zu einer Vergrößerung der transversalen Dimension kommt. Dies konnte nicht beobachtet werden. Auch gab es zum sagittalen Durchmesser und zum Isthmus axial keine Zusammenhänge (Tabelle 7). Ein ähnliches Bild zeigte sich bei den Frauen. Anscheinend ist auch hier der Luftweg von den Winkeln unabhängig. Allerdings zeigte sich überraschend ein Zusammenhang zwischen dem Isthmus axial und dem Winkel $\beta$. Grund könnte ein mathematischer Zufallstreffer sein, weil es eher 


\section{Diskussion}

verwunderlich erscheint, dass eine Vergrößerung des Winkels $\beta$ zu einer Vergrößerung des Isthmus axial führt. Es wäre eher ein Einfluss des Winkel $\alpha$ auf dem transversalen Durchmesser zu erwarten gewesen. Inwiefern eine Positionsveränderung des Unterkiefers die Luftwegsdimensionen beeinflußt müssen weitere Untersuchungen klären. Dabei kann das Esmarchmanöver zur Hilfe genommen werden bei dem der Unterkiefer stark sagittal ausgerichtet wird.

Zusammenfassend konnte man feststellen, dass es zahlreiche anatomische Unterschiede in Bezug auf den oberen Luftweg zwischen den beiden Geschlechtern gibt. Diese Studie hatte einen deutlich höheren Frauenanteil (65 Frauen bei 64 Männer) als andere bisher durchgeführte Studien. Dementsprechend kann man von einer höheren Aussagekraft bei den geschlechtsspezifischen Unterschieden ausgehen. Die wichtigsten signifikanten Unterschiede waren vor allem Weichgaumenlänge, die Länge des kollapsfähigen Segments, Fläche und sagittale Ausdehnung des Luftweges auf Höhe der dorsalsten Stelle der Zunge und auf Höhe der Vallecula. Hier waren die Ergebnisse bei den Männern größer. Dagegen war der Luftweg auf Höhe des Inzisialpunktes bei den Frauen größer. Keine Unterschiede konnten im Bereich des Nasopharynx und im Bereich des Isthmus, sowie in den transversalen dimensionen festgestellt werden.

\subsection{Vergleich der Ergebnisse mit anderen Studien}

Die Hälfte der verwendeten Referenzpunkte und Messstrecken wurden aus Fernröntgenseitenbildund CT-Studien zum Vergleich herangezogen. Der Vergleich der Ergebnisse mit anderen Studien gestaltete sich schwierig, da die Untersuchung oft nur bei Männern oder im Gesamtkollektiv ohne Differenzierung zwischen den Geschlechtern erfolgte. Der Frauenanteil in diesen Studien wurde selten angegeben bzw. war nur sehr gering, sodass man die Werte der Gesamtkollektive aus anderen Studien mit den Werten der Männer aus der hier vorliegenden Studie vergleichen kann. Die andere Hälfte der Referenzpunkte variierten zu den vergleichbaren Studien und somit ebenfalls die Messstrecken (siehe 4.1.4.). Weiterhin gibt es Unterschiede in Bezug auf die Aufnahmebedingungen, die in Kapitel 4.1.2. erwähnt wurden.

\subsubsection{Messergebnisse im mediosagittalen Schnitt}

Es wurde in der mediosagittalen Ebene die Hartgaumenlänge bestimmt (Strecke ANS-PNS).

Oosterkamp et al. (2007) gaben für die Strecke ANS-PNS 54,2 mm \pm 2 und Perry et al. (2013) 60,6 $\mathrm{mm} \pm 5,7$ an. Der in der vorliegenden Studie ermittelte Wert dieser Strecke lag für die Männer bei $56,64 \mathrm{~mm} \pm 3,02$ und zeigt somit ein ähnliches Ergebnis wie die genannten Studien. Im 


\section{Diskussion}

Gesamtkollektiven wurden Werte von $48,3 \mathrm{~mm}$ bis $54,5 \mathrm{~mm}$ gemessen ( Sakakibara et al. 1999, Battagel et al. 2002 und Bae et al. 2011). Hier wird erkennbar, dass es wenige Studien gibt, die das orofaziale System nach Geschlecht (Frauen: 52,72 $\mathrm{mm} \pm 2,82$ ) unterscheiden. Für das Gesamtkollektiv gab Markiewicz (2011) einen Mittelwert von 54,74 mm \pm 3,46 an.

Für die Weichgaumenlänge gaben Muto et al. (2008) für die Frauen einen Mittelwert von 34,7 mm $\pm 3,7$ an. Das entspricht dem in dieser Studie ermittelten Mittelwert für Frauen $(35,99 \mathrm{~mm} \pm 3,61)$. Tangugsorn et al. (1995) untersuchten nur Männer und gaben einen Mittelwert von 41,23 mm \pm 4,35, Battagel et al. (2000) von 40,6 $\mathrm{mm} \pm 4,5$ und Baik et al. (2002) von 40,45 $\mathrm{mm} \pm 3,62$ an, was den gemessenen Werten dieser Studie entspricht (Männer 41,93 mm $\pm 5,24$ ). Perry et al. (2013) ermittelten mit 37,6 mm \pm 3,2 einen kleineren Wert. Dies könnte an dem geringen Patientenkollektiv von nur 10 Männern liegen.

Für die Weichgaumenbreite ermittelten Muto et al. (2008), die in ihrer Studie 31 Frauen untersuchten, einem Mittelwert von 9,7 $\mathrm{mm} \pm 6,9$. Dies entspricht der Breite, die in dieser Studie gemessen wurde $(9,31 \mathrm{~mm} \pm 2,41)$. Tangugsorn et al. (1995) und Battagel et al. (2000) ermittelten bei ihren männlichen Patienten Mittelwerte von 10,03 $\mathrm{mm} \pm 1,69$ und 10,4 $\mathrm{mm} \pm 1,3$. Dies entspricht dem in dieser Studie ermittelten Wert für Männer $(10,83 \mathrm{~mm} \pm 1,71)$. In der CT Studie von Fajdiga (2005) wurde ein Wert von $8 \mathrm{~mm} \pm 1,6$ festgestellt, welcher deutlich kleiner als der in dieser Arbeit gemessene Wert war.

Die oben beschriebene Problematik der Weichgewebsidentifikation spiegelte sich bei der Vermessung der Kontaktstrecke zwischen dem weichen Gaumen und der Zunge wider. Das zeigte sich auch bei der starken Diskrepanz der in dieser Studie ermittelten Messwerte $(21,84 \mathrm{~mm} \pm$ 17,21). Im Vergleich dazu gaben Peh et al. in ihrer Computertomographiestudie 2000 wesentlich kleinere Werte an $(11,0 \mathrm{~mm} \pm 10)$. Die Fernröntgenseitenbildstudie von Tangugsorn et al. (1995) zeigte ebenfalls eine sehr große Abweichung bei der Vermessung von der Kontaktstrecke zwischen weichem Gaumen und Zunge. Die Messergebnisse für Männer unterschieden sich um 73\% von denen der Digitalen Volumentomographie. Auch Engelke et al. (2006) wiesen deutlich kleinere Werte auf $(6,28 \mathrm{~mm} \pm 7,44)$.

Für den durchschnittlichen subpalatinalen Luftraum gaben Engelke et al. (2006) bei spontaner Zungenposition mit 12,3 $\mathrm{mm} \pm$ 5,85 an. Das ist deutlich höher als der hier ermittelte Wert 2,23 mm \pm 3,40. Die in der Studie von Engelke et al. (2006) angegebenen Wert für das Zungenrepositionsmanöver $(3.1 \mathrm{~mm} \pm 3,55)$, bei dem die Zunge aufgrund des Unterdrucks gegen den Gaumen gedrückt wird, ist mit denen in dieser Studie vergleichbar. Dementsprechend wird die Auswirkung des Schluckens auf den subpalatinalen Luftraum erkennbar, weil durch das Schlucken die Zunge an den Gaumen gedrückt wird und sich dadurch der Luftraum unter dem harten Gaumen verkleinert. Da die Aufnahmezeit 76 Sekunden betrug, konnte nicht sichergestellt werden, das sich 


\section{Diskussion}

die Zunge in der gesamten Zeit am Gaumen befand. Somit könnte es zu einer Verfälschung der Ergebnisses kommen.

Ein weiterer in der mediosagittalen Schicht ermittelter Wert war die Strecke zwischen der Epiglottisspitze und der Spina nasalis posterior. In der Studie von Johal et al. (2007) wurde für diese Strecke ein Mittelwert von 63,5 $\mathrm{mm} \pm 8,5$ angegeben. Dieser Wert war geringfügig größer als der in dieser Studie ermittelte Wert für Männer (59,52 mm × 7,83). Da in der Studie von Johal et al. (2007) auch die Messungen von 21 Frauen in das Ergebnis miteinflossen, wäre der Wert für die Männer wahrscheinlich deutlich höher. Somit wäre der Unterschied der Ergebnisse zwischen beiden Studien größer. Die Ursachen dafür könnten die oben aufgeführten Unterschiede in den Aufnahmebedingungen sein, vor allem die unterschiedliche Positionierung des Patienten während der Aufnahme. Baik et al. (2002) gaben für die Strecke zwischen einer harten Gaumenebene und Epiglottisspitze einen Mittelwert von 58,2 $\mathrm{mm} \pm$ 5,67 an. Dieser Wert entspricht sehr genau dem in der vorliegenden Studie ermittelten Wert.

Für die Strecke zwischen dem Hyoid zu der Nasallinie liegen die in dieser Studie gemessenen Mittelwerte für Männer (70,63 mm $\pm 6,71)$ geringfügig unterhalb der von Battagel und L'Estrange (1996) $(72,9 \mathrm{~mm} \pm 5,8)$ und Battagel et al. (2000) $(71,2 \mathrm{~mm} \pm 5,3)$ angegebenen Werte. Wie bei der Epiglottisspitze spielt auch hier die Positionierung des Patienten eine entscheidende Rolle. Allerdings konnten nicht alle Männer vermessen werden, weil das Aufnahmefeld des DVTs dies nicht ermöglichte, v.a. tief liegende Zungenbeine konnten nicht erfasst werden, was den Mittelwert der Ergebnisse erhöht hätte. Für Frauen liegen keine vergleichbaren Arbeiten vor mit denen man das Ergebnis vergleichen könnte $(56,97 \mathrm{~mm} \pm 5,42)$.

Für die Strecke ANS-Hyoidsenkrechte gaben Battagel und L'Estrange (1996) für Männer (64,5 mm $\pm 8,9)$ an. Diese lagen $20 \%$ höher als in der vorliegenden Studie $(53,46 \mathrm{~mm} \pm 8,40)$. Auch hier liegen die möglichen Gründen in der Positionierung und im Aufnahmefeld des DVT. Johal et al. (2007) gaben trotz des Frauenanteils im Patientenkollektiv einen wesentlich höheren Mittelwert von $61,1 \mathrm{~mm} \pm 7,5$ an. Auch für diesen Messwert gibt es leider keine vergleichbaren Studien für Frauen $(55,80 \mathrm{~mm} \pm 7,35)$.

\subsubsection{Messungen unter Bezug auf axiale Schichten}

In den axialen Ebenen wurden die Luftwegsdimensionen vermessen. Dabei wird von kranial nach kaudal vorgegangen. Für jede Ebene werden jeweils die sagittalen, transversalen und die Flächen der Luftweges bestimmt und mit anderen Studien verglichen,

Tangugsorn et al. (1995) ermittelten für den sagittalen Luftweg auf Höhe der Nasallinie (UPW anterior-posterior) für die Männer einen Mittelwert von 27,56 mm $\pm 3,0$, Solow et al. (1996) von 


\section{Diskussion}

25,69 $\mathrm{mm} \pm 2,9$ und Oosterkamp et al. (2007) von 26,2 $\mathrm{mm} \pm 4,0$, wobei sie den Luftweg auf der Verbindungslinie von Pterygomaxillaris zum Basion und nicht parallel zur Nasallinie vermaßen. Das erklärt auch den großen Unterschied zu den Ergebnissen dieser Studie (18,53 mm $\pm 2,84)$. Perry et al. (2013) kam mit 20,7 mm $\pm 2,0$ auch auf einem niedrigeren Wert. Für Frauen (18,08 mm $\pm 4,01$ ) liegen auch hier keine vergleichbaren Studien vor.

Angesichts der nur zweidimensionalen Darstellung im Fernröntgenseitenbild liegen für die transversale Dimension keine Vergleichswerte vor. Dadurch konnte auch keine Aussage über den Einfluss der Körperposition auf den transversalen Durchmesser getroffen werden. Den Mittelwert des Gesamtkollektivs für die transversale Dimension auf Höhe des harten Gaumens gab Fajdiga (2005) mit $21 \mathrm{~mm} \pm 7,0$ an. Allerdings unterscheidet er nicht nach Geschlecht. Außerdem ist das Patientenkollektiv mit 14 Patienten sehr gering. Dementsprechend ist der Vergleich schwierig. Insgesamt liegt sein Ergebnis rund 10\% unter dem in dieser Studie ermittelten Wert für Männer $(23,23 \mathrm{~mm} \pm 3,76)$.

Für die Fläche des Luftweges auf Höhe der Nasallinie gilt das Gegenteil. Da gab Fajdiga (2005) in seiner Studie einen Mittelwert von $500 \mathrm{~mm}^{2} \pm 230$ an. Dieser Wert war deutlich höher als das errechnetes Ergebnis für Männer $\left(340,10 \mathrm{~mm}^{2} \pm 84,84\right)$. Fajdiga (2005) nutze die in der CTSoftware vorhandene Möglichkeit der Flächenbestimmung. Dagegen musste in dieser Studie die Fläche auf Basis einer Formel für eine Ellipse berechnet werden, was nur einen Näherungswert ergab.

Der Durchschnittswert für die sagittale Ausdehnung auf Höhe des Inzisialpunktes (MPW) betrug bei Battagel und L'Estrange (1996) für Männer 9,9 mm \pm 3 ,1. Dieser Wert liegt etwas höher als der in dieser Studie gemessene Wert $(8,14 \mathrm{~mm} \pm 2,75)$. Das könnte daran liegen, dass die Fernröntgenseitenbilder im Sitzen aufgenommen wurden und nicht in dieser Studie in liegender Position. Für das Frauenkollektiv lagen für die sagittale Ausdehnung keine vergleichbaren Studien vor.

Für die Fläche und transversale Ausdehnung des Luftweges auf Höhe des Inzisialpunktes lagen keine vergleichbaren Studien vor.

Neben dem Luftweg wurden noch andere Strecken auf Höhe des Inzisialpunktes vermessen. Der Mittelwert $(80,14 \mathrm{~mm} \pm 4,85)$ für die intermaxilläre Raumlänge für die Männer stimmte annähernd mit dem von Battagel und L'Estrange (1996) $(80,4 \mathrm{~mm} \pm 6,4)$ und Battagel et al. (2000) (79 mm \pm 6) überein.

Die Messergebnisse der vorliegenden Fernröntgenseitenbildstudien für den geringsten sagittalen Abstand auf Höhe des Isthmus wichen deutlich von denen dieser Studie ab $(4,28 \mathrm{~mm} \pm 2,04)$. Die für die Männer angegeben Mittelwerte von Battagel und L'Estrange (1996) (9,6 mm $\pm 3,4)$; Solow et al. (1996) $(10,09 \mathrm{~mm} \pm 2,8)$ und Battagel et al. (2000) $(8,7 \mathrm{~mm} \pm 3)$ waren mehr als doppelt so 


\section{Diskussion}

groß. Gründe für diese großen Differenzen könnten die unterschiedlichen Messstrecken sein. In dieser Studie gab es durch die Verwendung einer Referenzebene eine reproduzierbare Messstrecke. In den Fernröntgenseitenbildstudien waren diese nicht vorhanden, was die Reproduzierbarkeit erschwerte. Ein weiterer Grund könnte die unterschiedliche Positionierung der Patienten während der Aufnahme sein, was zu Problemen bei dem Vergleich der Ergebnisse führte. Weitere Studien von Johal et al. (2007) $(8,4 \mathrm{~mm} \pm 3,2)$ und Sakakibara et al. (1999) (11 mm $\pm 4,4)$ zeigten für das Gesamtkollektiv ähnliche Abweichungen von den Messergebnissen für Männer. Muto et al. (2008) ermittelten für Frauen einen Mittelwert von 10,2 $\mathrm{mm} \pm 2,8$. Dieser Wert war wesentlich größer als der in dieser Studie $(4,62 \mathrm{~mm} \pm 2,05)$.

Für die transversale Dimension ermittelte Fajdiga (2005) einen Wert für das Gesamtkollektiv von $20 \mathrm{~mm} \pm 6,0$, dieser war in dieser Studie rund 20\% geringer (Männer: 17,14 mm $\pm 6,54$ ). Grund dafür könnte sein, dass es große Diskrepanzen bei der Patientengröße. In dieser Studie lagen Messergebnisse von 64 Männer vor, in der von Fajdiga (2005) nur 14.

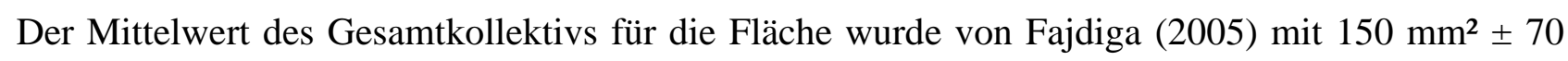
und Galvin et al. (1989) mit 134,2 $\mathrm{mm}^{2} \pm 56,6$ angegeben. Diese Mittelwerte wichen deutlich von denen in dieser Studie ab (Männer: 91,82 $\left.\mathrm{mm}^{2} \pm 57,74\right)$. Die möglichen Gründe dafür könnten die geringen Fallzahlen von Fajdiga (2005), der 14 Patienten untersuchte und Galvin et al. (1989), der 24 Patienten vermaß, sein. Abramson et al. (2009) berechneten 73,9 $\mathrm{mm}^{2} \pm 39,2$. Die gemessenen Werte lagen 25\% höher, wobei Abramson et al. (2009) nur 17 Patienten (11 Männer und 6 Frauen) untersuchten. Van Holsbeke et al. (2013) gaben dagegen 57,31 $\mathrm{mm}^{2}$ an. Die großen Unterschiede der verschiedenen Studien könnten darauf hinweisen, dass es sehr individuelle Unterschiede gibt, weil auch die Standardabweichungen sehr groß sind. Des Weiteren kann es auch an den unterschiedlichen Geräten (CT, DVT) und der Flächenberechnung liegen.

Für die sagittale Ausdehnung auf Höhe des dorsalsten Punktes der Zunge (TPW) wurde für die Männer ein Mittelwert von 11,69 mm \pm 3,99 von Tangugsorn et al. (1995), 12,4 mm \pm 3,3 von Battagel und L'Estrange (1996) und 10,8 $\mathrm{mm} \pm 3,1$ von Battagel et al. (2000) angegeben. Diese lagen im Bereich der ermittelten Werte $(11,99 \mathrm{~mm} \pm 3,56)$ dieser Studie. Muto et al. (2008) ermittelten für Frauen einen Mittelwert von 11,0 $\mathrm{mm} \pm 3,6$, welcher dem in dieser Studie angegebenen Wert von $10,14 \mathrm{~mm} \pm 2,91$ nahezu entspricht.

Für den transversalen Durchmesser gab Fajdiga (2005) einen Mittelwert von $29 \mathrm{~mm} \pm 5$ an. Die Messwerte wichen rund $15 \%$ von dem hier ermittelten Wert ab $(25,55 \mathrm{~mm} \pm 7,38)$. Im Gegensatz dazu veröffentlichten Abramson et al. (2009) leicht niedrigere Werte (22,3 mm $\pm 7,7)$. Grund für die Abweichungen in beiden Fällen könnten die wesentlich kleineren Patientenkollektive sein.

Des Weiteren gab Fajdiga (2005) für das Gesamtkollektiv für die Fläche des Luftweges einen Mittelwert von $380 \mathrm{~mm}^{2} \pm 170$ an. Dieser Wert weicht deutlich von dem dieser Studie ab (243,63 


\section{Diskussion}

$\left.\mathrm{mm}^{2} \pm 108,31\right)$. Auch hier ermittelten Abramson et al. (2009) einen kleineren Wert (147 $\mathrm{mm}^{2} \pm$ 60,1). Diese Abweichungen waren zu erwarten, da die sagittalen und transversalen Dimensionen des Luftweges bereits Unterschiede zeigten und sich damit auf die Fläche übertrugen. Auch veröffentlichten Galvin et al. (1989) und Van Holsbeke et al. (2013) für das Gesamtkollektiv einen deutlich kleineren Wert $\left(177,8 \mathrm{~mm}^{2} \pm 88,6\right.$ und 178,52 $\left.\mathrm{mm}^{2}\right)$ als der hier ermittelte Wert. Insgesamt wurden Werte der Männer mit Werten von Gesamtkollektiven verglichen in denen auch Messergebnisse der Frauen miteinflossen. Dementsprechend kann es zu dadurch zusätzlich zu Abweichungen kommen. Für Frauen $\left(197,89 \mathrm{~mm}^{2} \pm 86,54\right)$ und Männer $\left(243,63 \mathrm{~mm}^{2} \pm 108,31\right)$ lagen keine separaten Studien vor.

Für die anterior-posteriore Dimension auf Höhe der Vallecula glossoepiglottica (LPW) gaben Tangugsorn et al. (1996) für Männer einen Durchschnittswert von 20,39 mm $\pm 4,15$, Solow et al. (1996) von 18,59 $\mathrm{mm} \pm 2,27$ und Oosterkamp et al. (2007) von 14,9 mm \pm 5,2 an. Damit liegen Oosterkamp et al. (2007) im Bereich dieser Studie (Männer: 14,46 mm $\pm 2,61$ ). Die restlichen Studien weichen deutlich davon ab. Grund kann auch hier die unterschiedliche Größe des Patientenkollektivs sein. Außerdem ist die Identifizierung der Vallecula glossoepiglottica nicht einfach, sodass es dort zu Fehlern beim Vermessen kommen kann.

Der Mittelwert des Gesamtkollektivs für die Fläche des Luftweges wurde von Peh et al. (2000) mit 230,8 $\mathrm{mm}^{2} \pm 106,1$ angeben. Dier Mittelwert lag somit rund 40\% unter dem Wert meiner Studie für Männer (333,94 mm² $\pm 125,91)$. Grund könnte die Flächenberechnung sein. Peh et al. (2000) hatte per Softwareprogramm die Fläche vermessen. Des Weiteren könnten ethnische Unterschiede im Patientenkollektiv kausale Auswirkung haben. In der Studie von Peh et al. handelte es sich um Asiaten beiderlei Geschlecht, in dieser um Männer aus Europa. Außerdem weisen beide Studien eine große Schwankung der Mittelwerte auf, was erneut auf starke individuelle Unterschiede schließen lässt.

\subsubsection{Messergebnisse im koronalen Schnitt}

Im koronalen Schnitt wurde die palatinale Höhe, der Abstand der lingualen Höcker zwischen den ersten Molaren und der Abstand vom Unterkiefer zur Nasallinie bestimmt.

Für die palatinale Höhe im Bereich des ersten Molaren gaben Johal und Conaghan (2004) einen Medianwert für Männer von 18,1 mm und für Frauen von 18,75 mm an. Das Patientenkollektiv betrug jeweils 29 Patienten. Die in dieser Studie ermittelten Werte für Frauen $(22,17 \mathrm{~mm} \pm 2,63)$ und Männer $(23,26 \mathrm{~mm} \pm 3,12)$ waren deutlich größer. Dies kann an der unterschiedlichen Methodik liegen. Johal und Conaghan (2004) verwendeten Alginatabdrücke für die Ermittlung der Werte. Außerdem liegen Medianwerte vor, die den Unterschied erklären können. 


\section{Diskussion}

Auch bei dem Abstand der lingualen Höcker zwischen den ersten Molaren gab es größere Abweichungen. Für Frauen gaben Johal und Conaghan (2004) einen Wert von 45,08 mm und für Männer 46,31 mm an. Die gemessenen Werte sind damit deutlich größer als die in dieser Studie erhobenen Werte (Männer: 35,96 mm \pm 3,72, Frauen: 33,35 mm $\pm 4,04$ ).

\subsection{Einfluss der Körperposition auf den oberen Luftweg}

Es ist bekannt, dass der obere Luftweg im Wesentlichen von der Körperposition beeinflusst wird (Pae et al. 1994 und Battagel et al. 2002). Ingman et al. (2004) stellten fest, dass in Rückenlage im Vergleich zur aufrechten Position, Unterschiede im oropharyngealen Bereich auftreten können, aber nicht im naso- oder hypopharyngealen Bereich. Auch Van Holsbeke et al. (2013) zeigten in ihrer Studie, dass es morphologische Unterschiede des oberen Luftweges in aufrechter und liegender Position gibt.

Die Lagerung des Patienten hat allerdings nur Einfluss auf die Messstrecken des Weichgewebes und nicht auf die des Hartgewebes, wie z.B. die Länge des harten Gaumens. Dabei handelt es sich um knöcherne Messpunkte (Spina nasalis anterior und Spina nasalis posterior), die lageunabhängig und damit stets konstant sind. Wie von Ingman et al. (2004) beschrieben, hat die Körperposition großen Einfluss auf den Oropharynx. Dabei waren in ihrer Studie die Weichgaumenbreiten in liegender Position $(11,5 \mathrm{~mm} \pm 2,6)$ signifikant größer als in aufrechter $(10,7 \mathrm{~mm} \pm 1,8)$. Bei der Weichgaumenlänge gab es keine Unterschiede. In der vorliegenden Studie gab es weder in der Breite noch in der Länge signifikante Unterschiede zu Studien, in denen die aufrechte Position untersuchten

Diese Studie konnte Teile der Beobachtungen von Sutthiprapaporn et al. (2008) bestätigen. Sie zeigten, dass die Spitze der Epiglottis in aufrechter Position weiter kaudal und anterior liegt als in liegender Position. Eine andere Aussage von Sutthiprapaporn et al. (2008) konnte indes nicht bestätigt werden. Sie stellten fest, dass die Lage des Hyoids ebenfalls von der Körperposition abhängig war. In sitzender Position liegt das Hyoid weiter kaudal als in liegender Position. Es muss aber darauf hingewiesen werden, dass es aus technischen Gründen nicht möglich war, mit dem Digitalen Volumentomographen bei allen Patienten die Strecke zwischen dem Hyoid und der Nasallinie zu vermessen. Dementsprechend wurden sehr tiefliegende Zungenbeine nicht vermessen werden und daher wäre zu erwarten, dass die Ergebnisse für die Strecke zwischen Hyoid und Nasallinie größer sind als ermittelt. Beim Vergleich mit Fernröntgenseitenbilder konnte kein signifikanter Unterschied festgestellt werden (siehe Kapitel 4.3.1.).

Für den Luftweg auf Höhe des Nasopharynx stellten Ingman et al. (2004) fest, dass die Lagerung des Patienten keinen Einfluss auf die Luftwegsdimension hat. Mit Hilfe des Vergleichs mit 


\section{Diskussion}

Fernröntgenseitenbildern konnte die These von Ingman et al. (2004) nicht bestätigt werden. Auch Fajdiga (2005) hatte deutlich kleinere Werte als die Fernröntgenstudien. Da die Studie von Fajdiga (2005) mit einem CT durchgeführt wurde und damit auch in liegender Position, können die großen Messunterschiede im Vergleich zu den Fernröntgenseitenbildstudien ein Indiz für den Einfluss der Aufnahmeposition auf dem Nasopharynx sein. Somit kann die Rückenlage ausschlaggebend für die auftretende Verkleinerung des sagittalen Durchmessers sein. Wie sind die Unterscheide zu den Ergebnissen von Ingman et al. (2004) zu erklären? Dabei könnte die Wahl der Referenzpunkte maßgebend sein. Ingman et al. (2004) wählten einen knöchernen lageunabhängigen Punkt (Spina nasalis posterior) als anteriore und die hintere Pharynxwand als posteriore Begrenzung aus. Dadurch erfolgte die Untersuchung des Einflusses der Körperposition nur auf das posteriore Weichgewebe. Das anteriore Weichgewebe wurde dabei nicht berücksichtigt, vor allem das des weichen Gaumens.

Für den Bereich der kleinsten Stelle hinter dem weichen Gaumen und auf Uvulahöhe gaben Ingman et al. (2004) für den sagittalen Durchmesser in liegender Position $(4,2 \mathrm{~mm} \pm 3,4$ und 7,1 $\mathrm{mm} \pm 3,4)$ und in aufrechter Position an (7,2 $\mathrm{mm} \pm 2,4$ und 9,3 $\mathrm{mm} \pm 2,7)$. Die vorliegende Studie konnte das Ergebnis für den Isthmus hinter dem weichen Gaumens in liegender Position bestätigen (4,28 mm). Beim Vergleich der in dieser Studie gewonnenen Messwerte mit den von anderen Studien, welche kephalometrisch maßen, konnte ein deutlicher Unterschied in der Luftwegsdimension in aufrechter Position festgestellt werden (Kapitel 4.3.2.). Zum selben Ergebnis kamen auch Van Holsbeke et al. (2013). Sie untersuchten die Luftwegsflächen von 20 Patienten, darunter 4 Frauen, in liegender Position per CT und in aufrechter Position per DVT. Dabei stellte sich heraus, dass die Fläche hinter dem weichen Gaumen in liegender Position signifikant kleiner war als in aufrechter $\left(135,53 \mathrm{~mm}^{2} \mathrm{zu}\right.$ 230,34 $\mathrm{mm}^{2}$ ). Die Ergebnisse für die liegende Position kann die vorliegende Studie bestätigen (Männer: 123,37 mm², Frauen: 140,97 mm²).

Auch auf dem kleinsten Durchmessers des Pharynx in anterior-posteriorer Dimension hat die Körperposition einen Einfluss. Obwohl größtenteils Fernröntgenseitenbilder zum Vergleich vorlagen, konnte ein sehr großer Unterschied zwischen den Aufnahmen im Digitalen Volumentomographen und denen, die mittels Fernröntgen angefertigt wurden (Partinen et al. 1988, Tangugsorn et al. 1995, Sakakibara et al. (1999), Battagel et al. 2000, Johal et al. 2007), festgestellt werden. Es ist zu bemerken, dass das Patientenkollektiv, die Röntgentechnik und die Messpunktwahl unterschiedlich waren. Dies kann allerdings die Abweichungen von teils über 100\% nicht erklären. Dies zeigt sich auch bei der Fläche des Isthmus. Van Holsbeke et al. (2013) gaben für die liegende 57,31 $\mathrm{mm}^{2}$ und für die aufrechte Position 78,70 $\mathrm{mm}^{2}$ an. Das zeigt deutlich, dass vor allem im Bereich des weichen Gaumens die Körperposition eine entscheidene Rolle in Hinblick auf die oberen Luftwegsflächen spielt. Die vorliegende Studie ermittelte deutlich höhere Werte für 


\section{Diskussion}

die Isthmusfläche (Männer: 91,82 mm ², Frauen: 94,89 mm²). Gründe dafür könnten sein, dass diese Studie für die Flächenberechnung die Ellipsenformel verwendete, weil mit dem NewTom QR 9000 keine Flächenberechnung möglich ist. Des weiteren könnte es durch die Messung parallel zu der Nasallinie zu einer Vergrößerung der Isthmusdimensionen kommen.

Der Einfluss auf den oberen Luftwegverringert sich offensichtlich unterhalb des Velums. Die Werte für den anterior-posterioren Durchmessers hinter der Zunge waren in den Fernröntgenstudien und der vorliegenden Studie annähernd gleich (etwa $12 \mathrm{~mm}$ ). Gleiches stellte Van Holsbeke et al. (2013) für die Fläche hinter der Zunge fest. In ihrer Studie ermittelten sie keinen signifikanten Unterschied für diese Fläche (liegend: 178,52 mm², aufrecht: 195,88 m²). Man hätte erwartet, dass sich durch die liegende Position die Zunge nach dorsal verlagert und damit sich der Luftweg deutlicher verkleinert. Weiterhin gaben sie an, dass auch im Hypopharynx die Körperposition eine untergeordnete Rolle auf die Luftwegsfläche spielt. Auch dort gab es keinen signifikanten Unterschied. zwischen den einzelnen Positionen.

Insgesamt kann man feststellen, dass die größten Luftwegsveränderungen sich hinter dem weichen Gaumen befinden (Van Holsbeke et al. 2013).

Inwiefern der transversale Durchmesser von der Positionierung des Patienten abhängig ist, konnte in dieser Studie leider nicht geklärt werden. Dazu liegen keine Vergleichswerte in aufrechter Position vor.

\subsection{Schlussfolgerung}

Durch die zunehmende Nutzung der dreidimensionalen Diagnostik in der zahnärztlichen Praxis wird die Verbreitung des DVTs zunehmen.

Diese Studie hat gezeigt, dass ohne weitere Strahlenbelastung, der Luftweg und angrenzende Strukturen dargestellt und vermessen werden können. Insgesamt kann mit dem DVT ein Informationsgewinn an anatomischen Parametern erzielt werden, der für die Diagnostik eine entscheidende Rolle sein kann. Das sind vor allem die transversalen Dimensionen des Luftweges und die Flächenbestimmung des oberen Luftweges.

Insgesamt konnte mit der Studie zahlreiche Gemeinsamkeiten zwischen den Geschlechtern festgestellt werden. Diese zeigten sich beim subpalatinalen Luftraum, beim Luftweg auf Höhe der Nasallinie, beim Luftweg auf Höhe des Isthmus und die transversalen Luftwegsdimensionen auf Höhe des Inzisialpunktes, des dorsalsten Punktes der Zunge und der Vallecula glossoepiglottica. Unterschiede wurden vor allen in den mediosagittalen Schicht festgestellt. Im Einzelnen waren dies: die Hartgaumenlänge, die Weichgaumenlänge und-breite, die Strecke PNS-Epiglottisspitze, die Strecke Hyoid-Nasallinie. Ferner waren Unterschiede bei den sagittalen Luftwegsdimensionenen 
und Luftwegsquerschnitte auf Höhe des Inzialpunktes, des dorsalsten Punktes der Zunge und der Vallecula glossoepiglottica zu beobachten.

Die Körperposition hat offensichtlich nur im Bereich hinter dem Velum einen Einfluss auf die Luftwegsdimension. D.h. Obstruktionen sind im Bereich hinter dem weichen Gaumen zu erwarten. Zur Qualitätsverbesserung und Standardisierung der Aufnahmen ist es sinnvoll, das Zungenrepositionsmanöver unter Kontrolle einer Membrantrichterplatte durchzuführen und die Interkuspitation sicherzustellen, um dadurch eine Stabilisierung der Zunge in der Mundhöhle zu gewährleisten.

Des Weiteren könnte durch eine verringerte Aufnahmezeit die Qualität der DVT-Datensätze verbessert werden, weil damit die Bewegungsartefakte und -unschärfen reduziert werden könnten. 


\section{Zusammenfassung}

Fragestellung. Mit der Digitalen Volumentomographie (DVT) wurde 1997 in die Zahn-, Mundund Kieferheilkunde eine neue Aufnahmetechnik eingeführt, die dieselben Rekonstruktionsmöglichkeiten wie die Computertomographie (CT) und Magnetresonanztomographie (MRT) bietet. Auf der Basis vorhandener DVT-Datensätze sollte der Luftweg im Bereich des orofazialen Systems vermessen werden. Die dabei gemessenen Parameter dienten der Erstellung von Normwerten in horizontaler Körperposition mit Schwerpunkt der Ermittlung geschlechtsspezifischer Unterschiede. Des Weiteren sollten die Zusammenhänge anatomischer Faktoren des aufsteigenden Unterkieferastes und der Luftwegsdimension dargestellt werden.

Methodik. Die Bestimmung des oberen Luftweges wurde an 129 Patienten (65 Frauen und 64 Männer), im Alter zwischen 30 und 68 Jahren, vorgenommen, die aufgrund anderer Indikationsstellung mittels des Digitalen Volumentomographen NewTom QR-DVT 9000@ mit einer Röhrenspannung von $110 \mathrm{kV}$ und einem Röhrenstrom zwischen 0,5-10 mA geröntgt wurden. Dabei mussten die anatomischen Strukturen von der Nasallinie bis zum Hyoid abgebildet sein. Die Auswertung wurde mit dem Softwareprogramm NewTom 9000 Dental Version 3.37 durchgeführt. Im Rahmen der Untersuchung wurden unteranderen folgende Parameter bestimmt: die Hartgaumenlänge, die Weichgaumenlänge und Weichgaumenbreite, die Länge des kollapsfähigen Segments, der Abstand von Hyoid zur Nasallinie, die sagittalen Dimension auf Höhe des Inzisialpunktes (MPW), auf Höhe des dorsalsten Punktes der Zunge (TPW) und auf Höhe der Vallecula glossoepiglottica (LPW), sowie die Neigung des Unterkieferastes zur mediosagittalen Ebene (Winkel $\alpha$ und Winkel $\beta$ ). Es wurde eine deskriptive Auswertung durchgeführt. Der Vergleich der metrischen Werte erfolgte mit Hilfe von Korrelationsmatrizen nach Pearson. Die Gegenüberstellung zweier unabhängiger Stichproben (Vergleich Geschlecht mit metrischen Werten) wurde mit dem Mann-Whitney-U-Test durchgeführt. Das Signifikanzniveau wurde auf $\mathrm{p}<0,05$ festgelegt.

Resultate: Bei Messungen in der mediosagittalen Ebene wurden signifikante Unterschiede zwischen den Geschlechtern festgestellt: bei der Hartgaumenlänge (Männer: 56,64 mm \pm 3,02, Frauen: 52,72 $\mathrm{mm} \pm 2,82$ ), bei der Weichgaumenlänge (Männer: 41,93 $\mathrm{mm} \pm 5,24$, Frauen: 35,99 $\mathrm{mm} \pm 3,61$ ) und Weichgaumenbreite (Männer: 10,83 $\mathrm{mm} \pm 1,71$, Frauen: 9,31 $\mathrm{mm} \pm 2,41$ ), die Länge des kollapsfähigen Segments (Männer: 59,52 mm \pm 7,83, Frauen: 50,38 mm \pm 5,27) und beim Abstand von Hyoid zur Nasallinie (Männer: 70,63 mm \pm 6,71, Frauen: 56,97 mm \pm 5,42). Auch bei den Luftwegsdimensionen gab es signifikante Unterschiede und zwar in der sagittalen Dimension auf Höhe des Inzisialpunktes (MPW) (Männer: 8,14 mm $\pm 2,75$, Frauen: 9,24 mm \pm 
3,15), auf Höhe des dorsalsten Punktes der Zunge (TPW) (Männer: 11,99 mm \pm 3,56, Frauen: 10,14 mm $\pm 2,91$ ) und auf Höhe der Vallecula glossoepiglottica(LPW) (Männer: 14,46 mm \pm 2,61, Frauen: 11,55 mm $\pm 2,50$ ). Auch bei den Querschnittsflächen konnte signifikante Unterschiede festgestellt werden: auf Höhe des Inzisialpunktes (MPW) (Männer: 123,37 mm² $\pm 82,52$, Frauen: 140,97 $\mathrm{mm}^{2} \pm 64,98$ ), auf Höhe des dorsalsten Punktes der Zunge (TPW) (Männer: 243,63 mm² \pm 108,31, Frauen: 197,89 mm² \pm 86,54) und auf Höhe der Vallecula glossoepiglottica(LPW) (Männer: $333,94 \mathrm{~mm}^{2} \pm 125,91$, Frauen: 276,06 $\left.\mathrm{mm}^{2} \pm 94,56\right)$. Keine signifikante Unterschiede konnte in den transversalen Durchmessern, beim Luftquerschnitt auf Höhe des Isthmus (Männer: 91,82 mm² \pm 57,74, Frauen: $94,89 \mathrm{~mm}^{2} \pm 51,98$ ) und bei der Neigung des Unterkieferastes zur mediosagittalen Ebene (Winkel $\alpha$ und Winkel $\beta$ ) festgestellt werden.

Schlussfolgerung. Insgesamt konnte man feststellen, dass es zahlreiche anatomische Unterschiede in Bezug auf den oberen Luftweg zwischen den beiden Geschlechtern gibt. Der hohe Frauenanteil ermöglichte eine höhere Aussagekraft in Bezug auf den Geschlechtervergleich. Die wichtigsten signifikanten Unterschiede waren vor allem Weichgaumenlänge und- breite, die Länge des kollapsfähigen Segments, der Abstand von Hyoid und Nasallinie, Querschnitte und sagittale Ausdehnung des Luftweges auf Höhe der dorsalsten Stelle der Zunge und auf Höhe der Vallecula glossoepiglottica. Hier waren die Ergebnisse bei den Männern deutlich größer. Dagegen war der Luftweg auf Höhe des Inzisialpunktes bei den Frauen größer. Keine Unterschiede konnten im Bereich des Nasopharynx und im Bereich des Isthmus, sowie in den transversalen Dimensionen festgestellt werden.

Auch die Rolle der Körperposition muss bei der Auswertung miteinbezogen werden. Anscheinend hat die Körperposition nur im Bereich hinter dem Velum einen Einfluss auf die Luftwegsdimension. In liegender Position scheinen die Luftwegsquerschnitte deutlich kleiner zu sein als in aufrechter.

Das NewTom QR-DVT 9000@ liefert wertvolle Informationen über die Luftwegsdimensionen in horizontaler Lage. 


\section{Abkürzungsverzeichnis}

Abb.: Abbildung

ANS: Spina nasalis anterior

BV: Bildverstärker

$\mathrm{cm}$ : Zentimeter

CT: der Computertomograph, die Computertomographie, das Computertomogramm

3D: dreidimensional

DVT: der Digitale Volumentomograph, die Digitale Volumentomographie, das Digitale

Volumentomogramm

Korr.: Korrelation

kV: Kilovolt

LPW: Lower Pharyngeal Wall, Luftweg auf Höhe der Vallecula glossoepiglottica

M.: Musculus

$\mathrm{mA}$ : Milliampere

mAs: Milliamperesekunde

Max.: Maximum

mGy: Milligray

Min.: Minimum

Mm.: Musculi

mm: Millimeter

MPW: Middle Pharyngeal Wall, Luftweg auf Höhe des unteren Inzisialpunktes

MRT: der Magnetresonanztomograph, die Magnetresonanztomographie, das

Magnetresonanztomogramm

$\mu$ Sv: Mikrosievert

mSv: Millisievert

N.: Nervus

NL: Nasallinie

Nn.: Nervi

OSAS: Obstruktives Schlafapnoesyndrom

PNS: Spina nasalis posterior

S.: Seite

Stdabw:: Standardabweichung

Tab.: Tabelle 
TPW: Tongue Pharyngeal Wall, Luftweg auf Höhe der dorsalsten Stelle der Zunge UK: Unterkiefer

UPW: Upper Pharyngeal Wall, Luftweg auf Höhe der Nasallinie

Var.: Variablen 


\section{Anlagen}

\subsection{Fallbeispiele}

\subsubsection{Schnitt in der mediosagittalen Ebene}
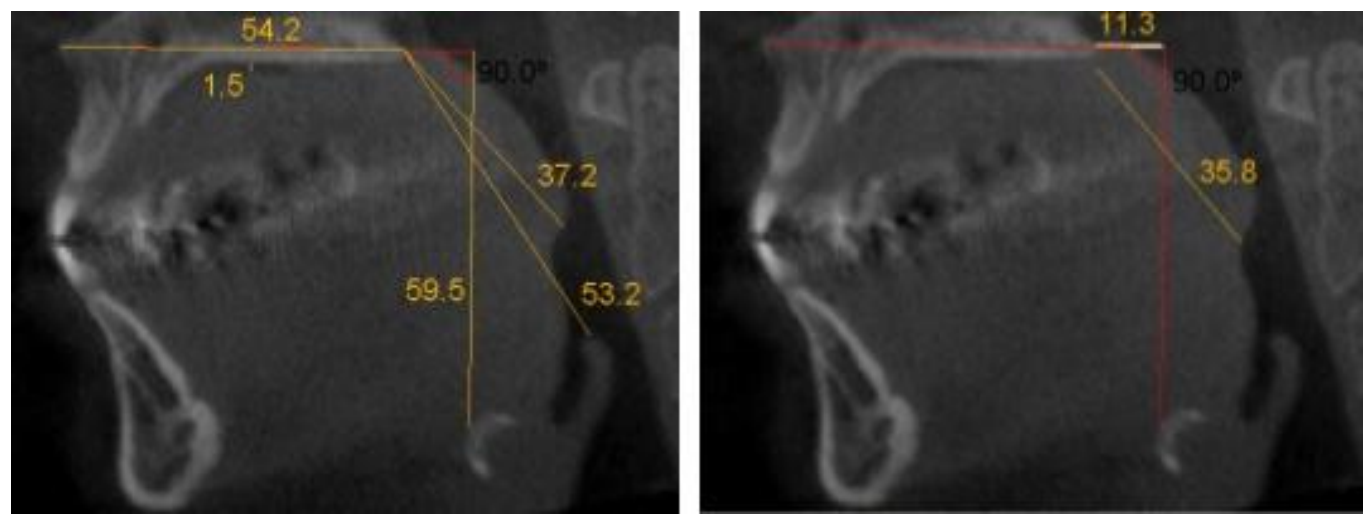

Abb. 44: Mediosagittale Schnitte

Die DVT-Aufnahmen zeigen den durchgeführten mediosagittalen Schnitt (Abb. 52) und die durchgeführten Messstrecken (Strecke ANS-PNS, Weichgaumenlänge, Strecke PNSEpiglottisspitze, Strecke Hyoid zur Nasallinie, Strecke der Hyoidsenkrechten zur ANS, der subpalatinale Luftraum und die Kontaktstrecke zwischen der Zunge und dem weichen Gaumen). Es ist deutlich zu erkennen, dass eine genaue Abgrenzung zwischen weichem Gaumen und der Zunge nicht möglich ist.

\subsubsection{Axialer Schnitt auf Höhe des unteren Inzisialpunktes (MPW)}

Die DVT-Aufnahmen (Abb. 53) zeigen den durchgeführten axialen Schnitt auf Höhe des unteren Inzisialpunktes (MPW) und die durchgeführten Messstrecken (intermaxilläre Raumlänge, MPW anterior-posterior, MPW transversal) sowie die gemessenen Winkel $\alpha$ und $\beta$. Hier ist auch deutlich die Artefaktbildung bedingt durch die prothetische Versorgung erkennbar.
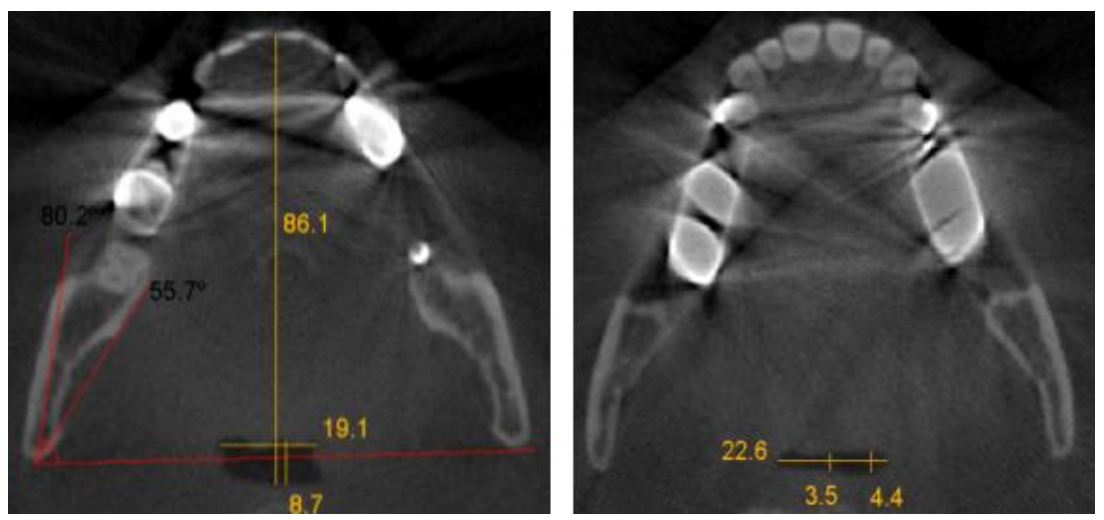

Abb. 45: Schnitt auf Höhe der Inzisialkante 


\subsubsection{Koronaler Schnitt auf der Hälfte der Nasallinie}

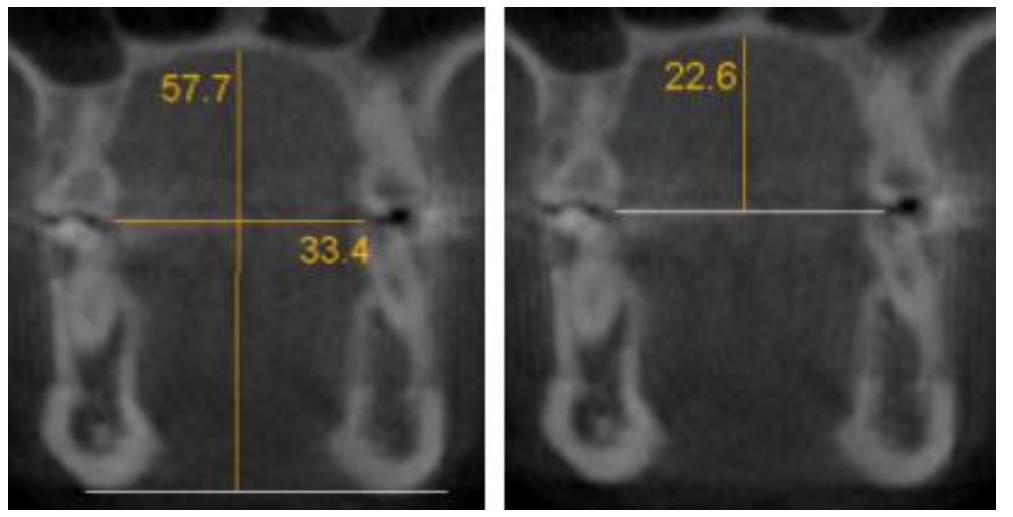

Abb. 46: Koronaler Schnitt auf der Hälfte der Nasallinie

Die DVT-Aufnahmen zeigen den durchgeführten koronalen Schnitt auf Höhe der Hälfte der Nasallinie und die durchgeführten Messstrecken (Strecke Nasallinie zu Unterkiefer, palatinale Höhe, Abstand linguale Höcker) (Abb. 54). 


\section{Literaturverzeichnis}

\section{Literaturverzeichnis}

Abramson Z, Susarla S, Troulis M, Kaban L (2009): Age-related changes of the upper airway assessed by 3-dimensional computed tomography. J Craniofac Surg 20(5): 1629-1630

Anderson DM: Dorland’s Illustrated Medical Dictonary 29th Edition. W.B. Saunders Company Philadelphia 2000

Ash MM, Nelson SJ (2003): Wheeler's Dental Anatomy, Physiology and Occlusion 8th edition. Saunders Philadelphia 417-419

Arai Y, Tammisalo E, Hashimoto K, Shinoda K (1999): Development of a compact computed tomographic apparatus for dental use. Dentomaxillofac Radiol 28: 245-248

Avrahami E, Englender M (1995): Relation between CT Axial Cross-sectional Area of the Oropharynx and Obstructive sleep Apnea Syndrome in Adults. Am J Neuroradiol 16: 135-140

Avrahami E, Solomonovich A, Englender M (1996): Axial CT Measurements of the Cross-sectional Area of the Oropharynx in Adults with Obstructive sleep Apnea Syndrome. Am J Neuroradiol 17: $1107-1111$

Bae Y, Kuehn DP, Sutton BP, Conway CA, Perry JL (2011): Three dimensional magnetic resonance imaging of velopharyngeal structures. J Speech Hear Res 54: 1538-1545

Baik UB, Suzuki M, Ikeda K, Sugawara J, Mitani H (2002): Relationship Between Cephalometric Characteristics and Obstructive Sites in Obstructive Sleep Apnea Syndrome. Angle Orthod 72: 124134

Battagel JM, L'Estrange PR (1996): The cephalometric morphology of patients with obstructive sleep apnoea (OSA). Eur J Orthod 18: 557-569

Battagel JM, Johal A, Kotecha B (2000): A cephalometric comparison of subjects with snoring and obstructive sleep apnoea. Eur J Orthod 22: 353-365 


\section{Literaturverzeichnis}

Battagel JM, Johal A, Smith AM, Kotecha B (2002): Postural variation in oropharyngeal dimensions in subject with sleep disordered breathing: a cephalometric study. Eur J Orthod 24: 263276

Becker M (1998): Larynx and hypopharynx. Radiol Clin North Am 무: 891-920

Bohlman ME, Haponik EF, Smith PL, Allen RP, Bleecker ER, Goldman SM (1983): CT Demonstration of Pharyngeal Narrowing in Adult Obstructive Sleep Apnea. AJR 140: 543-548

Brugmans MJ, Buijs WC, Geleijns J, Lembrechts J (2002): Population exposure to diagnostic use of

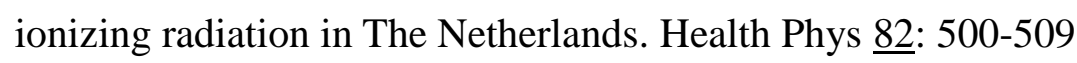

Cacaci C, Frank E, Bumann A (2007): DVT-Volumentomograph Digitaler Durchblick. J Cont Dent Educ 3: 1-9

Cohnen M, Kemper J, Möbes O, Pawelzik J, Mödder U (2002): Radiation dose in dental radiology. Eur Radiol 12: 634-637

Curtis DJ (1986): Radiographic anatomy of the pharynx. Dysphagia 1: 51-62

Czerny C, Formanek M (2000): Malignant tumors of the pharynx. Radiologe 40: 625-631

Danforth RA (2003): Cone beam volume tomography: a new digital imaging option for dentistry. J Calif Dent Assoc 31: 814-815

Dirsch P (2008): Digitale Volumentomografie in der zahnärztlichen Implantologie. ZWP spezial 7+8: $10-14$

Drenckhahn D, Zenker W: Benninghoff Anatomie 15. Auflage Band 1. Urban \& Schwarzenberg München 2002

Drescher D: Fernrontgenanalyse: Praxis der Zahnheilkunde. Kieferorthopadie I. Band. 11/1. 4. Auflage. Urban \& Fischer München 2000 


\section{Literaturverzeichnis}

Engelke W (2007): Systematische Rhonchopathiebehandlung in der zahnärztlichen Praxis. DFZ 7 ㅇ: $49-51$

Engelke W, Mendoza M, Repetto G (2006): Preliminary radiographic observations of the tonguerepositioning Manoeuvre. Eur J Orthod 28: 618-623

Ekberg O (1989): Die radiologische Beurteilung des Pharynx. Radiologe 29: 285-294

Fairburn SC, Waite PD, Vilos G, Harding SM, Bernreuter W, Cure J, Cherala S (2007): ThreeDimensional Changes in Upper Airways of Patients With Obstructive Sleep Apnea Following Maxillomandibular Advancement. J Oral Maxillofac Surg 65: 6-12

Fajdiga I (2005): Snoring Imaging Could Bernoulli Explain It All? Chest 128: 896-901

Frank E, Frank S (2007): Augen auf beim DVT-Kauf. Z Zahnärztl Implantol 23: 313-315

Freisfeld M (1973): Fehlerquellen an Einzeichnungsserien kephalometrischer Bezugspunkte. Fortschr Kieferorthop 34: 296-306

Fuhrmann A, Schulze D, Rother U (2003): Digital transversal slicebimaging in dental-maxillofacial radiology: from pantomography to digital volume tomography. Int J Comput Dent $\underline{6}$ : 129-140

Galanski M, Nagel HD, Stamm G (2001): CT-Expositionspraxis in Deutschland. Fortschr Röntgenstr 173: 1-66

Galvin JR, Rooholamini SA, Stanford W (1989): Obstructive Sleep Apnea: Diagnosis with Ultrafast CT. Radiology 171: 775-778

Goch I: Vergleich metrischer Genauigkeit von Computertomographie, Digitaler Volumentomographie und Orthopantomographie in der modernen Implantologie. Med. Diss. Düsseldorf 2005

Hanazawa T, Sano T, Seki K, Okano T (2004): Radiologic measurements of the mandible: a comparison between CT-reformatted and conventional tomographic images. Clin Oral Implants Res 15(2): 226-232 


\section{Literaturverzeichnis}

Hirsch E (2002): DVT Digitale Volumentomographie zur präoperativen Darstellung verlagerter Zähne. ZMK 18(6): 394

Hochban W, Brandenburg U, Schürmann R (1994): Zur Gesichtsskelettmorphologie beim obstruktiven Schlafapnoe-Syndrom und ihrem Einfluß auf die Atemwegsobstruktion. DZZ 49: 777782

Holberg C, Steinhäuser S, Geis P, Rudzki-Janson I (2005): Cone-Beam Computed Tomography in Orthodontics: Benefits and Limitations. Die Digitale Volumentomographie in der Kieferorthopädie: Möglichkeiten und Grenzen. J Orofac Orthop 66: 434-444

Hora F, Nápolis LM, Daltro C, Kodaira SK, Tufik S, Togeiro SM, Nery LE (2007): Clinical, Anthropometric and Upper Airway Anatomic Characteristics of Obese Patients with Obstructive Sleep Apnea Syndrome. Respiration 74: 517-524

Horner RL, Mohiaddin RH, Lowell DG, Shea SA, Burman ED, Longmore DB, Guz A (1989): Sites and sizes of fat deposits around the pharynx in obese patients with obstructive sleep apnoea and weight matched controls. Eur Respir J 2(7): 613-622

Houston WJB (1983): The analysis of errors in orthodontic measurements. Am J Orthod 83: $382-390$

Hübinger A: Evaluation dreidimensionaler radiologischer Verfahren zur Darstellung der Mandibula am Humanpräparat. Med. Diss. Gießen 2008

Hupfauf L (1971): Vergleichende Untersuchung verschiedener Registrierverfahren. DZZ 26: 158162

Ingman T, Nieminen T, Hurmerinta K (2004): Cephalometric comparison of pharyngeal changes in subjects with upper airway resistance syndrome or obstructive sleep apnoea in upright and supine positions. Eur J Orthod 26: 321-326

Jeong DK, Lee SC, Huh KH, Yi WJ, Heo MS, Lee SS, Choi SC (2012): Comparison of effective dose for imaging of mandible between multi-detector CT and cone-beam CT. Imaging Sci Dent 42(2): $65-70$ 


\section{Literaturverzeichnis}

Johal A, Conaghan C (2004): Maxillary morphology in obstructive sleep apnea: a cephalometric an model study. Angle Orthod 74: 648-656

Johal A, Patel SI, Battagel JM (2007): The relationship between craniofacial anatomy and obstructive sleep apnoea: a case-controlled study. J Sleep Res 16: 319-326

Kaul A, Bauer B, Bernhardt J, Nosske D, Veit R (1997): Effective doses of members of the public from the diagnostic application of ionizing radiation in Germany. Eur Radiol 7:1127-1132

Koeck B, Bierwirth JT (1986): Die Veränderung der Ruheschwebe des Unterkiefers in Abhängigkeit von Schwerkraft, Kopf- und Körperhaltung. DZZ 41: 1161

Koren A, Gronelj LD, Fajdiga I (2009): CT comparison of primary snoring and obstructive sleep apnea syndrome: role of pharyngeal narrowing ratio and soft palate-tongue contact in awake patient. Eur Arch Otorhinolaryngol 266 (5): 727-734

Latta Jr GH (1992): Influence of circadian periodicity on reproducibility of centric relation records for edentulous patients. J Prosthet Dent $\underline{68}$ : 780-783

Lippert H: Lehrbuch Anatomie 7. Auflage. Urban \& Fischer Verlag München/Jena 2006

Lomoschitz F, Schima W, Schober E, Pokieser P, Youssefzadeh S, Kainberger F, Czerny C, Imhof H (2000): The pharynx. The imaging of its normal anatomy. Radiologe 40(7): 601-609

Lotzmann U: Die Prinzipien der Okklusion 5. Auflage. Neuer Merkur München 1998

Lowe A, Takada K, Yamagata Y, Econ B, Sakuda M (1985): Dentoskeletal and tongue soft-tissue correlates: A cephalometric analysis of rest postion. Am J Orthod 88: 333-341

Lowe A, Santamaria JD, Fleetham JA, Price C (1986): Facial morphology and obstructive sleep apnea. Am J Orthod Dentofac Orthop 90: 484-491

Lüllmann-Rauch R: Taschenlehrbuch Histologie 2. Auflage. Georg Thieme Verlag Stuttgart 2006 


\section{Literaturverzeichnis}

Markiewicz D: Beurteilung des oberen Luftweges bei Patienten mit schlafbezogenen Atemstörungen im Digitalen Volumentomographen (DVT). Med. Diss. Göttingen 2011

Maurer JT, Stuck BA (2008): Update: Diagnostik der oberen Atemwege bei Schlafapnoe-Syndrom. HNO $\underline{56}: 1089-1097$

Moebes O, Becker J, Schnelle C, Ewen K, Kemper J, Cohnen M (2000): Strahlenexposition bei der digitalen Volumentomographie, Panoramaschichtaufnahme und Computertomographie. DZZ 55: $335-339$

Moll KJ, Moll M: Anatomie 18. Auflage. Urban \& Fischer Verlag München/Jena 2006

Moss ML (1983): Beyond roentgenographic cephalometry - What? Am J Orthod 또: 77-79

Moyer RE, Bookstein FL (1979): The inappropiateness of conventional cephalometrics. Am J Orthod 75: 599-617

Mozzo P, Procacci C, Tacconi A, Tinazzi Martini P, Bergamo Amdreis JA (1998): A new volumetric CT machine for dental imaging based on the cone beam technique: preliminary results. Eur Radiol $\underline{8}: 1558$

Muto T, Yamazaki A, Takeda S (2008): A cephalometric evaluation of the pharyngeal airway space in patients with mandibular retrognathia and prognathia, and normal subjects. Int J Oral Maxillofac Surg 37: 228-231

Novelline RA : Squire's Radiology. Grundlagen der klinische Diagnostik für Studium und Praxis. Schattauer Verlagsgesellschaft mbH Stuttgart 2001

Obrez A, Stohler CS (1996): Jaw muscle pain and its effect on gothic arch tracings. J Prosthet Dent 75: 393-398

Obrez A, Türp JC (1998): The effect of musculoskeletal facial pain on registration of maxillomandibular relationships and treatment planning: a synthesis of the literature. J Prosthet Dent 79: 439-445 


\section{Literaturverzeichnis}

Oettinger B, Oettinger T: Anatomie 9. Auflage. BON MED Verlag GmbH Lorch 1992

Oosterkamp BCM, Remmelink HJ, Pruim GJ, Hoekema A, Dijkstra PU (2007): Craniofacial, Craniocervical, and Pharyngeal Morphology in Bilateral Cleft Lip and Palate and Obstructive Sleep Apnea Patients. Cleft Palate-Craniofac J 44 (1): 1-7

Pae EK, Lowe AA, Sasaki K, Price C, Tsuchiya M, Fleetham JA (1994): A cephalometric and electromyographic study of upper airway structures in the upright and supine position. Am J Orthod Dentofac Orthop 106: 52-59

Partinen M, Guilleminault C, Quera-Salva MA, Jamieson A (1988): Obstructive Sleep Apnea and Cephalometric Roentgenograms. The role of anatomic upper airway abnormalities in the definition of abnormal breathing during sleep. Chest 93 (6): 1199-1205

Pasler FA: Zahnärztliche Radiologie. 5. Auflage. Georg Thieme Verlag Stuttgart 2008

Peh WCG, Ip MSM, Chu FSK, Chung KF (2000): Computed tomographic cephalometric analysis of Chinese patients with obstructive sleep apnoea. Australas Radiol 44: 417-423

Pepin JLD, Veale D, Ferretti GR, Mayer P, Levy PA (1999): Obstructive Sleep Apnea Syndrome: Hooked Appearance of the Soft Palate in Awake Patients-Cephalometric and CT Findings. Radiology 210: 163-170

Perry JL, Sutton BP, Kuehn DP, Gamage JK (2013): Using MRI for Assessing Velopharyngeal Structures and Function. Ckeft Palate Craniofac J 50 (6)

Pickuth D, Frommhold H, Müller-Gärtner HW: Klinische Radiologie systematisch. Diagnostische Radiologie, Nuklearmedizin, Strahlentherapie in 2 Bänden, 1. Band Uni-Med Verlag, Bremen 1998

Pracharktam N, Nelson S, Mark GH, Broadbent BH, Redline S, Rosenberg C, Strohl KP (1996): Cephalometric assessment in obstructive sleep apnea. Am J Orthod Dentofac Orthop 109: 410-419

Pradel W, Schmidt F, Paditz E, Eckelt U (2000): Stellenwert der Radiokephalometrie in der Diagnostik bei OSAS im Erwachsenalter. Somnologie 4: 96-100 


\section{Literaturverzeichnis}

Probst R, Grevers G, Iro H: Hals- Nasen- Ohren- Heilkunde 2. Auflage. Georg Thieme Verlag Stuttgart 2004

Prokop M (2002): Überblick über die Strahlenexposition und Bildqualität in der Computertomographie. Fortschr Röntgenstr 174: 631-636

Pschyrembel: Klinisches Wörterbuch. 262. Auflage. de Gruyter Berlin New York 2011

Reich RH (1980): Anatomische Untersuchungen zum Verlauf des Canalis mandibularis. DZZ $\underline{35}$ : 972-975

Rother UJ: Moderne bildgebende Diagnostik in der Zahn-, Mund- und Kieferheilkunde. 2. Auflage. Elsevier GmbH München 2006

Sakakibara H, Tong M, Matsushita K, Hirata M, Konishi Y, Suetsugu S (1999): Cephalometric abnormalities in non-obese and obese patients with obstructive sleep apnoea. Eur Respir J 13: 403410

Scarfe WC, Farman AG, Sukovic P (2006): Clinical applications of conebeam computed tomography in dental practice. J Can Dent Assoc $\underline{72(1): 75-80}$

Scherer P, Neugebauer J, Ritter L, Mischkowski R, Scheer M, Zöller JE (2007): Indikationen für die 3-dimensionale Bildgebung in der Zahnheilkunde. ZWR 116: 219-230

Schiebler TH, Korf HW: Anatomie 10. Auflage. Steinkopff Verlag Darmstadt 2007

Schnelle C: Vergleich der Strahlenexposition bei der Digitalen-Volumen-Tomographie, der Panoramaschichtaufnahme und der Computertomographie. Med. Diss. Düsseldorf 2001

Schopf P: Curriculum Kieferorthopädie Band I. 2. Aufl. Quintessenz Berlin, London, Sao-Paulo 1994: 185-187

Schopf, P: Curriculum Kieferorthopädie Band I und II 4. Auflage. Quintessenz Berlin, London, Sao Paulo 2008 


\section{Literaturverzeichnis}

Schug-Kösters M (1968): Röntgenologisch-anatomische Studien am Canalis mandibularis und dessen Abzweigungen unter Berücksichtigung differential-diagnostischer Besonderheiten. DZZ 7: $331-338$

Schwenzer N, Ehrenfeld M: Allgemeine Chirurgie Band 1. 3. Auflage. Georg Thieme Verlag Stuttgart 2000

Segner, D, Hasund A: Individualisierte Kephalometrie. Hansa Hamburg 1998

Shafagh I, Yoder JL, Thayer KE (1975): Diurnal variance of centric relation position. J Prosthet Dent 34: 574-582

Solow B, Skov S, Ovesen J, Norup PW, Wildschiodtz G (1996): Airway dimensions and head posture in obstructive sleep apnoea. Eur J Orthod 18: 571-579

Sömmer C: Radiologische Querschnittsstudie zur Qualitätsverbesserung von Panoramaschichtaufnahmen mittels Zungenrepositionsmanöver Med. Diss. Göttingen 2008

Strocchi S, Colli V, Conte L (2012): Multidetector CT fluoroscopy and cone-beam CT-guided percutaneous transthoracic biopsy: comparison based on patient doses. Radiat Prot Dosimetry 151(1):162-5

Suomalainen A, Vehmas T, Kortesniemi M, Robinson S, Peltola J (2008): Accuracy of linear measurements using dental cone beam and conventional multislice computed tomography. Dentomaxillofac Radiol 37(1): 10-17

Sutthiprapaporn P, Tanimoto K, Ohtsuka M, Nagasaki T, Iida Y, Katsumata A (2008): Positional changes of oropharyngeal structures due to gravity in the upright and supine positions. Dentomaxillofac Radiol 37 (3): 130-135

Tangugsorn V, Skatvedt O, Krogstad O, Lyberg T (1995): Obstructive sleep apnoea: a cephalometric study. Part II. Uvulo-glossopharyngeal morphology. Eur J Orthod 17: 57-67 


\section{Literaturverzeichnis}

Teitelbaum J, Diminutto M, Comiti S, Pépin JL, Deschaux C, Raphaël B, Bettega G (2007): Lateral cephalometric radiography of the upper airways for evaluation of surgical treatment of obstructive sleep apnea syndrome. Rev Stomatol Chir Maxillofac 108 (1): 13-20

Thiel HJ, Hassfeld S: Cone-Beam CT: Digitale Volumentomographie für den Zahn- und Kieferbereich, in Schnittbilddiagnostik in der MKG Medizin und Zahnmedizin. Georg Thieme Verlag Stuttgart 2001

Valiyaparambil JV, Yamany I, Ortiz D, Shafer DM, Pendrys D, Freilich M, Mallya SM (2012):

Bone quality evaluation: comparison of cone beam computed tomography and subjective surgical assessment. Int J Oral Maxillofac Implants 27(5): 1271-7

Van Holsbeke CS, Verhulst SL, Vos WG, De Backer JW, Vinchurkar SC, Verdonck PR, Van Doorn JWD, Nadjmi N, De Backer WA (2013): Change in Upper Airway Geometry Between Upright and Supine Position During Tidal Nasal Breathing. J Aerosol Med 26: 1-7

Visser H, Rödig T, Hermann KP (2001): Dose reduction by direct-digital cephalometric radiography. Angle Orthod 71(3):159-63

Vog1 TJ: Kernspintomographie in der Kopf-Hals-Region. Springer Verlag Berlin 1991

Voßhans J (2005): The anatomical landmarks of bone and teeth with the digital volume tomographie. ZM 95(2): 32-36

Wein B, Drobnitzky M, Klajman S (1990): Magnetresonanztomographie und Sonographie bei der Lautbildung. Fortschr Roentgenstr 153: 408-412

Whittle AT, Marshall I, Mortimore IL, Wraith PK, Sellar RJ, Douglas NJ (1999): Neck soft tissue and fat distribution: comparison between normal men and women by magnetic resonance imaging. Thorax 54 (4): 323-328

Yucel A, Unlu M, Haktanir A, Acar M, Fidan F (2005): Evaluation of the Upper Airway Crosssectional Area Changes in Different Degrees of Severity of Obstructive Sleep Apnea Syndrome: Cephalometric and Dynamic CT Study. Am J Neuroradiol 26: 2624-2629 
Ziegler CM, Woertche R, Brief J (2002): Clinical Indications for digital volume tomography in oral and maxillofacial surgery. Dentomaxillofac Radiol 31: 126-130 


\section{Danksagung}

Als erstes möchte ich mich bei meinem Doktorvater Prof. Dr. med. Dr. med. dent. Wilfried Engelke bedanken. Er hat mir nicht nur die Möglichkeit gegeben, bei ihm die Doktorarbeit zu schreiben, sondern hat auch mit seinem Ideenreichtum und der guten Betreuung zum Gelingen der vorliegenden Arbeit beigetragen.

Auch Daniel Markiewicz gebührt mein Dank für die zahlreichen konstruktiven Gespräche zum Thema. 


\section{Lebenslauf}

Am 23.02.1984 wurde ich als Sohn von Dr. Wolf - Rüdiger Schlick und Heidemarie

Rathmann in der Lutherstadt Wittenberg geboren.

Vom 1990 bis 1994 ging ich auf die Friedrich-Engels-Grundschule in Piesteritz.

Ab 1994 besuchte ich das Lucas-Cranach-Gymnasium in Piesteritz, auf dem ich das Abitur 2003 absolvierte.

Vom August 2003 bis Mai 2004 leistete ich meinen Zivildienst am Paul- Gerhard- Stift auf der Station für Allgemeine und Traumatologische Chirurgie in der Lutherstadt Wittenberg ab.

Ab dem Sommersemester 2005 studierte ich an der Georg- August- Universität in Göttingen Zahnmedizin und arbeitete vom April 2007 bis März 2008 in der Abteilung für Prothetik als Hilfswissenschaftler. 2006 bestand ich das Vorphysikum und 2007 das Physikum.

Am 24.11.2010 schloss ich das Zahnmedizinstudium mit dem Staatsexamen ab. 
Abstract

Schlick, Christoph

Struktur und Dimension des oropharyngealen Luftweges im Digitalen Volumentomographen (DVT)

Fragestellung. Mit der Digitalen Volumentomographie (DVT) wurde 1997 in die Zahn-, Mund- und Kieferheilkunde eine neue Aufnahmetechnik eingeführt, die dieselben Rekonstruktions-möglichkeiten wie die Computertomographie (CT) und Magnetresonanztomographie (MRT) bietet. Auf der Basis vorhandener DVT-Datensätze sollte der Luftweg im Bereich des orofazialen Systems vermessen werden. Die dabei gemessenen Parameter dienten der Erstellung von Normwerten in horizontaler Körperposition mit Schwerpunkt der Ermittlung geschlechtsspezifischer Unterschiede. Des Weiteren sollten die Zusammenhänge anatomischer Faktoren des aufsteigenden Unterkieferastes und der Luftwegsdimension dargestellt werden.

Methodik. Die Bestimmung des oberen Luftweges wurde an 129 Patienten (65 Frauen und 64 Männer), im Alter zwischen 30 und 68 Jahren, vorgenommen, die aufgrund anderer Indikationsstellung mittels des Digitalen Volumentomographen NewTom QR-DVT 9000@ mit einer Röhrenspannung von $110 \mathrm{kV}$ und einem Röhrenstrom zwischen 0,5-10 mA geröntgt wurden. Dabei mussten die anatomischen Strukturen von der Nasallinie bis zum Hyoid abgebildet sein. Die Auswertung wurde mit dem Softwareprogramm NewTom 9000 Dental Version 3.37 durchgeführt. Im Rahmen der Untersuchung wurden unteranderen folgende Parameter bestimmt: die Hartgaumenlänge, die Weichgaumenlänge und Weichgaumenbreite, die Länge des kollapsfähigen Segments, der Abstand von Hyoid zur Nasallinie, die sagittalen Dimension auf Höhe des Inzisialpunktes (MPW), auf Höhe des dorsalsten Punktes der Zunge (TPW) und auf Höhe der Vallecula glossoepiglottica (LPW), sowie die Neigung des Unterkieferastes zur mediosagittalen Ebene (Winkel $\alpha$ und Winkel $\beta$ ). Es wurde eine deskriptive Auswertung durchgeführt. Der Vergleich der metrischen Werte erfolgte mit Hilfe von Korrelationsmatrizen nach Pearson. Die Gegenüberstellung zweier unabhängiger Stichproben (Vergleich Geschlecht mit metrischen Werten) wurde mit dem Mann-Whitney-UTest durchgeführt. Das Signifikanzniveau wurde auf $\mathrm{p}<0,05$ festgelegt.

Resultate: Bei Messungen in der mediosagittalen Ebene wurden signifikante Unterschiede zwischen den Geschlechtern festgestellt: bei der Hartgaumenlänge (Männer: 56,64 mm \pm 3,02, Frauen: 52,72 $\mathrm{mm} \pm 2,82$ ), bei der Weichgaumenlänge (Männer: 41,93 $\mathrm{mm} \pm 5,24$, Frauen: 35,99 mm $\pm 3,61$ ) und Weichgaumenbreite (Männer: 10,83 mm $\pm 1,71$, Frauen: 9,31 $\mathrm{mm} \pm 2,41$ ), die Länge des kollapsfähigen Segments (Männer: 59,52 mm \pm 7,83, Frauen: 
50,38 $\mathrm{mm} \pm 5,27$ ) und beim Abstand von Hyoid zur Nasallinie (Männer: 70,63 mm \pm 6,71, Frauen: 56,97 $\mathrm{mm} \pm 5,42$ ). Auch bei den Luftwegsdimensionen gab es signifikante Unterschiede und zwar in der sagittalen Dimension auf Höhe des Inzisialpunktes (MPW) (Männer: 8,14 mm $\pm 2,75$, Frauen: 9,24 mm $\pm 3,15$ ), auf Höhe des dorsalsten Punktes der Zunge (TPW) (Männer: 11,99 $\mathrm{mm} \pm 3,56$, Frauen: 10,14 $\mathrm{mm} \pm 2,91$ ) und auf Höhe der Vallecula glossoepiglottica(LPW) (Männer: 14,46 mm $\pm 2,61$, Frauen: 11,55 mm $\pm 2,50$ ). Auch bei den Querschnittsflächen konnte signifikante Unterschiede festgestellt werden: auf Höhe des Inzisialpunktes (MPW) (Männer: 123,37 $\mathrm{mm}^{2} \pm 82,52$, Frauen: 140,97 $\mathrm{mm}^{2} \pm$ 64,98), auf Höhe des dorsalsten Punktes der Zunge (TPW) (Männer: 243,63 mm² $\pm 108,31$, Frauen: 197,89 $\left.\mathrm{mm}^{2} \pm 86,54\right)$ und auf Höhe der Vallecula glossoepiglottica(LPW) (Männer: $333,94 \mathrm{~mm}^{2} \pm 125,91$, Frauen: $276,06 \mathrm{~mm}^{2} \pm 94,56$ ). Keine signifikante Unterschiede konnte in den transversalen Durchmessern, beim Luftquerschnitt auf Höhe des Isthmus (Männer: $91,82 \mathrm{~mm}^{2} \pm 57,74$, Frauen: $\left.94,89 \mathrm{~mm}^{2} \pm 51,98\right)$ und bei der Neigung des Unterkieferastes zur mediosagittalen Ebene (Winkel $\alpha$ und Winkel $\beta$ ) festgestellt werden.

Schlussfolgerung. Insgesamt konnte man feststellen, dass es zahlreiche anatomische Unterschiede in Bezug auf den oberen Luftweg zwischen den beiden Geschlechtern gibt. Der hohe Frauenanteil ermöglichte eine höhere Aussagekraft in Bezug auf den Geschlechtervergleich. Die wichtigsten signifikanten Unterschiede waren vor allem Weichgaumenlänge und- breite, die Länge des kollapsfähigen Segments, der Abstand von Hyoid und Nasallinie, Querschnitte und sagittale Ausdehnung des Luftweges auf Höhe der dorsalsten Stelle der Zunge und auf Höhe der Vallecula glossoepiglottica. Hier waren die Ergebnisse bei den Männern deutlich größer. Dagegen war der Luftweg auf Höhe des Inzisialpunktes bei den Frauen größer. Keine Unterschiede konnten im Bereich des Nasopharynx und im Bereich des Isthmus, sowie in den transversalen Dimensionen festgestellt werden.

Auch die Rolle der Körperposition muss bei der Auswertung miteinbezogen werden. Anscheinend hat die Körperposition nur im Bereich hinter dem Velum einen Einfluss auf die Luftwegsdimension. In liegender Position scheinen die Luftwegsquerschnitte deutlich kleiner zu sein als in aufrechter.

Das NewTom QR-DVT 9000@ liefert wertvolle Informationen über die Luftwegsdimensionen in horizontaler Lage. 